ANALYSIS \& PDE

2011

ROBERT JERRARD

DEFECTS IN SEMILINEAR WAVE EQUATIONS AND TIMELIKE

MINIMAL SURFACES IN MINKOWSKI SPACE

Volume $4 \quad$ No. 2 


\title{
DEFECTS IN SEMILINEAR WAVE EQUATIONS AND TIMELIKE MINIMAL SURFACES IN MINKOWSKI SPACE
}

\author{
ROBERT JERRARD
}

\begin{abstract}
We study semilinear wave equations with Ginzburg-Landau-type nonlinearities, multiplied by a factor of $\varepsilon^{-2}$, where $\varepsilon>0$ is a small parameter. We prove that for suitable initial data, the solutions exhibit energy-concentration sets that evolve approximately via the equation for timelike Minkowski minimal surfaces, as long as the minimal surface remains smooth. This gives a proof of the predictions made (on the basis of formal asymptotics and other heuristic arguments) by cosmologists studying cosmic strings and domain walls, as well as by applied mathematicians.
\end{abstract}

\section{Introduction}

In this paper we prove that, if $\Gamma$ is a timelike minimal surface of codimension $k=1$ or 2 in Minkowski space $\mathbb{R}^{1+N}$, smooth in a time interval $(-T, T)$, then, for suitable initial data and $N>k$, the solutions $u: \mathbb{R}^{1+N} \rightarrow \mathbb{R}^{k}$ of the equation

$$
\square u+\frac{1}{\varepsilon^{2}} f(u)=0, \quad 0<\varepsilon \ll 1,
$$

exhibit an energy-concentration set that approximately follows $\Gamma$, at least up to time $T$. Here, the model nonlinearity is $f(u)=\left(|u|^{2}-1\right) u$ in low dimensions; in higher dimensions, we take $f$ to be a qualitatively similar nonlinearity, satisfying growth conditions that leave equation (1-1) globally well-posed; see (1-9) and (1-19) for the precise assumptions.

Our main motivation for this work comes from the very rich mathematical literature on corresponding questions about elliptic and parabolic analogues of (1-1), which have been studied in great detail for the past 30 years or so. In the elliptic case, these past results establish deep connections between energyconcentration sets for the solutions $u: \Omega \subset \mathbb{R}^{N} \rightarrow \mathbb{R}^{k}$ of the equation

$$
-\Delta u+\frac{1}{\varepsilon^{2}} f(u)=0, \quad 0<\varepsilon \ll 1,
$$

and (Euclidean) minimal surfaces of codimension $k$ in $\Omega$. Similarly, the parabolic equation

$$
u_{t}-\Delta u+\frac{1}{\varepsilon^{2}} f(u)=0, \quad 0<\varepsilon \ll 1, \quad u:(0, T) \times \mathbb{R}^{N} \rightarrow \mathbb{R}^{k},
$$

The author was partially supported by the National Science and Engineering Research Council of Canada under operating Grant 261955.

MSC2000: primary 35B40, 35L70, 53C44; secondary 85A40.

Keywords: Minkowski minimal surface, semilinear wave equation, topological defects, defect dynamics. 
is related to the geometric evolution problem of codimension- $k$ motion by mean curvature. Our results address the natural question of whether any parallel results hold, relating the semilinear wave equation (1-1) to the timelike Minkowski minimal surface problem, which is a geometric wave equation.

It turns out that this question is also relevant to the description of cosmological domain walls $(k=1)$ and strings $(k=2)$; see [Kibble 1976] for a seminal early paper, and [Vilenkin and Shellard 1994] for an in-depth survey of a large body of work on related questions. The problems we study have also been addressed in the applied math literature by [Neu 1990], with some generalizations considered by [Rotstein and Nepomnyashchy 2000]. We will not say any more about any of these applications in this paper, except to note that our main results can be described as giving a rigorous derivation, in the relatively simple and physically unrealistic setting of a scalar particle described by equation (1-1), of the laws of motion for cosmic strings and domain walls, deduced formally by cosmologists over 30 years ago.

1.1. Mathematical background. We first review results about the elliptic and parabolic equations (1-2) and (1-3). Throughout this discussion, we consider the model nonlinearity $f(u)=\left(|u|^{2}-1\right) u$.

In the elliptic case, and when $k=1$ (so that equation (1-2) is scalar), the general heuristic principle (underlying essentially every work we know of) is that

where $q: \mathbb{R} \rightarrow \mathbb{R}$ solves

$$
u \approx q\left(\frac{d}{\varepsilon}\right),
$$

$$
-q^{\prime \prime}+f(q)=0, \quad q(0)=0 \quad \text { and } \quad q(x) \rightarrow \pm 1 \text { as } x \rightarrow \pm \infty,
$$

and $d: \Omega \rightarrow \mathbb{R}$ is the signed-distance function to a minimal hypersurface $\Gamma \subset \Omega$, so that $d$ is characterized near $\Gamma$ by the properties

and $\Gamma$ satisfies

$$
d=0 \text { on } \Gamma, \quad|\nabla d|^{2}=1 \text { near } \Gamma,
$$

$$
\text { (Euclidean) mean curvature }=0 .
$$

There are a vast number of results establishing various forms of these assertions. Roughly speaking, these fall into two families. The first (see for example [Modica 1987] or [Hutchinson and Tonegawa 2000]) employ variational and measure-theoretic methods, together with elliptic estimates, to characterize the limiting behavior of sequences of solutions as $\varepsilon \rightarrow 0$. These proofs generally establish some form of what is called "equipartition of energy", which can be viewed as a weak form of the description (1-4). The second family of proofs (see for example [Pacard and Ritoré 2003]) employ the Liapunov-Schmidt reduction and related arguments, relying ultimately on the implicit function theorem and control of the spectrum of some linearized operator. These arguments yield existence results that give very precise descriptions, in the spirit of (1-4), of the solutions that are constructed.

In the $k=1$ scalar case of the parabolic equation (1-3), more or less the same heuristic (1-4), (1-5) holds, except that now $d$ is a function of $t$ and $x$, and, for every $t, d(t, \cdot)$ is the signed-distance function from a hypersurface $\Gamma_{t}$, so that

$$
d(t, \cdot)=0 \text { on } \Gamma_{t} \quad \text { and } \quad\left|\nabla_{x} d(t, \cdot)\right|^{2}=1 \text { near } \Gamma_{t},
$$

with $\Gamma:=\bigcup_{t>0}\{t\} \times \Gamma_{t}$ inside $(0, T) \times \mathbb{R}^{N}$, satisfying

$$
\text { velocity }=\text { mean curvature. }
$$


Different versions of this result have been established by a variety of proofs, including linearization techniques (see [de Mottoni and Schatzman 1995]), which establish a strong form of (1-4) but are valid only locally in $t$; maximum principle arguments, which ultimately rely on an ansatz based on (1-4) to build sub- and super-solutions (see [Chen 1992; Evans et al. 1992]), or which employ a change of variables motivated by (1-4) and techniques for weak passage to limits [Barles et al. 1993]; and measuretheoretic methods combined with parabolic estimates (as in [Ilmanen 1993]), in which (1-4) appears in the weak form of assertions about equipartition of energy. The maximum principle and measure-theoretic arguments give weaker descriptions that are, however, valid globally in $t$, with (1-8) understood in a weak sense.

In the vector-valued $k=2$ case, for both the elliptic (1-2) and parabolic (1-3) systems, we do not know of any characterization as precise as (1-4); obstacles to such results include the difficulty of describing rotational degrees of freedom, and the related poor behavior of the spectrum of certain linearized operators. However, there are a number of results showing, in various degrees of generality for solutions of equation (1-2) (including, among others, [Lin and Rivière 1999; Bethuel et al. 2001; Alberti et al. 2005]) and of equation (1-3) (see [Ambrosio and Soner 1997; Lin and Rivière 2001; Bethuel et al. 2006], for example) with suitable energy bounds, that energy concentrates around a codimension-2 submanifold $\Gamma$ satisfying (1-7) and (1-8), respectively. These results generally employ elliptic or parabolic estimates, some of which are extremely delicate, in combination with measure-theoretic arguments, and they provide information, customarily phrased in the language of varifold convergence, about the precise way in which energy concentrates around the codimension- 2 surface $\Gamma$.

All results about (1-2) and (1-3) rely very heavily on tools that are not available for hyperbolic equations, such as maximum principles (in the scalar case) and elliptic or parabolic regularity. Thus, they do not give much indication of how to proceed for the nonlinear wave equation (1-1). We know of only two partial exceptions to this rule. First, there is no abstract reason that linearization arguments should be impossible in the hyperbolic setting; they appear however to be hard to carry through. Second, a number of papers, starting with [Bronsard and Kohn 1991], study (1-3) using weighted energy estimates. In particular, we mention an argument presented by Soner in a 1995 lecture series [1998] for the scalar parabolic equation (1-3), and developed in [Jerrard and Soner 1999; Lin 1998] for parabolic systems. This argument relies on a rather straightforward but remarkable computation of $\frac{d}{d t} \int_{\mathbb{R}^{N}} \zeta e_{\varepsilon}(u) d x$, where $e_{\varepsilon}(u)$ is a natural energy density associated with a solution $u$ of (1-3), and $\zeta$ is a smooth function such that $\zeta(t, x)=\frac{1}{2} \operatorname{dist}\left(x, \Gamma_{t}\right)^{2}$ near $\Gamma_{t}$, where the latter solves (1-8). This calculation certainly uses the parabolic character of (1-3), but it is not clear if it uses it in an essential way. Indeed, our main proofs originated as an attempt to develop an analogue of this argument in the hyperbolic setting.

Much less work has been done on the hyperbolic equation (1-1) than on its elliptic and parabolic counterparts. The few papers of which we are aware mostly study situations rather different from those we consider here, including

- works [Jerrard 1999; Lin 1999] that characterize the behavior of solutions of (1-1) in the limit $\varepsilon \rightarrow 0$ in the case $N=k=2$, for the model nonlinearity $f(u)=\left(|u|^{2}-1\right) u$.

- [Gustafson and Sigal 2006], on the Maxwell-Higgs model, in which (1-1), with the model nonlinearity $f(u)=\left(|u|^{2}-1\right) u$, is coupled to an electromagnetic field, when $N=k=2$ and $0<\varepsilon \ll 1$. 
- [Stuart 2004a], studying an equation of the form (1-1) on a Lorentzian manifold and with a focusing nonlinearity, for $0<\varepsilon \ll 1$; see also [Stuart 2004b].

In all these papers, energy concentrates around points (known as "vortices" or "quasiparticles", depending on the situation), and these points evolve according to an ODE. These results are valid only as long as the points remain separated from each other. The fact that points are geometrically very simple objects makes the analysis easier, in some ways, than in the problems we consider here, where the same role is now played by submanifolds of dimension $n \geq 1$. An additional significant, simplifying factor in all the papers cited above (except those of Stuart) is that they study a scaling in which vortices move at subrelativistic velocities, that is, velocities that tend to 0 as $\varepsilon \rightarrow 0$.

It is also worth mentioning the work of Cuccagna [2008] that studies (1-1) in $\mathbb{R}^{1+3}$ with $\varepsilon=1$, and establishes scattering for initial data $\left.\left(u, u_{t}\right)\right|_{t=0}$, which is a small, very smooth perturbation of $\left(q\left(x^{3}\right), 0\right)$. This can be seen as an analogue for (1-1) of results [Lindblad 2004; Brendle 2002] that establish scattering for solutions of the timelike Minkowski minimal surface problem, with initial data that is a small perturbation of a motionless hyperplane.

As far as we know, the only work of rigorous mathematics that addresses exactly the questions we consider here is a recent preprint of Bellettini, Novaga, and Orlandi [2008]. Its main result identifies some conditions that, if they could be verified, would suffice to imply that a varifold, obtained from a sequence of solutions $\left(u_{\varepsilon}\right)$ of (1-1) satisfying natural energy bounds, is stationary with respect to the Minkowski inner product structure. These conditions include lower density bounds, as well as, roughly speaking, some quite strong constraints on the limiting tangent space. As discussed in Remark 1.6, the results we obtain here are stronger than those projected in [Bellettini et al. 2008].

1.2. New results. In many ways, our results follow the pattern described previously. In the case $k=1$ of a scalar equation, as in the earlier work on the elliptic and parabolic problems, we obtain, for suitable initial data, a description of solutions of (1-1) parallel to (1-4), (1-5), (1-6), (1-7) with, in the last two identities, the Euclidean metric replaced by the Minkowski metric. And in the case $k=2$, we prove that, for solutions of (1-1) with suitable initial data, energy concentrates around a codimension-2 surface $\Gamma$ that satisfies (1-7), again with the Euclidean metric replaced by the Minkowski metric. We also give a precise description of the way in which this concentration occurs; in fact, we obtain this description for the case $k=1$ as well.

The strongest results (for example [Bethuel et al. 2006]) on the parabolic equation (1-3) hold globally for $t>0$, and they assume only natural energy bounds on the initial data. Our results, by contrast, are valid only locally in $t$ - that is, as long as the surface $\Gamma$ remains smooth — and require rather special initial data. We note, however, that results like those we obtain are almost certainly not true globally in $t$ or for general initial data.

In all our results, we take the timelike minimal surface $\Gamma$ to have the topology of $(-T, T) \times \mathbb{T}^{n}$, where $n=N-k$. When $k=2$, this covers the important example of a closed string in $\mathbb{R}^{3}$. In fact, we view the global topology of $\Gamma$ as relatively unimportant, since our results are in some sense local, and since both the semilinear wave equation (1-1) and the timelike minimal surface equation enjoy finite propagation speed. In any case, our methods should extend to $\Gamma \cong(-T, T) \times M$ for more general $M$.

The quite general results in [Milbredt 2008] imply, in particular, the local existence of smooth timelike minimal surfaces $\Gamma$, given smooth data at $t=0$. 
In the scalar case, we assume that the nonlinearity $f$ in (1-1) has the form $f=F^{\prime}$, where $F: \mathbb{R} \rightarrow \mathbb{R}$ is a smooth function such that

$$
F( \pm 1)=0 \quad \text { and } \quad c(1-|s|)^{2} \leq F(s) .
$$

We also assume that $f$ grows sufficiently slowly so that (1-1) is globally well posed in $\dot{H}^{1} \times L^{2}$. If $N \leq 4$, we may take $f(u)=\left(u^{2}-1\right) u$.

In the statement of our results, we use the notation

and

$$
e_{\varepsilon}(u):=\frac{1}{2}\left(u_{t}^{2}+|\nabla u|^{2}\right)+\frac{1}{\varepsilon^{2}} F(u)
$$

$$
\kappa_{1}:=\int_{-1}^{1} \sqrt{2 F(s)} d s
$$

One can think of $\kappa_{1}$ as a constant, related to the surface tension of an interface. In the scalar case, our main results can be summarized as:

Theorem 1. Let $\Gamma \subset(-T, T) \times \mathbb{R}^{N}$ be a smooth timelike minimal hypersurface. Let $\Gamma_{t}:=\Gamma \cap\left(\{t\} \times \mathbb{R}^{N}\right)$ and assume that, for every $t \in(-T, T), \Gamma_{t}$ is diffeomorphic to the torus $\mathbb{T}^{n}$, for $n=N-1$.

Given $T_{0}<T$, there exists a neighborhood $\mathcal{N}$ of $\Gamma$ in $\left(-T_{0}, T_{0}\right) \times \mathbb{R}^{N}$ in which there exists a smooth solution $d: \mathcal{N} \rightarrow \mathbb{R}$ of the problem

$$
d=0 \text { on } \Gamma, \quad-d_{t}^{2}+|\nabla d|^{2}=1 \text { near } \Gamma .
$$

(In other words, $d$ is the signed Minkowski distance to $\Gamma$; compare with (1-6).) Moreover, there exists a solution $u$ of (1-1) (with $f$ as described above) such that, for any $T_{0}<T$,

where $q$ solves (1-5) and

$$
\left\|u-q\left(\frac{d}{\varepsilon}\right)\right\|_{L^{2}(\mathcal{N})} \leq C \sqrt{\varepsilon}
$$

$$
\int_{\mathcal{N}} d^{2} e_{\varepsilon}(u) d t d x+\int_{\left[\left(-T_{0}, T_{0}\right) \times \mathbb{R}^{N}\right] \backslash \mathcal{N}} e_{\varepsilon}(u) d t d x \leq C \varepsilon .
$$

In addition, if $\mathscr{T}_{\varepsilon(u)}=\left(\mathscr{T}_{\varepsilon, \beta}^{\alpha}(u)\right)_{\alpha, \beta=0}^{N}$ and $\mathscr{T}(\Gamma)=\left(\mathscr{T}_{\beta}^{\alpha}(\Gamma)\right)_{\alpha, \beta=0}^{N}$ denote the energy-momentum tensors for $u$ and $\Gamma$ (defined in (2-8) and (2-9), respectively), then

$$
\left\|\frac{\varepsilon}{\kappa_{1}} \mathscr{T}_{\varepsilon}(u)-\mathscr{T}(\Gamma)\right\|_{W^{-1,1}\left(\left(-T_{0}, T_{0}\right) \times \mathbb{R}^{N}\right)} \leq C \varepsilon .
$$

In all these conclusions, $C=C\left(T_{0}, \Gamma\right)$ is independent of $\varepsilon$.

Remark 1.1. The definitions imply that $\mathscr{T}_{\varepsilon, 0}^{0}(u)=e_{\varepsilon}(u)$, and that $\mathscr{T}_{0}^{0}(\Gamma)$ is a measure supported on $\Gamma$ and defined by

$$
\int f(t, x) d \mathscr{T}_{0}^{0}=\int_{-T}^{T} \int_{\Gamma_{t}} f(t, x)\left(1-V^{2}\right)^{-1 / 2} \mathcal{H}^{n}(d x) d t
$$

where $V(t, x)$ denotes the (Euclidean) normal velocity of $\Gamma$ at a point $(t, x) \in \Gamma$. We can denote this measure by $\left(1-V^{2}\right)^{-1 / 2}\left(\mathscr{H}^{n}\left\llcorner\Gamma_{t}\right) \otimes d t\right.$. The conclusion (1-15) thus implies, in particular, that

$$
\| \frac{\varepsilon}{\kappa_{1}} e_{\varepsilon}(u)-\left(1-V^{2}\right)^{-1 / 2}\left(\mathscr{H}^{n}\left\llcorner\Gamma_{t}\right) \otimes d t \|_{W^{-1,1}\left(\left(-T_{0}, T_{0}\right) \times \mathbb{R}^{N}\right)} \leq C \varepsilon .\right.
$$


A parallel remark holds for conclusion (1-21) of Theorem 2 below.

Remark 1.2. Our arguments show that a solution $u$ of (1-1) satisfies (1-13), (1-14) and (1-15) if, for example,

$$
u(0, x)=q\left(\frac{d(0, x)}{\varepsilon}\right) \quad \text { and } \quad u_{t}(0, x)=\frac{1}{\varepsilon} q^{\prime}\left(\frac{d(0, x)}{\varepsilon}\right) d_{t}(0, x)
$$

in a neighborhood $\mathcal{N}_{0}$ of $\Gamma_{0}$, and if

$$
\int_{\{0\} \times\left(\mathbb{R}^{N} \backslash \mathcal{N}_{0}\right)} e_{\varepsilon}(u) d x \leq \varepsilon .
$$

For details, see Lemma 9 and Theorem 22.

In the vector case, we can again take $f(u)=\left(|u|^{2}-1\right) u$ if $N \leq 4$, or, in other words, $f=\nabla_{u} F$, for $F(u)=\frac{1}{4}\left(|u|^{2}-1\right)^{2}$. More generally, we require from $f$ only that the equation (1-1) be globally well posed in $\dot{H}^{1} \times L^{2}$, and that $f=\nabla_{u} F$ where

$$
c(1-|u|)^{2} \leq F(u) \leq C(1-|u|)^{2} \text { for }|u| \leq 2 \quad \text { and } \quad F(u) \geq c>0 \text { for }|u| \geq 2 .
$$

We summarize our results in the $k=2$ vector case in:

Theorem 2. Let $\Gamma \subset(-T, T) \times \mathbb{R}^{N}$ be a smooth timelike minimal surface of codimension $k=2$. Let $\Gamma_{t}:=\Gamma \cap\left(\{t\} \times \mathbb{R}^{N}\right)$ and assume that, for every $t \in(-T, T), \Gamma_{t}$ is diffeomorphic to the torus $\mathbb{T}^{n}$, for $n=N-2 \geq 1$.

When $k=2$, there exists a solution for (1-1) such that, for any $T_{0}<T$, there is a constant $C$ such that

$$
\int_{\left(-T_{0}, T_{0}\right) \times \mathbb{R}^{N}} \tilde{d}^{2} e_{\varepsilon}(u) d t d x \leq C,
$$

where $\tilde{d}(t, x)=\min \{1, \operatorname{dist}((t, x), \Gamma)\}$ and

$$
\left\|\frac{1}{\pi|\ln \varepsilon|} \mathscr{T}_{\varepsilon}(u)-\mathscr{T}(\Gamma)\right\|_{W^{-1,1}\left(\left(-T_{0}, T_{0}\right) \times \mathbb{R}^{N}\right)} \leq C|\ln \varepsilon|^{-1 / 2}
$$

where $\mathscr{T}_{\varepsilon}(u)$ and $\mathscr{T}(\Gamma)$ denote the energy-momentum tensors for $u$ and $\Gamma$ defined in (2-8) and (2-9), respectively. In all these conclusions, $C=C\left(T_{0}, \Gamma\right)$ is independent of $\varepsilon$.

Remark 1.3. In Lemma 9 we give an explicit construction of initial data for which the conclusions of the theorem hold.

Remark 1.4. The proof shows that the solutions $u$ from Theorem 2 have a defect near $\Gamma$; see (6-5) for a precise, if opaque, version of this assertion.

Remark 1.5. In both the above theorems, the constants $C$ in the conclusions are at least exponential in $T_{0}$. That is, our proofs yield constants of the form $C=a e^{b T_{0}}$, where $a, b$ themselves depend on $\Gamma$ and $T_{0}$, and may blow up as $T_{0} \nearrow T$.

Remark 1.6. Our results imply in particular that, if we fix $\Gamma$ as in either of the theorems above, there exists a sequence $\left(u_{\varepsilon}\right)$ of solutions of (1-1) such that the energy-momentum tensors $\delta_{\varepsilon} \mathscr{T}_{\varepsilon}\left(u_{\varepsilon}\right)$ converge weakly, as measures in $(-T, T) \times \mathbb{R}^{N}$, to $\mathscr{T}(\Gamma)$, if the scaling factor $\delta_{\varepsilon}=\delta_{\varepsilon}(k)$ is chosen correctly. This fact can be seen as a form of varifold convergence, analogous to results proved in [Ilmanen 1993; 
Ambrosio and Soner 1997; Bethuel et al. 2001; 2006] for elliptic and parabolic equations, and discussed in the hyperbolic case in [Bellettini et al. 2008].

However, by providing quantitative estimates of $\left\|\delta_{\varepsilon} \mathcal{T}_{\varepsilon}(u)-\mathscr{T}(\Gamma)\right\|_{W^{-1,1}}$, our results are sharper than simple convergence results. This sharpening is significant, because convergence results, strictly analogous to known results in the elliptic or parabolic cases, can fail in the hyperbolic setting. That is, in our setting (but not for elliptic or parabolic problems) there exist sequences of solutions $\left(u_{\varepsilon}\right)$ such that $\delta_{\varepsilon} \mathscr{T}_{\varepsilon}\left(u_{\varepsilon}\right)$ converges to a measure-valued tensor $\mathscr{T}$ supported on a codimension- $k$ set, but such that $\mathscr{T}$ is not the energy-momentum tensor for any timelike minimal surface $\Gamma$; in other words, $\mathscr{T}$ is not weakly stationary; see Section 1.4 for explicit examples.

Remark 1.7. If we fix $\Gamma$ and consider an associated sequence $\left(u_{\varepsilon}\right)$ of solutions as found in Theorem 2 , with $\varepsilon \rightarrow 0$, the uniform energy bounds (1-20) away from $\Gamma$, combined with a classical argument of [Shatah 1988], imply that, after passing to a subsequence, $u_{\varepsilon}$ converges weakly in $H_{l o c}^{1}([(-T, T) \times$ $\left.\left.\mathbb{R}^{N}\right] \backslash \Gamma\right)$ to a wave map into $S^{1}$.

Remark 1.8. In both theorems, we ultimately rely on energy estimates in a frame that moves with $\Gamma$. These estimates (summarized in Theorem 22) assert more or less that energy remains concentrated around $\Gamma$ on the same scale for $0<t<T$, as it is at $t=0$. The hypotheses for Theorem 22 are:

- small energy away from $\Gamma_{0}-$ see (2-31);

- a defect near $\Gamma_{0}-$ see (2-36);

- small energy, given the presence of the defect, near $\Gamma_{0}$, in a frame that moves with $\Gamma$ - see (2-34) and (2-35).

Theorems 1 and 2 follow from the special case of Theorem 22 in which the energy is, roughly speaking, as concentrated as possible around $\Gamma_{0}$. The fact that our results for $k=1$ are considerably stronger than for $k=2$ stems ultimately from the fact that, when $k=1$, for initial data that is nearly energetically optimal (essentially, (1-17) and (1-18) or suitably small perturbations thereof) the energy is very sharply concentrated around $\Gamma_{0}$, whereas when $k=2$, for the model initial data, energy is quite spread out. A more precise expression of this fact appears in (1-33).

1.3. About the proofs. A main issue in the analysis of (1-1) is to establish some kind of stability property of the moving defect; that is, the interface $(k=1)$ or "string" $(k=2)$. The relativistic invariance of the equation suggests that a defect should acquire extra energy when it accelerates (and this is confirmed by our results; see, for example, (1-16)), so we must rule out this extra energy as a potential source of instability. Our analysis starts from the observation that, for a solution that behaves as predicted in the formal arguments of [Vilenkin and Shellard 1994; Neu 1990; Rotstein and Nepomnyashchy 2000] and others, a moving defect will always appear to be energetically optimal in the frame of reference of an observer who is moving with the defect.

1.3.1. Change of variables. Motivated by this, we begin by rewriting the equation in a frame that follows the timelike minimal surface $\Gamma$, where the defect is expected to remain. In these variables, our task is to show that the solution is approximately constant, and we expect the defect to have some optimality property that we can exploit. 
To define the change of variables, we start with a map $H$ defined on $(-T, T) \times \mathbb{T}^{n}$ and parametrizing $\Gamma \subset(-T, T) \times \mathbb{R}^{N}$, and we extend $H$ to a diffeomorphism $\psi$ between, essentially, a neighborhood in $(-T, T) \times \mathbb{T}^{n} \times \mathbb{R}^{k}$ of $(-T, T) \times \mathbb{T}^{n}$ and a neighborhood of $\Gamma$ in $\mathbb{R}^{1+N}$. We write $\psi$ as a function of variables $y=\left(y^{0}, \ldots, y^{N}\right)=\left(y^{\tau}, y^{\nu}\right)$, where $y^{\tau}=\left(y^{0}, \ldots, y^{n}\right)$ are variables tangent to $\Gamma$, and $y^{v}=\left(y^{n+1}, \ldots, y^{N}\right)$ correspond to directions normal to $\Gamma$. We always arrange that $y^{0}$ is a timelike coordinate, and that all other coordinates are spacelike.

We will also write, for example, $D_{\tau}=\left(\partial_{y^{0}}, \ldots, \partial_{y^{n}}\right)$ and $\nabla_{v}=\left(\partial_{y^{n+1}}, \ldots, \partial_{y^{N}}\right)$. We generally write $D$ for a space-time gradient, and $\nabla$ for a gradient involving space-like variables only.

We then define $v=u \circ \psi$ on the domain of $\psi$. We find it convenient to write the equation satisfied by $v$ (that is, equation (1-1) expressed in terms of the $y$ variables) in the form

$$
-\partial_{y^{\alpha}}\left(g^{\alpha \beta} \partial_{y^{\beta}} v\right)-b \cdot D v+\frac{1}{\varepsilon^{2}} f(v)=0 \quad \text { and } \quad b^{\beta}:=\frac{\partial_{y^{\alpha}} \sqrt{-g}}{\sqrt{-g}} g^{\alpha \beta} .
$$

Here, $G=\left(g_{\alpha \beta}\right)$ is the expression in the $y$ coordinates of the Minkowski metric, $\left(g^{\alpha \beta}\right)=\left(g_{\alpha \beta}\right)^{-1}, g=$ $\operatorname{det}\left(g_{\alpha \beta}\right)$, and we implicitly sum over repeated indices. Equation (1-22) enjoys certain useful properties, which are summarized in Proposition 4. Some of these follow from the specific form we chose for the map $\psi$. The fact that $\Gamma$ is a timelike minimal surface implies a key property of the coefficient $b$ of the first-order term:

$$
\left|b^{\nu}\right| \leq C\left|y^{\nu}\right| \quad \text { at } y=\left(y^{\tau}, y^{\nu}\right), \quad \text { for } b^{\nu}:=\left(b^{n+1}, \ldots, b^{N}\right) .
$$

We emphasize that the verification of (1-23) is the only place in our analysis where we explicitly invoke the fact that $\Gamma$ is a minimal surface.

1.3.2. Energy estimates. We now focus on $v$ solving (1-22) on, say, $\left(-T_{1}, T_{1}\right) \times \mathbb{T}^{n} \times B_{v}\left(\rho_{0}\right)$ for some $T_{1}<T$ and $\rho_{0}>0$, where $B_{v}\left(\rho_{0}\right):=\left\{y^{v} \in \mathbb{R}_{v}^{k}:\left|y^{v}\right|<\rho_{0}\right\}$. We will use the notation

$$
e_{\varepsilon, v}(v):=\frac{1}{2}\left|\nabla_{\nu} v\right|^{2}+\frac{1}{\varepsilon^{2}} F(v)
$$

We introduce a scaling factor $\delta_{\varepsilon}=\delta_{\varepsilon}(k)$ (see (2-1)), chosen so that, heuristically,

$$
\delta_{\varepsilon} \int_{\left\{y^{v} \in \mathbb{R}_{v}^{k}:\left|y^{v}\right| \leq \rho_{1}\right\}} e_{\varepsilon, v}(v)\left(y^{\tau}, \cdot\right) d y^{v} \geq 1-o_{\varepsilon}(1) \quad \text { if } v\left(y^{\tau}, \cdot\right) \text { has a defect near } y^{v}=0,
$$

for every fixed $\rho_{1}$; this is made precise later. One of our goals is to show that, if

$$
\zeta_{3}(s):=\delta_{\varepsilon} \int_{\mathbb{T}^{n} \times W_{v}(s)}\left|D_{\tau} v\right|^{2}+\left.\left|y^{v}\right|^{2} e_{\varepsilon, v}(v) d y^{1} \cdots d y^{N}\right|_{y^{0}=s}
$$

is small when $s=0$, say, then it remains small for a range of positive $s$. Here, $W_{v}(s)$ is a neighborhood of the origin in $\mathbb{R}_{v}^{k}$ that may depend on the parameter $s$, but will always contain a ball of fixed radius $\rho$. The smallness of $\zeta_{3}$ is consistent with $v$ having a large amount of energy, as long as it involves mostly the normal energy $e_{\varepsilon, v}(v)$ and is concentrated very near the codimension- $k$ surface $\left\{y^{v}=0\right\}$.

Our strategy is to define some quantity $\zeta_{1}(s)$ such that

$$
\zeta_{1}^{\prime}(s) \leq C \zeta_{3}(s)
$$


and such that, under suitable additional assumptions,

$$
\zeta_{1}(s) \geq c \zeta_{3}(s)-o_{\varepsilon}(1)
$$

A main task will then be to show that these additional assumptions are preserved by equation (1-22). If we can do this, we can easily use Grönwall's inequality to control the growth of $\zeta_{3}$.

For the verification of (1-26), we define the approximately ${ }^{1}$ conserved energy density

$$
e_{\varepsilon}(v)=\frac{1}{2} a^{\alpha \beta} v_{y^{\alpha}} v_{y^{\beta}}+\frac{1}{\varepsilon^{2}} F(v)
$$

where $a^{\alpha \beta}$ is a positive-definite matrix related to $g^{\alpha \beta}$; see (2-16). (When we want to avoid any possibility of confusion, we will write $e_{\varepsilon}(v ; G)$ for the above quantity, and $e_{\varepsilon}(u ; \eta)$ for the energy defined in (1-10), with $\eta$ denoting the expression in the original coordinates of the Minkowski metric.) We further define

$$
\zeta_{1}(s):=\left.\delta_{\varepsilon} \int_{\mathbb{T}^{n} \times W_{v}(s)}\left(1+\kappa_{2}\left|y^{v}\right|^{2}\right) e_{\varepsilon}(v) d y^{1} \cdots d y^{N}\right|_{y_{0}=s}-1,
$$

where $\kappa_{2}$ is a constant to be selected in a moment. (It will turn out later that we can take $\kappa_{2}=1$ in the scalar case.) We hope to show that $\zeta_{1}$ satisfies the properties (1-26) and (1-27) above.

Indeed, as long as the sets $W_{v}(s)$ are chosen to shrink rapidly enough, we will show in Section 3 that the verification of (1-26) follows quite easily from the differential inequality

$$
\frac{\partial}{\partial y^{0}} e_{\varepsilon}(v) \leq \sum_{i=1}^{N} \frac{\partial}{\partial y^{i}} \varphi^{i}+C\left(\left|D_{\tau} v\right|^{2}+\left|y^{v}\right|^{2}\left|\nabla_{\nu} v\right|^{2}\right)
$$

for some vector $\varphi=\left(\varphi^{1}, \ldots, \varphi^{N}\right)$. The differential inequality (1-29), in turn, follows easily from (1-22); see Lemma 6. The key point in (1-29) is the factor $\left|y^{v}\right|^{2}$, which follows from (1-23) and, hence, from the fact that $\Gamma$ is a minimal surface.

To check (1-27), we first note that some of the good properties of (1-22) alluded to above imply that if $\kappa_{2}$ is chosen in a suitable way (see (2-23)), then

$$
\left(1+\kappa_{2}\left|y^{v}\right|^{2}\right) e_{\varepsilon}(v) \geq c\left|D_{\tau} v\right|^{2}+\left(1+\left|y^{v}\right|^{2}\right) e_{\varepsilon, v}(v) .
$$

With this choice of $\kappa_{2}$,

$$
\zeta_{1}(s) \geq c \zeta_{3}(s)+\left.\int_{\mathbb{T}^{n}}\left(\delta_{\varepsilon} \int_{W_{v}(s)} e_{\varepsilon, v}(v) d y^{v}-1\right) d y^{1} \cdots d y^{n}\right|_{y_{0}=s} .
$$

Thus, in view of the choice (1-25) of $\delta_{\varepsilon}$, we can deduce (1-27) as long as we can check that $v(s, \cdot)$ has a defect confined near $\left\{\left(y^{1}, \ldots, y^{N}\right) \in \mathbb{T}^{n} \times \mathbb{R}_{v}^{k}: y^{v}=0\right\}$. (This is the additional assumption mentioned before equation (1-27).)

\footnotetext{
${ }^{1}$ The exact law expressing conservation of energy for (1-1) can, of course, be transposed to the $y$ coordinates. As far as we know, this is not useful for our problem, since it does not distinguish any good property of equation (1-22) resulting from the fact that the change of variables is built around a parametrization of a minimal surface.
} 
1.3.3. A certain stability property. We therefore introduce a "defect confinement functional"

$$
\text { D }: H^{1}\left(\mathbb{T}^{n} \times B_{v}\left(\rho_{0}\right)\right) \rightarrow \mathbb{R}
$$

that is designed to have two properties. (This functional takes quite different forms in the two cases $k=1$ and $k=2$ that we consider; see (3-1) and (5-1).) First, we require that

$$
\mathscr{D}(v(s, \cdot)) \text { small } \Rightarrow \text { "defect is confined" } \Rightarrow \text { lower energy bounds } \Rightarrow(1-27) \text { holds. }
$$

This sort of argument will eventually lead to an inequality of the simple form

$$
\zeta_{3}(s) \leq C\left[\zeta_{1}(s)+\zeta_{2}(s)\right]+o_{\varepsilon}(1)
$$

where

$$
\zeta_{2}(s)=\mathscr{D}(v(s))
$$

Second, we need $\mathscr{D}$ to be such that

$$
\text { changes in } \zeta_{2}(s) \text { can be controlled by } \zeta_{3}(s) \text {. }
$$

Concrete versions of (1-30) and (1-32) are established in Section 3 for $k=1$, and Section 5 for $k=2$. Heuristically, (1-32) should hold because, if the defect strays away from $y^{v}=0$, then it should carry with it concentrations of energy that can be detected by $\zeta_{3}$. In the case $k=1,(1-32)$ will take the simple form $\zeta_{2}(s) \leq 2 \zeta_{2}(0)+C \int_{0}^{s} \zeta_{3}(\sigma) d \sigma$. The corresponding estimate for $k=2$ is similar but slightly more complicated. In both cases, however, by combining (1-31) and a specific concrete version of (1-32) with (1-26), we obtain control over $\zeta_{i}(s)$ for $i=1,2,3$. This gives us a good deal of information about the behavior of $v$, from which all of our main conclusions are ultimately deduced.

One can view (1-31) and (1-32) as a weak stability property of states $w$ for which $\mathscr{D}(w)$ is small and for which the inequality in (1-31) is almost saturated.

The difference in the strength of our conclusions in the cases $k=1$ and $k=2$, discussed in Remark 1.8, stems from the fact that, for optimal initial data,

$$
\text { for } i=1,2,3, \quad \zeta_{i}(0) \approx \begin{cases}\varepsilon^{2} & \text { when } k=1, \\ |\ln \varepsilon|^{-1} & \text { for } k=2\end{cases}
$$

see Lemma 9. This reflects sharper energy concentration around $\left\{y^{v}=0\right\}$ in the case $k=1$.

1.3.4. Some other issues. The change of variables that we employ is defined only in a neighborhood of $\Gamma$. We must therefore combine estimates of $v$ near $\Gamma$ with estimates of $u$ away from $\Gamma$, and then iterate. We verify in Section 6 that this can be done in such a way as to genuinely yield estimates valid up to $\left(-T_{0}, T_{0}\right) \times \mathbb{R}^{N}$ for arbitrary $T_{0}<T$.

Spacelike hypersurfaces of the form $\left\{y^{0}=\right.$ constant $\}$ play a distinguished role in our argument, as it is along these surfaces that the defect structure is nearly energetically optimal for the solutions $v$ that we consider. This near-optimality is manifested, for example, in the fact that inequality (1-31) is nearly saturated. In general, our change of variables $\psi^{-1}$ maps the hypersurface $\left\{(t, x) \in \mathbb{R}^{1+N}: t=0\right\}$ (on which we assume the data for the solution $u$ of (1-1) is given) onto a hypersurface that is smooth and spacelike, but otherwise can be quite arbitrary. So, a certain amount of work is needed to obtain control of $v$ on a suitable portion of some hypersurface $\left\{y^{0}=\right.$ constant $\}$. This is done in Sections 4 and 5.3, and involves mainly technical adjustments to our basic energy estimates as outlined above. This means that 
we carry out our main energy estimates twice, once in a simpler form that can easily be iterated, and once to deal with complications caused by the geometry of the initial hypersurface in the transformed variables. This, and the similarity between the cases $k=1$ and $k=2$, leads to a certain amount of redundancy which, however, enables us to present out argument first in a relatively simple setting, in Section 3; we believe this makes the main ideas easier to grasp.

The technical work of Section 4 could be avoided if we insisted on prescribing data only on spacelike hypersurfaces that have the form $\left\{y^{0}=\right.$ constant $\}$ near $\Gamma_{0}$, but we feel that this would be unnecessarily restrictive.

Finally, we extract all the conclusions of the main theorems from control over quantities such as $\zeta_{1}, \zeta_{2}$ and $\zeta_{3}$ above; this is done in Section 6. In the vector case, these arguments require a useful recent estimate of [Kurzke and Spirn 2009], without which we would not be able to establish the full energy-momentum tensor estimate (1-21).

1.4. Some examples. It is well known that the timelike minimal surface equation for $(1+1)$-dimensional surfaces in $\mathbb{R}^{1+N}$ is explicitly solvable for every $N \geq 2$. In particular, if $a: \mathbb{R} \rightarrow \mathbb{R}^{N}$ and $b: \mathbb{R} \rightarrow \mathbb{R}^{N}$ are smooth maps such that $\left|a^{\prime}\right|=\left|b^{\prime}\right|=1$, then the function

$$
X(s, t):=(t, x(s, t)) \quad \text { with } \quad x(s, t):=\frac{1}{2}(a(s+t)+b(s-t))
$$

parametrizes a surface that satisfies the timelike minimal surface equation wherever it is smooth. (See, for example, the exposition in [Vilenkin and Shellard 1994, Chapter 6].) From this one can deduce, in particular, that, if $g: \mathbb{R} \rightarrow \mathbb{R}^{k}$ is any smooth function (where $k=N-1$ ), then

$$
\Gamma:=\{(t, s, g(s-t)): t, s \in \mathbb{R}\}
$$

is a $(1+1)$-dimensional minimal surface in $\mathbb{R}^{1+N}$. For a timelike minimal surface $\Gamma$ of this very simple form, it turns out that there are corresponding solutions of the nonlinear wave equation (1-1) that exactly follow $\Gamma$. Indeed, if $q: \mathbb{R}^{k} \rightarrow \mathbb{R}^{k}$ is any smooth solution of

$$
-\Delta q+\left(q^{2}-1\right) q=0
$$

then, after writing $x \in \mathbb{R}^{N}=\mathbb{R}^{1+k}$ as $\left(x^{1}, x^{v}\right) \in \mathbb{R} \times \mathbb{R}^{k}$,

$$
u(t, x):=q\left(\frac{x^{\nu}-g\left(x^{1}-t\right)}{\varepsilon}\right)
$$

solves (1-1) in all of $\mathbb{R}^{1+N}$.

In particular, consider a family of surfaces $\left(\Gamma^{\varepsilon}\right)_{\varepsilon \in(0,1]}$ of the form (1-34) associated with a sequence of smooth rapidly oscillating functions $\left(g_{\varepsilon}\right)$, converging weakly in $H^{1}$ to a limiting function $g_{0}$. Although $\Gamma^{\varepsilon}$ converges in Hausdorff distance to the minimal surface $\Gamma_{0}$ associated via (1-34) with the function $g_{0}$, one can arrange the oscillation in such a way that $\mathscr{T}\left(\Gamma^{\varepsilon}\right)$ converges weakly to a limiting measure that is not equal to $\mathscr{T}\left(\Gamma_{0}\right)$. (This is a simple special case of the phenomenon known in the cosmology literature as "wiggly strings"; see again [Vilenkin and Shellard 1994, chapter 6]. Related issues are also discussed in [Neu 1990].)

\footnotetext{
${ }^{2}$ Take $a(s)=(s, 0)$ and $b(s)$ of the form $b=\left(\sigma(s), h(\sigma(s))\right.$, for $h: \mathbb{R} \rightarrow \mathbb{R}^{k-1}$ smooth, and $\sigma$ strictly increasing and adjusted so that $\left|b^{\prime}\right| \equiv 1$. Then, a change of variables shows that the surface parametrized by $x(s, t)$ can be written in the form (1-34), if $g$ is defined by requiring that $\frac{1}{2} h(\sigma(r))=g\left(\frac{1}{2}(\sigma(r)+r)\right)$ for all $r$. One can check that any smooth $g$ can be realized in this way.
} 
To illustrate this in detail, let us for simplicity assume that $k=1$ and $g_{0}=0$. One can check that, if $u_{\varepsilon}$ is the solution of the form (1-35) associated with $g_{\varepsilon}$, then (using notation defined in Section 2.3)

$$
\mathscr{T}_{\varepsilon}\left(u_{\varepsilon}\right)=\frac{1}{\varepsilon^{2}} q^{\prime 2}\left(\begin{array}{ccc}
1+g_{\varepsilon}^{\prime 2} & -g_{\varepsilon}^{\prime 2} & g_{\varepsilon}^{\prime} \\
g_{\varepsilon}^{\prime 2} & 1-g_{\varepsilon}^{\prime 2} & g_{\varepsilon}^{\prime} \\
-g_{\varepsilon}^{\prime} & g_{\varepsilon}^{\prime} & 0
\end{array}\right) \quad \text { and } \quad \mathcal{T}\left(\Gamma_{0}\right)=\left(\begin{array}{ccc}
1 & 0 & 0 \\
0 & 1 & 0 \\
0 & 0 & 0
\end{array}\right) \mathscr{H}^{1+1}\left\llcorner\Gamma_{0}\right.
$$

From these it is easy to see that, unless $g_{\varepsilon} \rightarrow g_{0}=0$ strongly in $H_{\text {loc }}^{1}(\mathbb{R}),\left(\varepsilon / \kappa_{1}\right) \mathscr{T}_{\varepsilon}\left(u_{\varepsilon}\right)$ converges to a limit that does not equal $\mathscr{T}\left(\Gamma_{0}\right)$. One can further check that this limit in general is not the energy-momentum tensor for any smooth string.

\section{Notation and assumptions}

2.1. General notation. We will write $B(\rho)$ to denote an open ball of radius $\rho$ centered at the origin.

In order to emphasize the parallels between the two cases we consider, we will use the same notation for $k=1$ and $k=2$, normally without indicating the dependence on $k$. For example, we will write

$$
\delta_{\varepsilon}:= \begin{cases}\varepsilon / \kappa_{1} & \text { when } k=1, \text { for } \kappa_{1} \text { defined in }(1-11) ; \\ (\pi|\ln \varepsilon|)^{-1} & \text { for } k=2 .\end{cases}
$$

Similarly, $\mathscr{D}$ and $\mathscr{D}_{\mathcal{V}}$ will have different meanings in the cases $k=1$ and $k=2$; see (3-1)-(3-3) and (5-1)-(5-2).

Throughout this work, we consider $(1+n)$-dimensional submanifolds in $(1+N)$-dimensional Minkowski space. We will always write $k=N-n$ for the codimension of the manifold. The same number $k$ is also the dimension of the target space for the semilinear wave equation (1-1).

A parametric $(1+n)$-dimensional submanifold $\Gamma$ of $\mathbb{R}^{1+N}$ is a submanifold described as the image of a smooth map $H: U \rightarrow \mathbb{R}^{1+N}$, where $U$ is an open subset of $\mathbb{R}^{1+n}$. We will generally assume that this map $H$ is injective. Given a map $H$ parametrizing a surface $\Gamma$, we will often define a map $\psi: U \times\left(\right.$ small ball in $\left.\mathbb{R}^{k}\right) \rightarrow \mathbb{R}^{1+N}$ that parametrizes a neighborhood of $\Gamma$ and agrees with $H$ on $U \times\{0\}$. In this situation, we will typically write points in $U \times \mathbb{R}^{k} \subset \mathbb{R}^{1+N}$ in the form

$$
y=\left(y^{\tau}, y^{\nu}\right) \quad \text { with } y^{\tau}=\left(y^{0}, \ldots, y^{n}\right) \in U \text { and } y^{\nu}=\left(y^{n+1}, \ldots, y^{N}\right) \in \mathbb{R}^{k} .
$$

The superscripts stand for "tangential" and "normal", respectively. We will also sometimes use the alternative notation

$$
y^{v}=\left(y^{v, 1}, \ldots, y^{v, k}\right)
$$

for $y^{\nu}$. We will always arrange that $y^{0}$ is a timelike coordinate, and we will often write $y^{\tau^{\prime}}=\left(y^{1}, \ldots y^{n}\right)$ and $y^{\prime}:=\left(y^{\tau \prime}, y^{\nu}\right)$, so that a "prime" denotes spatial variables only.

For notational consistency, we may sometimes write $y^{\tau}$ to denote a point $\left(y^{0}, \ldots, y^{n}\right) \in U \subset \mathbb{R}^{1+n}$ even when there are no normal $y^{v}$ variables present. We may also write, for example, $\mathbb{R}_{v}^{k}$ to denote a copy of $\mathbb{R}^{k}$ that should be thought of as being in the normal $y^{v}$ variables, and we will write $B_{v}(\rho):=$ $\left\{y^{v} \in \mathbb{R}_{v}^{k}:\left|y^{v}\right|<\rho\right\}$, where $k$ should be clear from the context. We will generally write $\nabla$ to denote the gradient in spatial directions only, and $D$ to denote the spacetime gradient, so that $D=\left(\partial_{t}, \nabla\right)$. When using the notation (2-2), we will similarly write $D=\left(D_{\tau}, \nabla_{v}\right)=\left(\partial_{y^{0}}, \nabla_{\tau}, \nabla_{v}\right)$, where for example $\nabla_{v}=\left(\partial_{y^{n+1}}, \ldots, \partial_{y^{N}}\right)$. 
We write $\eta=\left(\eta_{\alpha \beta}\right)=\left(\eta^{\alpha \beta}\right)$ to denote the diagonal matrix $\operatorname{diag}(-1,1, \ldots, 1)$.

We normally follow the convention that Latin indices $i, j, k$ run from 1 to $N$, while Greek indices $\alpha, \beta, \gamma$ run from 0 to $N$; we sum over repeated upper and lower indices. When summing implicitly over the $(t, x)$ variables, we will identify $x^{0}$ with $t$.

2.2. Assumptions and notation related to timelike minimal surfaces. A parametric submanifold is said to be timelike if $\gamma(D H):=\operatorname{det}\left(D H^{T} \eta D H\right)<0$ at every point of $U$. The Minkowski area of a timelike parametric submanifold is defined to be

$$
\mathscr{L}(H):=\int_{U} \sqrt{-\gamma}
$$

A timelike submanifold $\Gamma=\operatorname{Image}(H)$ is said to be a timelike minimal surface if $H$ is a critical point of $\mathscr{L}$. (The terminology, although standard, is misleading, as a minimal surface $\Gamma$ is in general not a minimizer or local minimizer of $\mathscr{L}$.)

Our main results all involve a timelike minimal surface $\Gamma$ that is the image of a smooth, injective map $H:(-T, T) \times \mathbb{\mathbb { T }}^{n} \rightarrow(-T, T) \times \mathbb{R}^{N}$ of the form

$$
H\left(y^{0}, \ldots, y^{n}\right)=\left(y^{0}, h\left(y^{0}, \ldots, y^{n}\right)\right) \quad \text { for some smooth } h:(-T, T) \times \mathbb{T}^{n} \rightarrow \mathbb{R}^{N},
$$

where $\mathbb{T}^{n}$ denotes the $n$-dimensional torus, thought of as the periodic unit cube (so that $\mathscr{H}^{n}\left(\mathbb{T}^{n}\right)=1$ ). We will require that our parametrization satisfies ${ }^{3}$

$$
H_{y_{0}}^{T} \eta H_{y_{i}}=h_{y_{0}} \cdot h_{y_{i}}=0 \text { for } i>0,
$$

where, here and throughout, we view $H$ and $h$ as column vectors. One can easily check that, if $\Gamma$ is a timelike parametric submanifold given as the image of a map $H$ satisfying (2-5) and (2-6), then, for any $T_{1}<T$, there exists some $\alpha>0$ such that

$$
H_{y_{0}}^{T} \eta H_{y_{0}}=-1+\left|h_{y_{0}}\right|^{2} \leq-\alpha \quad \text { and } \quad \nabla H^{T} \nabla H \geq \alpha I_{n} \quad \text { for all } y^{\tau} \in\left(-T_{1}, T_{1}\right) \times \mathbb{T}^{n} .
$$

2.3. Energy-momentum tensors. Among other results, we establish a relationship between the energymomentum tensors for a codimension- $k$ timelike Minkowski minimal surface in $\mathbb{R}^{1+N}$ and its counterpart for the semilinear wave equation (1-1) for a function $\mathbb{R}^{1+N} \rightarrow \mathbb{R}^{k}$ with $0<\varepsilon \ll 1$. We recall the definitions:

If $u$ solves (1-1), then $\mathscr{T}_{\varepsilon}(u)$ is defined to be the tensor whose components are

$$
\mathscr{T}_{\varepsilon, \beta}^{\alpha}(u):=\delta_{\beta}^{\alpha}\left(\frac{1}{2} \eta^{\gamma \delta} u_{x^{\gamma}} \cdot u_{x^{\delta}}+\frac{1}{\varepsilon^{2}} F(u)\right)-\eta^{\alpha \gamma} u_{x^{\gamma}} \cdot u_{x^{\beta}} .
$$

Here, $\left(\eta^{\alpha \beta}\right)=\operatorname{diag}(-1,1, \ldots, 1)$ as usual. (We deviate from convention in taking $\mathscr{T}_{\varepsilon}(u)$ and $\mathscr{T}(\Gamma)$ to be tensors of type $(1,1)$ rather than of type $(0,2)$; to recover the standard definition, one must lower an index.)

And, if $\Gamma$ is a timelike minimal surface, we define $\mathscr{T}(\Gamma)$ to be the tensor whose components are the

\footnotetext{
${ }^{3}$ Assumption (2-6) does not entail any loss of generality. Indeed, for $H$ of the form (2-5), we can always achieve (2-6) by replacing $h$ by a function $\tilde{h}$ of the form $\tilde{h}\left(y_{0}, \ldots, y_{n}\right)=h\left(y_{0}, \Psi\left(y_{0}, \ldots, y_{n}\right)\right)$ for a suitable $\Psi:(-T, T) \times \mathbb{T}^{n} \rightarrow(-T, T) \times \mathbb{T}^{n}$. The suitable $\Psi$ can be found by making the ansatz $\tilde{H}(y)=\left(y_{0}, \tilde{h}(y)\right)$ for $\tilde{h}$, and substituting into (2-6). This yields an ordinary differential equation for $\Psi$ that we can supplement with the initial conditions $\Psi\left(0, y^{\prime}\right)=y^{\prime}$ and then solve by appealing to standard theory.
} 
signed measures

$$
\mathscr{T}_{\beta}^{\alpha}(\Gamma)(A):=\int_{A} P_{\beta}^{\alpha}(t, x) d \lambda_{\Gamma},
$$

where $\lambda_{\Gamma}$ denotes the Minkowski area density of $\Gamma$, and where $P(t, x)=\left(P_{\beta}^{\alpha}(t, x)\right)$ is the matrix corresponding to the Minkowski orthogonal projection onto $T_{(t, x)} \Gamma$, for $\lambda_{\Gamma}$-a.e. $(t, x) \in \Gamma$. That is, if $H: U \subset \mathbb{R}^{1+n} \rightarrow \mathcal{U} \subset \mathbb{R}^{1+N}$ is a smooth injective map such that $\Gamma=H(U)$, then $\lambda_{\Gamma}$ denotes the measure on $u$ defined by

$$
\int_{\mathbb{R}^{1+N}} f(x) d \lambda_{\Gamma}:=\int_{U} f\left(H\left(y^{\tau}\right)\right) \sqrt{-\gamma\left(y^{\tau}\right)} d y^{\tau} .
$$

where as before $\gamma=\operatorname{det}\left(D H^{T} \eta D H\right)$. (It is easy to check that $\lambda_{\Gamma}$ depends only on $\Gamma$.) $P=P(t, x)$ is characterized by

$$
P_{\beta}^{\alpha} v^{\beta}=v^{\alpha} \quad \text { for } v \in T_{(t, x)} \Gamma \quad \text { and } \quad P_{\beta}^{\alpha} w^{\beta}=0 \quad \text { if } w^{T} \eta v=0 \text { for all } v \in T_{(t, x)} \Gamma .
$$

For both models, the energy-momentum tensor may be obtained by considering variations of the relevant action functional with respect to suitable one-parameter families of diffeomorphisms. We recall this in some detail for $\mathscr{T}(\Gamma)$, as we will need to refer to this later:

Lemma 3. Suppose that $H: U \subset \mathbb{R}^{1+n} \rightarrow \cup \subset \mathbb{R}^{1+N}$ is a smooth injective map whose image $\Gamma:=H(U)$ is a timelike surface. Given $\tau \in C_{c}^{\infty}\left(\mathcal{U} ; \mathbb{R}^{1+N}\right)$, define $\Phi_{\sigma}(x):=x+\sigma \tau(x)$. We have

$$
\left.\frac{d}{d \sigma} \mathscr{L}\left(\Phi_{\sigma} \circ H\right)\right|_{\sigma=0}=\int_{U_{U}} \tau_{x^{\alpha}}^{\beta}(x) P_{\beta}^{\alpha} d \lambda_{\Gamma}=\int_{U_{U}} \tau_{x^{\alpha}}^{\beta}(x) d \mathscr{T}_{\beta}^{\alpha}(\Gamma) .
$$

Note that (2-10) exactly parallels the well-known first variation formula in the Euclidean case, in which $\lambda_{\Gamma}$ is replaced by the restriction to $\Gamma$ of the Hausdorff measure of the suitable dimension, and $P_{\beta}^{\alpha}$ is replaced by the orthogonal projection with respect to the Euclidean inner product.

Exactly parallel to $(2-10), \mathscr{T}_{\varepsilon}(u)$ arises from domain variations of the action functional, say $\mathscr{A}_{\varepsilon}$, whose Euler-Lagrange equation is (1-1); see for example [Shatah and Struwe 1998] for the proof. Thus, the results (1-15) and (1-21) assert that the first variation of $\mathscr{A}_{\varepsilon}$ (with respect to domain variations) at the critical point $u$ is close (in a weak topology, and after suitable rescaling) to the first variation of $\mathscr{L}$ at the associated timelike minimal surface $\Gamma$.

We present the standard calculation that leads to (2-10), since we will need it later:

Proof of Lemma 3. We will write $H_{\sigma}:=\Phi_{\sigma} \circ H$,

$$
\begin{aligned}
\gamma_{\sigma, a b} & =H_{\sigma, y^{a}}^{T} \eta H_{\sigma, y^{b}}=H_{\sigma, y^{a}}^{\alpha} \eta_{\alpha \beta} H_{\sigma, y^{b}}^{\beta}, \\
\left(\gamma_{\sigma}^{a b}\right) & =\left(\gamma_{\sigma, a b}\right)^{-1}, \quad \text { and } \\
\gamma_{\sigma} & =\operatorname{det}\left(\gamma_{\sigma, a b}\right),
\end{aligned}
$$

where the indices $a, b$ run from 0 to $n$ and $\alpha, \beta$, as usual, run from 0 to $N$. Using the fact that

$$
\frac{d}{d \sigma} \gamma_{\sigma}=\gamma_{\sigma} \gamma_{\sigma}^{a b} \frac{d}{d \sigma} \gamma_{\sigma, a b}
$$


we find that

$$
\begin{aligned}
\left.\frac{d}{d \sigma} \mathscr{L}\left(H_{\sigma}\right)\right|_{\sigma=0}=\left.\frac{d}{d \sigma} \int_{U} \sqrt{-\gamma_{\sigma}}\right|_{\sigma=0} & =\int_{U}\left(\tau^{\beta} \circ H\right)_{y^{a}} \eta_{\beta \delta} H_{y^{b}}^{\delta} \gamma^{a b} \sqrt{-\gamma} d y^{\tau} \\
& =\int_{U}\left(\tau_{x^{\alpha}}^{\beta} \circ H\right) H_{y^{a}}^{\alpha} \eta_{\beta \delta} H_{y^{b}}^{\delta} \gamma^{a b} \sqrt{-\gamma} d y^{\tau} \\
& =\int_{U} \tau_{x^{\alpha}}^{\beta}(t, x) P_{\beta}^{\alpha}(t, x) d \lambda_{\Gamma}
\end{aligned}
$$

where

$$
P_{\beta}^{\alpha}\left(H\left(y^{\tau}\right)\right):=H_{y^{a}}^{\alpha}\left(y^{\tau}\right) \gamma^{a b}\left(y^{\tau}\right) H_{y^{b}}^{\delta}\left(y^{\tau}\right) \eta_{\delta \beta} .
$$

Note that $P_{\beta}^{\alpha}$ is defined for $\lambda_{\Gamma}$-a.e. $(t, x)$, so the above integral makes sense. In order to complete the proof, we must check that $P_{\beta}^{\alpha}(t, x)$ is the orthogonal projection onto $T_{(t, x)} \Gamma$. To see this, first note that, at any $y^{\tau} \in \mathbb{R}^{1+n}$,

$$
\left(P H_{y^{c}}\right)^{\alpha}=P_{\beta}^{\alpha} H_{y^{c}}^{\beta}=H_{y^{a}}^{\alpha} \gamma^{a b} H_{y^{b}}^{\delta} \eta_{\delta \beta} H_{y^{c}}^{\beta}=H_{y^{a}}^{\alpha} \gamma^{a b} \gamma_{b c}=H_{y^{a}}^{\alpha} \delta_{c}^{a}=H_{y^{c}}^{\alpha} .
$$

Thus, $P H_{y^{c}}=H_{y^{c}}$. And, if $v$ is orthogonal to $H_{y^{b}}$ for all $b$, then

$$
(P v)^{\alpha}=P_{\beta}^{\alpha} v^{\beta}=H_{y^{a}}^{\alpha} \gamma^{a b} H_{y^{b}}^{\delta} \eta_{\delta \beta} v^{\beta}=0,
$$

since the orthogonality of $v$ means exactly that $H_{y^{b}}^{\delta} \eta_{\delta \beta} v^{\beta}=0$ for every $b$. Since $T_{(t, x)} \Gamma$ at $(t, x)=H\left(y^{\tau}\right)$ is spanned by $\left\{H_{y^{b}}\left(y^{\tau}\right)\right\}_{b=0}^{n}$, the above calculations state exactly that $P(t, x)$ is the matrix corresponding to orthogonal projection onto $T_{(t, x)} \Gamma$.

2.4. Change of variables. We next define the change of variables that, as mentioned earlier, is the starting point of our argument. We will use the notation (2-2).

We assume, as always, that $\Gamma$ is a smooth timelike minimal surface, given as the image ${ }^{4}$ of a smooth injective map $H:(-T, T) \times \mathbb{T}^{n} \rightarrow \mathbb{R}^{1+N}$ satisfying (2-5) and (2-7). For this section, we allow $k=N-n$ to be an arbitrary positive integer, since all the proofs for $k=2$ apply without change to $k \geq 3$. (The case $k=1$ is simpler.) Although we do not use them in this paper, the results for $k \geq 3$ may be useful for problems such as the dynamics of defects in certain nonabelian gauge theories.

First, we fix smooth maps $\bar{v}_{i}:(-T, T) \times \mathbb{T}^{n} \rightarrow \mathbb{R}^{1+N}$ for $i=1, \ldots, k$, such that

$$
\bar{v}_{i}^{T} \eta \bar{v}_{j}=\delta_{i j} \quad \text { and } \quad H_{y^{\alpha}}^{T} \eta \bar{v}_{i}=0 \quad \text { in }(-T, T) \times \mathbb{T}^{n} \rightarrow \mathbb{R}^{1+N},
$$

for all $i, j \in\{1, \ldots, k\}$ and $\alpha \in\{0, \ldots, n\}$. (Here and throughout the paper, we are thinking of $\bar{v}_{i}$ as a column vector.) This states that $\left\{\bar{v}_{1}\left(y^{\tau}\right), \ldots, \bar{v}_{k}\left(y^{\tau}\right)\right\}$ form an orthonormal basis for the normal space to $\Gamma$ at $H\left(y^{\tau}\right)$, where words like "normal" and "orthonormal" are understood with respect to the Minkowski inner product, and $y^{\tau}$ denotes a generic point in $(-T, T) \times \mathbb{T}^{n}$. Note that, when $k=1$, (2-11) determines $\bar{v}_{1}$ up to a sign, whereas for $k \geq 2$ there are rotational degrees of freedom that we have not specified (and will not specify).

\footnotetext{
${ }^{4}$ All the results of this section are local, so the topology of $\Gamma$ (that is, the fact that $H$ is defined on $\left.(-T, T) \times \mathbb{T}^{n}\right)$ is irrelevant here. However, it is convenient to keep the same set-up as in the rest of the paper.
} 
Next, we define, using the notation (2-2),

$$
\psi(y):=H\left(y^{\tau}\right)+\sum_{i=1}^{k} \bar{v}_{i}\left(y^{\tau}\right) y^{n+i} .
$$

It is clear that $\psi\left(y^{\tau}, 0\right)=H\left(y^{\tau}\right)$ for all $y^{\tau} \in(-T, T) \times \mathbb{T}^{n}$.

Recall that the statement of Theorems 1 and 2 involve a number $T_{0}<T$. We henceforth fix $T_{1} \in\left(T_{0}, T\right)$, and we let $\rho_{0}>0$ be so small that

$$
\psi\left(\left\{-T_{1}\right\} \times \mathbb{T}^{n} \times B_{v}\left(\rho_{0}\right)\right) \Subset\left(-T,-T_{0}\right) \times \mathbb{R}^{N}, \quad \psi\left(\left\{T_{1}\right\} \times \mathbb{T}^{n} \times B_{v}\left(\rho_{0}\right)\right) \subset\left(T_{0}, T\right) \times \mathbb{R}^{N},
$$

and

$$
\psi \text { is injective, with smooth inverse } \varphi \text {, on }\left(-T_{1}, T_{1}\right) \times \mathbb{T}^{n} \times B_{v}\left(\rho_{0}\right) .
$$

The latter condition can be satisfied due to the inverse function theorem; indeed, we will check below that $D \psi\left(y^{\tau}, 0\right)$ is invertible for $y^{\tau} \in\left(-T_{1}, T_{1}\right) \times \mathbb{T}^{n}$. We next define

$$
\left(g_{\alpha \beta}\right)_{\alpha, \beta=0}^{N}=G:=D \psi^{T} \eta D \psi,
$$

so that $G$ represents the Minkowski metric in the $y$ coordinates. We further define $g:=\operatorname{det} G$ and $\left(g^{\alpha \beta}\right)_{\alpha, \beta=0}^{N}:=G^{-1}$, and we finally define $\left(a^{\alpha \beta}\right)_{\alpha, \beta=0}^{N}$ by

$$
a^{i j}=g^{i j} \text { if } i, j \geq 1, \quad a^{00}=-g^{00}, \quad \text { and } \quad a^{i 0}=a^{0 j}=0 \text { for } i, j=1, \ldots, N .
$$

When we write (1-1) in terms of the $y$ coordinates as in (1-22), $\left(g^{\alpha \beta}\right)$ and $g$ appear in the coefficients and $\left(a^{\alpha \beta}\right)$ appears in a natural associated energy density $e_{\varepsilon}(v)=e_{\varepsilon}(v ; G)$, defined in (1-28). We summarize the properties of $g$ and $\left(g^{\alpha \beta}\right)$ that we will use:

Proposition 4. Let $\psi, g,\left(g^{\alpha \beta}\right)$ be the functions on $\left(-T_{1}, T_{1}\right) \times \mathbb{T}^{n} \times B_{v}\left(\rho_{0}\right)$ defined above. After taking $\rho_{0}$ smaller if necessary, there exist positive constants $c \leq C$ such that

$$
\begin{aligned}
& \left\|g^{\alpha \beta}\right\|_{W^{1, \infty}} \leq C, \quad g_{y^{0}}^{\alpha \beta} \xi_{\alpha} \xi_{\beta} \leq C\left(\left|\xi_{\tau}\right|^{2}+\left|y^{\nu}\right|^{2}\left|\xi_{\nu}\right|^{2}\right), \\
& \frac{\partial_{y^{\alpha}} \sqrt{-g}}{\sqrt{-g}} g^{\alpha \beta} \xi_{\beta} \xi_{0} \leq C\left(\left|\xi_{\tau}\right|^{2}+\left|y^{\nu}\right|^{2}\left|\xi_{\nu}\right|^{2}\right), \\
& \left|g^{\alpha \beta} \xi_{\beta}\right| \leq C\left(\left|\xi_{\tau}\right|+\left|y^{\nu}\right|\left|\xi_{\nu}\right|\right) \quad \text { if } \alpha \leq n, \quad \text { and } \\
& c\left|\xi_{\tau}\right|^{2}+\left(1-C\left|y^{\nu}\right|^{2}\right)\left|\xi_{\nu}\right|^{2} \leq a^{\alpha \beta}(y) \xi_{\alpha} \xi_{\beta} \leq C\left|\xi_{\tau}\right|^{2}+\left(1+C\left|y^{\nu}\right|^{2}\right)\left|\xi_{\nu}\right|^{2}
\end{aligned}
$$

for all $y=\left(y^{\tau}, y^{\nu}\right) \in\left(-T_{1}, T_{1}\right) \times \mathbb{T}^{n} \times B_{v}\left(\rho_{0}\right)$ and $\xi=\left(\xi_{\tau}, \xi_{v}\right) \in \mathbb{R}^{1+N} \cong \mathbb{R}^{1+n} \times \mathbb{R}^{k}$. In addition,

$$
\psi_{y^{0}}^{0} \geq c \quad \text { in }\left(-T_{1}, T_{1}\right) \times \mathbb{T}^{n} \times B_{v}\left(\rho_{0}\right) .
$$

We emphasize that the main point in the proof of (2-18) is that $\nabla_{v} \sqrt{-g}=0$ when $y^{v}=0$. This is equivalent to $\Gamma$ having zero mean curvature.

We will use the notation

$$
\mathcal{N}:=\psi\left(\left(-T_{1}, T_{1}\right) \times \mathbb{T}^{n} \times B_{v}\left(\rho_{0}\right)\right) \cap\left[\left(-T_{0}, T_{0}\right) \times \mathbb{R}^{N}\right]
$$


For future use, it is convenient to fix a constant $\kappa_{2} \geq 1$ such that

$$
\left(1+\kappa_{2}\left|y^{v}\right|^{2}\right) e_{\varepsilon}(v) \geq \frac{\lambda}{2}\left|D_{\tau} v\right|^{2}+\left(1+\left|y^{v}\right|^{2}\right) e_{\varepsilon, v}(v)
$$

everywhere in $\left(-T_{1}, T_{1}\right) \times \mathbb{T}^{n} \times B_{v}\left(\rho_{0}\right)$ and for all $v \in H^{1}$, where $\varepsilon_{\varepsilon, v}$ was defined in (1-24). This is possible due to (2-20).

When $\Gamma$ is a hypersurface, we have a slightly better behavior:

Proposition 5. Suppose that $k=1$, and let $\psi, g$, and $\left(g^{\alpha \beta}\right)$ be as defined above. After taking $\rho_{0}$ smaller if necessary,

$$
g^{\alpha N}=g^{N \alpha}= \begin{cases}1 & \text { if } \alpha=N, \\ 0 & \text { otherwise }\end{cases}
$$

and, in addition, there exist positive constants $\lambda<\Lambda$ such that

$$
\lambda\left|\xi_{\tau}\right|^{2}+\left|\xi_{v}\right|^{2} \leq a^{\alpha \beta}(y) \xi_{\alpha} \xi_{\beta} \leq \Lambda\left|\xi_{\tau}\right|^{2}+\left|\xi_{v}\right|^{2}
$$

everywhere in $\left(-T_{1}, T_{1}\right) \times \mathbb{T}^{n} \times B_{v}\left(\rho_{0}\right)$.

Conclusion (2-25) is not essential, but will allow us to simplify our notation, for example by taking $\kappa_{2}=1$ in (2-23) and everywhere else that this constant occurs (for $k=1$ ).

We defer the proofs of Propositions 4 and 5 to an Appendix.

For a solution $u: \mathbb{R}^{1+N} \rightarrow \mathbb{R}^{k}$ of (1-1), we will define $v:\left(-T_{1}, T_{1}\right) \times \mathbb{T}^{n} \times B_{v}\left(\rho_{0}\right) \rightarrow \mathbb{R}^{k}$ by $v=u \circ \psi$. Then $v$ satisfies

on its domain. Here,

$$
\square_{G} v+\frac{1}{\varepsilon^{2}} f(v)=0
$$

$$
\square_{G} v=-\frac{1}{\sqrt{-g}} \partial_{y^{\alpha}}\left(\sqrt{-g} g^{\alpha \beta} \partial_{y^{\beta}} v\right) .
$$

As noted earlier, we find it convenient to write (2-26) in the form (1-22). We now derive a key differential inequality for the energy density $e_{\varepsilon}(v)$ from (1-28):

Lemma 6. If $v:\left(-T_{1}, T_{1}\right) \times \mathbb{T}^{n} \times B_{v}\left(\rho_{0}\right) \rightarrow \mathbb{R}^{k}$ is a smooth solution of (2-26), with coefficients satisfying (2-17), then

$$
\begin{aligned}
& \frac{\partial}{\partial y^{0}} e_{\varepsilon}(v) \leq C\left(\left|D_{\tau} v\right|^{2}+\left|y^{v}\right|^{2}\left|\nabla_{\nu} v\right|^{2}\right)+\nabla \cdot \varphi, \text { with } \\
& \varphi:=\left(\varphi^{1}, \ldots, \varphi^{N}\right), \quad \varphi^{i}:=g^{i \alpha} v_{y^{\alpha}} \cdot v_{y^{0}} .
\end{aligned}
$$

Proof. Multiply (1-22) by $v_{y^{0}}$, and rewrite to find that

$$
-\partial_{y^{\alpha}}\left(g^{\alpha \beta} v_{y^{\beta}} \cdot v_{y^{0}}\right)+g^{\alpha \beta} v_{y^{\beta}} \cdot v_{y^{0} y^{\alpha}}+\frac{1}{\varepsilon^{2}} F(v)_{y^{0}}=-(b \cdot D v) \cdot v_{y^{0}} .
$$

We rewrite $g^{\alpha \beta} v_{y^{\beta}} \cdot v_{y^{0} y^{\alpha}}$ as

$$
\frac{1}{2} \partial_{y^{0}}\left(g^{\alpha \beta} v_{y^{\beta}} \cdot v_{y^{\alpha}}\right)-\frac{1}{2} g_{y^{0}}^{\alpha \beta} v_{y^{\beta}} \cdot v_{y^{\alpha}} .
$$

Gathering all the terms of the form $\partial_{y^{0}}(\cdots)$ on the left-hand side, we find that

$$
\partial_{y^{0}}\left(-g^{0 \beta} v_{y^{\beta}} \cdot v_{y^{0}}+\frac{1}{2} g^{\alpha \beta} v_{y^{\alpha}} \cdot v_{y^{\beta}}+\frac{1}{\varepsilon^{2}} F(v)\right)=\partial_{y^{i}}\left(g^{i \beta} v_{y^{\beta}} \cdot v_{y^{0}}\right)-(b \cdot D v) v_{y^{0}}+\frac{1}{2} g_{y^{0}}^{\alpha \beta} v_{y^{\beta}} \cdot v_{y^{\alpha}} .
$$


The definition (2-16) of $a^{\alpha \beta}$ implies that the left-hand side is just $\partial_{y^{0}} e_{\varepsilon}(v)$. To complete the proof, we use (2-17) and (2-18) to check that the non-divergence terms on the left-hand side are bounded by $C\left(\left|D_{\tau} v\right|^{2}+\left(y^{v}\right)^{2}\left|\nabla_{v} v\right|^{2}\right)$.

As an easy consequence of Proposition 5, we obtain a quite explicit description of the signed Minkowski distance function, defined by the eikonal equation (1-12) in the case $k=1$.

Corollary 7. If $k=1, \psi$ is defined as above, and $\varphi=\left(\varphi^{0}, \ldots, \varphi^{N}\right)$ denotes the inverse of $\psi$, then $\varphi^{N}$ solves the eikonal equation (1-12) on Image $(\psi)$.

In particular, Corollary 7 shows that it makes sense to speak of the signed-distance function in the set $\mathcal{N}$ defined in (2-22).

Proof. Fix a point in the image of $\psi$, say, $(t, x)=\psi(y)$. Then, since $\eta=\eta^{-1}$,

$$
\left(g^{\alpha \beta}\right)(y)=\left[D \psi^{T}(y) \eta D \psi(y)\right]^{-1}=(D \psi)^{-1}(y) \eta(D \psi)^{-T}(y)=D \varphi(t, x) \eta D \varphi^{T}(t, x) .
$$

Thus, according to (2-24),

$$
1=g^{N N}(y)=-\left(\varphi_{t}^{N}\right)^{2}+\left|\nabla \varphi^{N}\right|^{2},
$$

so that (1-12) holds. And, it is clear that $\varphi^{N}(t, x)=0$ for $(t, x) \in \Gamma$.

In fact the curves $s \mapsto H\left(y^{\tau}\right)+s v\left(y^{\tau}\right)=\psi\left(y^{\tau}, s\right)$ are exactly the characteristic curves for the eikonal equation (1-12).

The eikonal equation (1-12) determines the distance function $d$ only up to a sign; we will always choose to identify $d$ with $\varphi^{N}$ (so that our choice of a sign is ultimately determined by our choice of the sign for the unit normal $v$.) Then, it follows that

$$
d(\psi(y))=y^{N} \quad \text { for } y \in\left(-T_{1}, T_{1}\right) \times \mathbb{T}^{n} \times B_{v}\left(\rho_{0}\right),
$$

2.5. Initial data. In this section, we describe our general assumptions on the initial data.

We will eventually combine estimates for $v=u \circ \psi$ on $\left(-T_{1}, T_{1}\right) \times \mathbb{T}^{n} \times B_{v}\left(\rho_{0}\right)$ (which we use to control the behavior of $u$ near $\Gamma$ ) with standard energy estimates for (1-1) away from $\Gamma$. We start by making a number of smallness assumptions, in all of which a parameter $\zeta_{0}$ appears. We will prove below that one can find data for which $\zeta_{0} \approx \varepsilon^{2}$ when $k=1$, and $\zeta_{0} \approx|\ln \varepsilon|^{-1}$ when $k=2$. Although we omit the proof, it is in fact true that one cannot find data satisfying our assumptions with $\zeta_{0} \ll \varepsilon^{2}$ (for $k=1$ ) or $\zeta_{0} \ll|\ln \varepsilon|^{-1}$. We, therefore, will assume that

$$
\zeta_{0} \geq \varepsilon^{2} \quad \text { if } k=1, \quad \zeta_{0} \geq|\ln \varepsilon|^{-1} \quad \text { if } k=2 .
$$

This is convenient, as it will enable us to absorb small error terms into expressions of the form $C \zeta_{0}$.

Our first assumption is that the energy is small away from $\Gamma_{0}$ :

$$
\left.\delta_{\varepsilon} \int_{\left\{x \in \mathbb{R}^{N}:(0, x) \notin \operatorname{image}(\psi)\right\}} e_{\varepsilon}(u) d x\right|_{t=0} \leq \zeta_{0}
$$

where $e_{\varepsilon}(u)=e_{\varepsilon}(u ; \eta)$ is defined in (1-10), and $\delta_{\varepsilon}=\delta_{\varepsilon}(k)$ is defined in (2-1).

Near $\Gamma_{0}$ it is convenient to state our assumptions in terms of $v=u \circ \psi$. Note that the initial data for $u$ at $t=0$ corresponds to data for $v$ on a hypersurface that does not, in general, have the form $\left\{y_{0}=\right.$ constant $\}$. This hypersurface is described next: 
Lemma 8. There exists a Lipschitz function $b: \mathbb{T}^{n} \times B_{v}\left(\rho_{0}\right) \rightarrow \mathbb{R}$ such that, for arbitrary $y=\left(y_{0}, y^{\prime}\right)$ in $\left(-T_{1}, T_{1}\right) \times \mathbb{T}^{n} \times B_{v}\left(\rho_{0}\right)$,

$$
\psi\left(y_{0}, y^{\prime}\right) \in\{0\} \times \mathbb{R}^{N} \quad \text { if and only if } \quad y_{0}=b\left(y^{\prime}\right) .
$$

Moreover, $\|\nabla b\|_{\infty} \leq C$.

Proof. Fix $y^{\prime} \in \mathbb{T}^{n} \times B_{v}\left(\rho_{0}\right)$ and, for $s \in\left(-T_{1}, T_{1}\right)$, let $y(s):=\left(s, y^{\prime}\right)$ and let $X(s)=\psi(y(s)) \in \mathbb{R}^{1+N}$. To prove that $\psi^{-1}\left(\{0\} \times \mathbb{R}^{N}\right)$ is the graph of a function, we need to show that $y(s)$ intersects $\psi^{-1}\left(\{0\} \times \mathbb{R}^{N}\right)$ exactly once or, equivalently, that $X(s)$ intersects $\{0\} \times \mathbb{R}^{N}$ for exactly one value of $s$. To prove this, note that the definition of $G$ and (2-7) imply that, after taking $\rho_{0}$ smaller if necessary,

$$
X^{\prime}(s)^{T} \eta X^{\prime}(s)=y^{\prime}(s)^{T} G(y(s)) y^{\prime}(s)=g_{00}(y(s))<0
$$

for every $s$. Thus, $s \mapsto X(s)=\left(X^{0}(s), X^{\prime}(s)\right)$ is a timelike curve, from which the claim is obvious. It follows that there exists a function $b$ satisfying (2-32). Then, by differentiating the identity $\psi^{0}\left(b\left(y^{\prime}\right), y^{\prime}\right)=0$, we find that $\psi_{y^{0}}^{0}\left(b\left(y^{\prime}\right), y^{\prime}\right) \nabla b\left(y^{\prime}\right)+\nabla \psi^{0}\left(b\left(y^{\prime}\right), y^{\prime}\right)=0$. We know from (2-21) that $\psi_{y^{0}}^{0}$ is bounded away from 0 , and this, together with the smoothness of $\psi^{0}$, implies that $\|\nabla b\|_{\infty} \leq C$.

Using the lemma, we define

$$
v_{0}\left(y^{\prime}\right):=v\left(b\left(y^{\prime}\right), y^{\prime}\right) \text { for } y^{\prime} \in \mathbb{T}^{n} \times B_{v}\left(\rho_{0}\right) .
$$

Our next assumptions specify that the energy near $\Gamma_{0}$ is small, in the frame that moves with $\Gamma$ :

$$
\begin{aligned}
& \delta_{\varepsilon} \int_{\mathbb{T}^{n} \times B_{v}\left(\rho_{0}\right)}\left(1+\kappa_{2}\left|y^{v}\right|^{2}\right) e_{\varepsilon}\left(v_{0} ; G\right) d y^{\prime}-1 \leq \zeta_{0}, \\
& \delta_{\varepsilon} \int_{\mathbb{T}^{n} \times B_{v}\left(\rho_{0}\right)}\left(\left|v_{y^{0}}\right|^{2}+\left|v_{y^{0}}\right|\left|\nabla_{v} v_{0}\right|\right)\left(b\left(y^{\prime}\right), y^{\prime}\right) d y^{\prime} \leq \zeta_{0} .
\end{aligned}
$$

Finally, using notation discussed in the Introduction and defined in (3-1) for $k=1$, and in (5-1) and (5-3) for $k=2$, we require that

$$
\mathscr{D}\left(v_{0} ; \rho_{0}\right) \leq \zeta_{0}
$$

This specifies that the initial profile possesses a defect — that is, an interface or vortex - near $\Gamma_{0}$.

Note that conditions (2-31) and (2-34)-(2-36) are always satisfied if we define $\zeta_{0}$ to be the maximum of the left-hand sides of these inequalities. The smallest possible values of $\zeta_{0}$ depend on $k$ and, as mentioned earlier, account for the fact that our conclusions for $k=1$ are stronger than for $k=2$.

Lemma 9. In the scalar case $(k=1)$, there exist initial data $\left.\left(u, u_{t}\right)\right|_{t=0} \in \dot{H}^{1} \times L^{2}\left(\mathbb{R}^{N}\right)$ for (1-1), satisfying conditions (2-31)-(2-36) with $\zeta_{0}=C \varepsilon^{2}$, and such that

$$
\int_{\mathcal{N}_{0}}\left(u(0, x)-q\left(\frac{d(0, x)}{\varepsilon}\right)\right)^{2} \leq C \varepsilon, \quad \text { where } \mathcal{N}_{0}=\left\{x \in \mathbb{R}^{N}:(0, x) \in \mathcal{N}\right\} .
$$

In the vector $(k=2)$ case, there exist initial data $\left.\left(u, u_{t}\right)\right|_{t=0} \in \dot{H}^{1} \times L^{2}\left(\mathbb{R}^{N} ; \mathbb{R}^{2}\right)$ for $(1-1)$, satisfying conditions (2-31)-(2-36) with $\zeta_{0}=C|\ln \varepsilon|^{-1}$.

Although we do not prove it, these scalings for $\zeta_{0}$ are, in fact, optimal. 
Proof. In both cases, $k=1$ and $k=2$, we define a function $U$ in Image $(\psi)$ such that

$$
U \circ \psi=\tilde{q}\left(\frac{y^{v}}{\varepsilon}\right),
$$

where $\tilde{q}: \mathbb{R}^{k} \rightarrow \mathbb{R}^{k}$ is a nearly optimal profile. We then require that

$$
u(0, x)=U(0, x) \quad \text { and } \quad u_{t}(0, x)=U_{t}(0, x) \quad \text { in } \mathcal{N}_{0},
$$

and we verify (2-34)-(2-36). (Note that (2-29) then implies that $u(x, 0)=\tilde{q}(d / \varepsilon)$ when $k=1$, which will make (2-37) obvious.) Finally, we argue that $u(0, \cdot)$ can be extended to $\mathbb{R}^{N} \backslash \mathcal{N}_{0}$ such that (2-31) holds.

Case $k=1$ : By integrating the equation (1-5) solved by $q$, and using the boundary conditions at $\pm \infty$, one finds that $q^{\prime}=\sqrt{2 F(q)}$ and, hence, that

$$
\int_{\mathbb{R}} \frac{1}{2} q^{\prime 2}+F(q) d x=\int_{\mathbb{R}} \sqrt{2 F(q)} q^{\prime}(s) d s=\int_{-1}^{1} \sqrt{2 F(s)} d s=\kappa_{1} .
$$

Using (1-5) and (1-9), standard ODE arguments show that, for suitable constants,

$$
\left|q^{\prime}(s)\right|+|q(s)-\operatorname{sign}(s)| \leq C e^{-c|s|} \quad \text { for all } s .
$$

It follows that, given $\varepsilon>0$, we can find a function $\tilde{q}$ such that $\tilde{q}(s / \varepsilon)=q(s / \varepsilon)$ if $|s|<\frac{1}{2} \rho_{0}$, and

$$
\tilde{q}\left(\frac{s}{\varepsilon}\right)=q\left(\frac{s}{\varepsilon}\right) \quad \text { if }|s|<\frac{1}{3} \rho_{0}, \quad \tilde{q}\left(\frac{s}{\varepsilon}\right)=\operatorname{sign}(s) \quad \text { if }|s|>\frac{2}{3} \rho_{0}, \quad\|\tilde{q}-q\|_{W^{1, \infty}} \leq C e^{-c / \varepsilon},
$$

and

$$
\kappa_{1}<\int_{-\rho_{0} / \varepsilon}^{\rho_{0} / \varepsilon} t \frac{1}{2} \tilde{q}^{\prime 2}+F(\tilde{q}) d x \leq \kappa_{1}+C e^{-c / \varepsilon} .
$$

Now, define $U$ as in (2-38) and define $\left.u\right|_{t=0}$ near $\Gamma_{0}$ by (2-39). Then, by construction, $v_{0}$ as defined in (2-33) is given by $v_{0}(y)=\tilde{q}\left(y^{N} / \varepsilon\right)$, and $v_{y_{0}}=0$. The latter fact immediately implies that (2-35) holds, and (2-31) and (2-35) are easily verified. For example, the explicit form of $v_{0}$ and (2-25) imply that $e_{\varepsilon}\left(v_{0} ; G\right)=\frac{1}{2} \tilde{q}^{\prime 2}\left(y^{\nu} / \varepsilon\right)+\varepsilon^{-2} F\left(\tilde{q}\left(y^{v} / \varepsilon\right)\right)$. Then, recalling that $\delta_{\varepsilon}=\varepsilon / \kappa_{1}$, we infer from (2-41) and the change of variables $y^{N} / \varepsilon \mapsto y^{N}$ that

$$
\delta_{\varepsilon} \int_{\mathbb{T}^{n} \times B_{v}\left(\rho_{0}\right)}\left(1+\kappa_{2}\left|y^{\nu}\right|^{2}\right) e_{\varepsilon}\left(v_{0} ; G\right) d y^{\prime}-1 \leq C \varepsilon^{2} \int_{-\rho_{0} / \varepsilon}^{\rho_{0} / \varepsilon}\left(\frac{\tilde{q}^{\prime 2}}{2}+F(\tilde{q})\right)\left(y^{N}\right)^{2} d y^{N}+C e^{-c / \varepsilon} .
$$

The exponential decay of $q$ implies that $\int_{R}\left(\frac{1}{2} \tilde{q}^{\prime 2}+F(\tilde{q})\right)\left(y^{N}\right)^{2} d y^{N} \leq C$ independently of $\varepsilon$, and (2-34) follows, with $\zeta_{0}=C \varepsilon^{2}$. The verifications of (2-35) and (2-36) are similar and a little easier.

Finally, on $\mathbb{R}^{N} \backslash \mathcal{N}_{0}$, we set $u_{t}(0, \cdot) \equiv 0$, and we require that $u(0, \cdot)= \pm 1$ and that $u$ be continuous (hence, smooth) across $\partial \mathcal{N}_{0}$. This can be done, since $\mathbb{R}^{N} \backslash \Gamma_{0}$ consists of two components, one of which meets $\mathcal{N}_{0}$ where $d=\rho_{0}$ (and, hence, $u=1$ ), and the other where $d=-\rho_{0}$. (Here, we have used the fact that $\rho_{0}$ is sufficiently small; see (2-13).) 
Case $k=2$ : In this case, we may define $\tilde{q}(s)=s \min \{1,1 /|s|\}$ for $s \in \mathbb{R}^{2}$, and go on to make the definitions (2-38) and (2-39) as above, so that $v_{0}(y)=\tilde{q}\left(y^{v} / \varepsilon\right)$. Then, an easy calculation shows that

$$
\frac{1}{\pi|\ln \varepsilon|} \int_{B_{v}\left(\rho_{0} / \varepsilon\right)} \frac{1}{2}|\nabla \tilde{q}|^{2}+F(\tilde{q}) d s \leq 1+C|\ln \varepsilon|^{-1} .
$$

This plays a role analogous to (2-41) above, and allows us to verify along the previous lines (but using (2-20) in place of (2-25)) that (2-34) holds with $\zeta_{0}=C|\ln \varepsilon|^{-1}$. As before, (2-35) follows from the fact that $v_{y^{0}}\left(b\left(y^{\prime}\right), y^{\prime}\right)=0$ in $\mathbb{T}^{n} \times B_{v}\left(\rho_{0}\right)$. One can check (2-36) directly from the definitions (see Section 5), noting that

$$
J_{\nu} v_{0}\left(y^{\tau}, y^{\nu}\right)= \begin{cases}\varepsilon^{-2} & \text { if }\left|y^{\nu}\right|<\varepsilon \\ 0 & \text { if }\left|y^{\nu}\right|>\varepsilon\end{cases}
$$

It remains to show that $u_{0}=U(0, \cdot)$, as defined in $\mathcal{N}_{0}$ by (2-39), can be extended to a function in $H^{1}\left(\mathbb{R}^{N}\right)$ satisfying (2-31). It is clear that we can extend $u_{0}$ by a finite-energy map in a neighborhood $\mathscr{V}$ of $\mathcal{N}_{0}$. Next, we point out that, since $\Gamma_{0}$ is a smooth, compact, oriented codimension- 2 submanifold without boundary of $\mathbb{R}^{N}$, results in [Alberti et al. 2003] imply that we may find a function $w \in H_{\text {loc }}^{1}\left(\mathbb{R}^{N} \backslash \Gamma ; \mathbb{C}\right)$ with $\int_{\mathbb{R}^{N} \backslash \mathcal{N}_{0}}|\nabla w|^{2}<\infty$, such that $|w|=1$ a.e. and, in addition, such that $J w=J\left(u_{0} /\left|u_{0}\right|\right)$ in $\Gamma_{0}$, where $J(\cdots)$ denotes the distributional Jacobian of $(\cdots)$. This implies that there exists a real-valued function $\theta \in H_{\text {loc }}^{1}\left(\mathscr{V} \backslash \Gamma_{0} ; \mathbb{R}\right)$ such that $u_{0}=\left|u_{0}\right| w e^{i \theta}$ in $\mathscr{V}$. Thus, we define $u(0, \cdot)$ globally in $\mathbb{R}^{N}$ by setting

$$
u(0, \cdot)= \begin{cases}\left|u_{0}\right| w e^{i \chi \theta} & \text { in } \mathscr{V}, \\ w & \text { in } \mathbb{R}^{N} \backslash \mathscr{V},\end{cases}
$$

where $\chi \in C_{c}^{\infty}(\mathscr{V})$ and $\chi \equiv 1$ in $\mathcal{N}_{0}$; we may set $u_{t}(0, x)=0$ outside of $\mathcal{N}_{0}$.

\section{Basic energy estimates, $k=1$}

The main result of this section - Proposition 10 below - contains the simplest case of our main estimate.

In this section and the next, we restrict our attention to the case $k=1$, so that ${ }^{5} N=n+1, y^{v}=y^{N} \in \mathbb{R}$, and $\nabla_{v}=\partial_{N}$. Thus, in this section, $B_{v}(\rho)$ denotes the interval $(-\rho, \rho)$ along the $y^{N}$ axis. We also follow other conventions for $k=1$, so that, for example, $\delta_{\varepsilon}=\varepsilon / \kappa_{1}$; see (2-1).

Throughout this section, we let $\psi$ denote the change of variables from Section 2.4 , in the case $k=1$. We also use the notation $g, g_{\alpha \beta}, g^{\alpha \beta}$, etc. from the previous section.

In the Introduction we discussed a "defect confinement" functional $\mathscr{D}$. In the case $k=1$, we define it to be

$$
\mathscr{D}(v ; \rho):=\int_{\mathbb{T}^{n} \times B_{v}(\rho)}\left|y^{v}\right|\left|v-\operatorname{sign}\left(y^{v}\right)\right|^{2} d y^{\prime}
$$

for $v: \mathbb{T}^{n} \times B_{v}(\rho) \rightarrow \mathbb{R}$. We will also write

$$
\mathscr{D}(v ; \rho)=\int_{\mathbb{T}^{n}} \mathscr{D}_{v}\left(v\left(y^{\tau \prime}\right) ; \rho\right) d y^{\tau \prime} .
$$

\footnotetext{
${ }^{5}$ Although here there is not much point in writing $y^{v}$ and $\nabla_{\nu}$ instead of $y^{N}$ and $\partial_{N}$, this notation will prove useful when we consider the vector case, and we use it here to emphasize the parallels.
} 
where $v\left(y^{\tau^{\prime}}\right)\left(y^{v}\right)=v\left(y^{\tau^{\prime}}, y^{v}\right)$, and

$$
\mathscr{D}_{\nu}(w ; \rho):=\int_{B_{v}(\rho)}\left|y^{v}\right|\left|w-\operatorname{sign}\left(y^{v}\right)\right|^{2} d y^{v} \quad \text { for } w: B_{v}(\rho) \rightarrow \mathbb{R} .
$$

Let $c_{*}$ be a constant such that

$$
\left|g^{N \alpha}(y) \xi_{\alpha} \xi_{0}\right|=\left|a^{N \alpha}(y) \xi_{\alpha} \xi_{0}\right| \leq \frac{1}{2} c_{*} a^{\alpha \beta} \xi_{\alpha} \xi_{\beta}
$$

for all $\xi \in \mathbb{R}^{1+N}$ and $y \in\left(-T_{1}, T_{1}\right) \times \mathbb{T}^{n} \times B_{v}\left(\rho_{0}\right)$.

Proposition 10. Let $v:\left(-T_{1}, T_{1}\right) \times \mathbb{T}^{n} \times B_{v}\left(\rho_{0}\right) \rightarrow \mathbb{R}$ satisfy (2-26), where $f=F^{\prime}$ and $F$ satisfies (1-9). Recalling that $\delta_{\varepsilon}=\varepsilon / \kappa_{1}$, where $\kappa_{1}$ is defined in (1-11), assume that there exist some $s_{1} \in\left(-T_{1}, T_{1}\right)$, $\rho_{1} \in\left(0, \rho_{0}\right)$, and $\zeta_{0} \geq \varepsilon^{2}$ such that

$$
\begin{array}{r}
\delta_{\varepsilon} \int_{\left\{s_{1}\right\} \times \mathbb{T}^{n} \times B_{v}\left(\rho_{1}\right)}\left(1+\left(y^{v}\right)^{2}\right) e_{\varepsilon}(v) d y^{\prime}-1 \leq \zeta_{0}, \\
\text { and } \mathscr{D}\left(v\left(s_{1}\right), \rho_{1} / 2\right) \leq \zeta_{0} .
\end{array}
$$

There exists a constant $C$, independent of $v$ and of $\varepsilon \in(0,1]$, such that

$$
\begin{array}{r}
\delta_{\varepsilon} \int_{\left\{s_{1}+s\right\} \times \mathbb{T}^{n} \times B_{v}\left(\rho_{1}-c_{*} s\right)}\left|D_{\tau} v\right|^{2}+\left(y^{v}\right)^{2}\left(\left|\nabla_{v} v\right|^{2}+\frac{1}{\varepsilon^{2}} F(v)\right) d y^{\prime} \leq C \zeta_{0}, \\
\delta_{\varepsilon} \int_{\left\{s_{1}+s\right\} \times \mathbb{T}^{n} \times B_{v}\left(\rho_{1}-c_{*} s\right)}\left(1+\left(y^{v}\right)^{2}\right) e_{\varepsilon}(v) d y^{\prime}-1 \leq C \zeta_{0}, \\
\text { and } \mathscr{D}\left(s_{1}+s ; \rho_{1} / 2\right) \leq C \zeta_{0},
\end{array}
$$

for all $s \in\left[0, \rho_{1} / 2 c_{*}\right]$ such that $s_{1}+s<T_{1}$.

Our first lemma will be needed to establish requirement (1-30), as discussed in the Introduction. In the statement and proof, we take all the $y^{\tau}$ variables to be frozen and consider a function $v$ of a single real variable $y^{v}$.

Lemma 11. Let $B_{v}(\rho):=(-\rho, \rho) \subset \mathbb{R}_{v}$ be an interval as above. There exists a constant $\kappa_{3}=\kappa_{3}(\rho)$ such that, if $v \in H^{1}\left(B_{v}(\rho)\right)$ and

then

$$
\mathscr{D}_{v}(v ; \rho) \leq \kappa_{3},
$$

$$
\delta_{\varepsilon} \int_{B_{v}(\rho)} e_{\varepsilon, v}(v) d y^{v} \geq 1-C e^{-C / \varepsilon} .
$$

Moreover, there exists a constant $\kappa_{4}=\kappa_{4}(\rho)$ such that, if (3-7) holds and

$$
\delta_{\varepsilon} \int_{B_{v}(\rho)} e_{\varepsilon, v}(v) d y^{v} \leq 1+\zeta_{0} \quad \text { for some } \zeta_{0} \in\left(0, \kappa_{4}\right)
$$

then

$$
\int_{B_{v}(\rho)}\left|\frac{1}{2} \varepsilon v_{y^{v}}^{2}-\frac{1}{\varepsilon} F(v)\right| d y^{v} \leq C\left(\sqrt{\zeta_{0}}+e^{-c / \varepsilon}\right) .
$$

The proof of Proposition 10 uses only the first conclusion (3-8) of this lemma. The other conclusion (3-10) is used in the proof of Theorem 22, when we deduce control over the full energy-momentum tensor from simpler energy estimates, like those of Proposition 10. 
Proof of Lemma 11. Step 1: Note that (3-8) is obvious if (3-9) fails, so it suffices to show that, if (3-7) and (3-9) hold, then both conclusions, (3-8) and (3-10), follow.

First, we define $Q(s):=\int_{0}^{s} \sqrt{2 F(\sigma)} d \sigma$ and, for any function $w \in H^{1}\left(B_{v}(\rho)\right)$, we estimate

$$
\varepsilon e_{\varepsilon, v}(w)=\frac{1}{2} \varepsilon w_{y^{v}}^{2}+\frac{1}{\varepsilon} F(w) \geq \sqrt{2 F(w)}\left|w_{y^{v}}\right|=\left|\partial_{y^{v}}(Q \circ w)\right| .
$$

Thus, since $\delta_{\varepsilon}=\varepsilon / \kappa_{1}$,

$$
\delta_{\varepsilon} \int_{B_{v}(\rho)} e_{\varepsilon, v}(w) \geq \frac{1}{\kappa_{1}} \int_{B_{v}(\rho)}\left|\partial_{y^{v}}(Q \circ w)\right|
$$

and, for any $w$, to obtain lower bounds for the left-hand side, it suffices to show that $y^{v} \mapsto Q\left(w\left(y^{v}\right)\right)$ has large total variation on $B_{v}(\rho)=(-\rho, \rho)$.

Step 2: Next, fix $\alpha>0$ so that $F^{\prime}=f$ is decreasing on $(1-\alpha, 1)$; this is possible as $F$ is $C^{2}$ and attains its minimum at 1 with $F^{\prime \prime}(1)>0$.

Let $v^{+}:=\sup _{y^{v} \in(\rho / 4,3 \rho / 4)} v\left(y^{v}\right)$. If $v^{+} \leq 1$, then (3-7) implies that

$$
\kappa_{3} \geq \int_{\rho / 4}^{3 \rho / 4} y^{\nu}\left|1-v\left(y^{v}\right)\right|^{2} d y^{\nu} \geq C \rho^{2}\left(1-v^{+}\right)^{2} .
$$

Thus, by choosing $\kappa_{3}$ small enough, we can arrange that $v^{+} \geq 1-\theta \alpha$ for some $\theta \in(0,1 / 2)$, to be chosen below. It then follows by the same argument that $v^{-}:=\inf _{y^{v} \in(-3 \rho / 4,-\rho / 4)} v\left(y^{v}\right) \leq-1+\theta \alpha$.

Step 3: We next claim that, once $\kappa_{3}$ and $\kappa_{4}$ are fixed in a suitable way, our hypotheses imply that

$$
v \geq 1-\alpha \text { in }(3 \rho / 4, \rho) \text { and } v \leq-1+\alpha \text { in }(-\rho,-3 \rho / 4) \text {. }
$$

This follows from (3-11) and Step 2 - the latter implies lower bounds on the total variation of $Q \circ w$ if (3-12) fails, and these lower bounds can be made to contradict (3-11) and (3-9).

In more detail, let us suppose (toward a contradiction) that the first inequality in (3-12) fails. Then, using Step 2, there exist points $y^{\nu, 1}<y^{\nu, 2}<y^{\nu, 3}$ such that $v\left(y^{\nu, 1}\right)<-1+\theta \alpha, v\left(y^{\nu, 2}\right)>1-\theta \alpha$, and $v\left(y^{\nu, 3}\right)<1-\alpha$. Hence, using the fact that $Q$ is nondecreasing (as the antiderivative of the positive function $\sqrt{2 F}$ ), we have (the first inequality following from (3-9) and (3-11))

$$
\begin{aligned}
\kappa_{1}\left(1+\kappa_{4}\right) & \geq \int_{B_{v}(\rho)}\left|\partial_{y^{v}}(Q \circ v)\right| \geq\left|\int_{y^{v, 1}}^{y^{v, 2}} \partial_{y^{v}}(Q \circ v) d y^{v}\right|+\left|\int_{y^{v, 2}}^{y^{v, 3}} \partial_{y^{v}}(Q \circ v) d y^{v}\right| \\
& \geq|Q(1-\theta \alpha)-Q(-1+\theta \alpha)|+|Q(1-\alpha)-Q(1-\theta \alpha)| \\
& \geq|Q(1-\theta \alpha)-Q(-1+\theta \alpha)|+2 \kappa_{1} \kappa_{4},
\end{aligned}
$$

where for the last step we chose $\kappa_{4}:=\left(2 \kappa_{1}\right)^{-1}|Q(1-\alpha)-Q(1-\alpha / 2)|$ (recall that $\left.\theta \leq \frac{1}{2}\right)$. This inequality is false when $\theta=0$, since $\kappa_{1}=Q(1)-Q(-1)$, and so it also fails for sufficiently small $\theta \in\left(0, \frac{1}{2}\right)$. Hence, we can choose $\kappa_{3}$ small enough to obtain a contradiction.

Step 4: We now replace $v$ on the interval $(3 \rho / 4, \rho)$ by the minimizer of the functional

$$
w \mapsto \int_{3 \rho / 4}^{\rho} e_{\varepsilon, v}(w) d y^{v}
$$


subject to the boundary conditions $w(3 \rho / 4)=v(3 \rho / 4)$ and $w(\rho)=v(\rho)$. Let $v_{1}$ denote the function obtained in this way. Standard maximum principle arguments ${ }^{6}$ imply that $v_{1}\left(\frac{7}{8} \rho\right) \geq 1-C e^{-c / \varepsilon}$. In a similar way, we can modify $v_{1}$ on $(-\rho,-3 \rho / 4)$ to obtain a function $v_{2}$ with less energy than that of $v_{1}$, and such that $v_{2}(-\rho)=v(-\rho)$, and $v_{2}(-7 \rho / 8) \leq-1+C e^{-c / \varepsilon}$.

Thus, $\left|Q\left(v_{2}\left(\frac{7}{8} \rho\right)\right)-Q(1)\right| \leq C e^{-c / \varepsilon}$ and similarly $\left|Q\left(v_{2}\left(-\frac{7}{8} \rho\right)\right)-Q(-1)\right| \leq C e^{-c / \varepsilon}$. As a result, using (3-9) and (3-11) as in Step 3, and recalling that $\kappa_{1}=Q(1)-Q(-1)$, we obtain

$$
\begin{aligned}
\kappa_{1}\left(1+\zeta_{0}\right) & \geq \int_{B_{v}(\rho)} \varepsilon e_{\varepsilon, v}(v) d y^{v} \geq \int_{B_{v}(\rho)} \varepsilon e_{\varepsilon, v}\left(v_{2}\right) d y^{v} \\
& \geq\left|Q\left(v_{2}(-\rho)\right)-Q\left(v_{2}\left(-\frac{7}{8} \rho\right)\right)\right|+\left|Q\left(v_{2}\left(-\frac{7}{8} \rho\right)\right)-Q\left(v_{2}\left(\frac{7}{8} \rho\right)\right)\right|+\left|Q\left(v_{2}\left(\frac{7}{8} \rho\right)\right)-Q\left(v_{2}(\rho)\right)\right| \\
& \geq\left|Q\left(v_{2}(-\rho)\right)-Q(-1)\right|+\kappa_{1}+\left|Q(1)-Q\left(v_{2}(\rho)\right)\right|-C e^{-c / \varepsilon} .
\end{aligned}
$$

This implies (3-8). Also, since $v_{2}=v$ at $\pm \rho$, the above implies that

$$
\begin{aligned}
Q(v(\rho))-Q(v(-\rho)) & =\kappa_{1}+Q(v(\rho))-Q(1)-(Q(v(-\rho))-Q(-1)) \\
& \geq \kappa_{1}-|Q(v(\rho))-Q(1)|-(Q(v(-\rho))-Q(-1)) \\
& \geq \kappa_{1}\left(1-\zeta_{0}\right)-C e^{-c / \varepsilon} .
\end{aligned}
$$

Step 5 We now use (3-13) to prove (3-10). First note that

$$
\begin{aligned}
\int_{B_{v}(\rho)}\left|\frac{1}{2} \varepsilon v_{y^{v}}^{2}-\frac{1}{\varepsilon} F(v)\right| d y^{v} & \leq \int_{B_{v}(\rho)}\left|\sqrt{\varepsilon} v_{y^{v}}-\frac{\sqrt{2 F(v)}}{\sqrt{\varepsilon}}\right|\left|\sqrt{\varepsilon} v_{y^{v}}+\frac{\sqrt{2 F(v)}}{\sqrt{\varepsilon}}\right| d y^{v} \\
& \leq C\left(\int_{B_{v}(\rho)}\left|\sqrt{\varepsilon} v_{y^{v}}-\frac{\sqrt{2 F(v)}}{\sqrt{\varepsilon}}\right|^{2} d y^{v}\right)^{1 / 2}\left(\int_{B_{v}(\rho)} \varepsilon e_{\varepsilon, v}(v) d y^{v}\right)^{1 / 2} .
\end{aligned}
$$

Expanding the square and recalling that $\sqrt{2 F}=Q^{\prime}$, we see that

$$
\begin{aligned}
\int_{B_{v}(\rho)} \frac{1}{2}\left|\sqrt{\varepsilon} v_{y^{v}}-\frac{\sqrt{2 F(v)}}{\sqrt{\varepsilon}}\right|^{2} d y^{v} & =\int_{B_{v}(\rho)} e_{\varepsilon, v}(v) d y^{v}-\int_{B_{v}(\rho)} Q^{\prime}(v) v_{y^{v}} d y^{v} \\
& =\int_{B_{v}(\rho)} e_{\varepsilon, v}(v) d y^{v}-(Q(v(\rho))-Q(v(-\rho))) \\
& \leq \kappa_{1}\left(1+\zeta_{0}\right)-\left(\kappa_{1}\left(1-\zeta_{0}\right)-C e^{-c / \varepsilon}\right) \quad(\text { using (3-9) and (3-13)) } \\
& \leq C \zeta_{0}+C e^{-c / \varepsilon} .
\end{aligned}
$$

Combining these inequalities and again appealing to (3-9), we arrive at (3-10).

The next lemma is used to establish requirement (1-32), as discussed in the Introduction. In this lemma we write $v$ as a function of two variables, $y^{0}$ and $y^{v}$.

${ }^{6}$ The point is that one can easily check that

$$
w\left(y^{\nu}\right):=1-\alpha \frac{\cosh \left(b\left(y^{\nu}-7 \rho / 8\right) / \varepsilon\right)}{\cosh (b \rho / 8 \varepsilon)}
$$

satisfies $-w^{\prime \prime}+\varepsilon^{-2} f(w) \leq 0$ in $(3 \rho / 4, \rho)$, if $b$ is fixed small enough (depending on $F$ ). Then, in view of (3-12) and the fact that $f$ is decreasing on $(1-\alpha, 1)$, one can use the maximum principle to find that $v_{1}>w$ in $(3 \rho / 4, \rho)$. 
Lemma 12. Let $B_{v}(\rho) \subset \mathbb{R}$ be an interval as above, and let $v \in H^{1}\left((0, \tau) \times B_{v}(\rho)\right)$ for some $\tau>0$. There exists a constant $C$, depending on $\rho$ but independent of $\tau$ and $\varepsilon \in(0,1]$, such that

$$
\int_{B_{v}(\rho)}\left|y^{\nu}\right|\left|v\left(0, y^{\nu}\right)-v\left(\tau, y^{\nu}\right)\right|^{2} d y^{\nu} \leq C \int_{(0, \tau) \times B_{\nu}(\rho)} \frac{1}{2} \varepsilon v_{y^{0}}^{2}+\frac{\left(y^{\nu}\right)^{2}}{\varepsilon} F(v) d y^{v} d y^{0} .
$$

Proof. For $Q: \mathbb{R} \rightarrow \mathbb{R}$ as above, such that $Q^{\prime}(s)=\sqrt{2 F(s)}$,

$$
\frac{1}{2} \varepsilon v_{y^{0}}^{2}+\frac{\left(y^{v}\right)^{2}}{\varepsilon} F(v) \geq\left|y^{v}\right| \sqrt{2 F(v)}\left|v_{y^{0}}\right|=\left|y^{v}\right|\left|Q(v)_{y^{0}}\right| .
$$

By integrating this inequality, we find that

$$
\begin{aligned}
\int_{(0, \tau) \times B_{v}(\rho)} \frac{1}{2} \varepsilon v_{y^{0}}^{2}+\frac{y^{2}}{\varepsilon} F(v) d y^{v} d y^{0} & \geq \int_{B_{v}(\rho)} \int_{0}^{\tau}\left|y^{v}\right|\left|Q(v)_{y^{0}}\right| d y^{0} d y^{v} \\
& \geq \int_{B_{v}(\rho)}\left|y^{\nu}\right|\left|Q\left(v\left(\tau, y^{\nu}\right)\right)-Q\left(v\left(0, y^{\nu}\right)\right)\right| d y^{v} .
\end{aligned}
$$

Finally, our assumption (1-9) that $F(s) \geq(1-|s|)^{2}$ and elementary calculus imply that

$$
|Q(b)-Q(a)| \geq c(b-a)^{2},
$$

and the lemma follows.

Proof of Proposition 10. Since the equation is well posed in $H^{1} \times L^{2}$, and since all the quantities in the statement are continuous in $H^{1} \times L^{2}$, we may prove the proposition for $v$ smooth.

In the proof we will write simply $\mathscr{D}(\cdot)$ instead of $\mathscr{D}\left(\cdot ; \rho_{1} / 2\right)$.

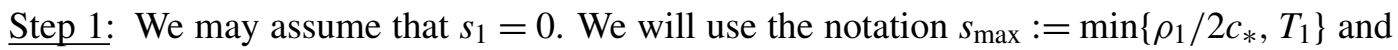

$$
W_{v}(s):=B_{v}\left(\rho_{1}-c_{*} s\right), \quad W(s):=\mathbb{T}^{n} \times W_{v}(s) .
$$

We define

$$
\begin{aligned}
& \zeta_{1}(s):=\delta_{\varepsilon} \int_{\{s\} \times W(s)}\left(1+\left(y^{v}\right)^{2}\right) e_{\varepsilon}(v) d y^{\prime}-1, \\
& \zeta_{2}(s):=\mathscr{D}(v(s)), \\
& \zeta_{3}(s):=\delta_{\varepsilon} \int_{\{s\} \times W(s)}\left|D_{\tau} v\right|^{2}+\left(y^{v}\right)^{2}\left(\left|\nabla_{v} v\right|^{2}+\frac{1}{\varepsilon^{2}} F(v)\right) d y^{\prime} .
\end{aligned}
$$

We first claim that

$$
\zeta_{1}(s) \leq C \zeta_{0}+C \int_{0}^{s} \zeta_{3}(\sigma) d \sigma \quad \text { for } s \in\left(0, s_{\max }\right]
$$

Towards this end, we compute

$$
\begin{aligned}
\zeta_{1}^{\prime}(s)=I_{1}-c_{*} I_{2}, \quad \text { where } I_{1} & :=\delta_{\varepsilon} \int_{\{s\} \times W(s)}\left(1+\left(y^{\nu}\right)^{2}\right) \frac{\partial}{\partial y^{0}} e_{\varepsilon}(v) d y^{\prime}, \\
I_{2} & :=\delta_{\varepsilon} \int_{\{s\} \times \mathbb{T}^{n} \times \partial W_{v}(s)}\left(1+\left(y^{\nu}\right)^{2}\right) e_{\varepsilon}(v) d y^{\tau^{\prime}} .
\end{aligned}
$$


To estimate $I_{1}$, we use Lemma 6 and integrate by parts in the spatial variables. From (2-28), we easily see that $\left|y^{v}\right|\left|\varphi^{v}\right| \leq C\left(\left|D_{\tau} v\right|^{2}+\left(y^{\nu}\right)^{2}\left|\nabla_{\nu} v\right|^{2}\right)$. Thus, we arrive at

$$
I_{1} \leq C \delta_{\varepsilon} \int_{\{s\} \times W(s)}\left(\left|D_{\tau} v\right|^{2}+\left(y^{\nu}\right)^{2}\left|\nabla_{\nu} v\right|^{2}\right) d y^{\prime}+\delta_{\varepsilon} \int_{\{s\} \times \mathbb{T}^{n} \times \partial W_{v}(s)}\left(1+\left(y^{\nu}\right)^{2}\right)\left|\varphi^{N}\right| d y^{\tau^{\prime}} .
$$

Our choice (3-4) of $c_{*}$ exactly guarantees that $\left|\varphi_{N}\right| \leq c_{*} e_{\varepsilon}(v)$, so that the boundary term above is dominated by $-c_{*} I_{2}$. It follows that $\zeta_{1}^{\prime} \leq C \zeta_{3}$. Since it is clear from (3-5) that $\zeta_{1}(0) \leq \zeta_{0}$, we conclude that (3-14) holds.

Step 2: Next, we estimate $\zeta_{2}$. Using the hypotheses and Lemma 12, we find that

$$
\begin{aligned}
\zeta_{2}(s) & \leq 2 \mathscr{D}(v(0))+2 \int_{\mathbb{T}^{n} \times B_{v}\left(\rho_{1} / 2\right)}\left|y^{v}\right|\left|v\left(s, y^{\prime}\right)-v\left(0, y^{\prime}\right)\right|^{2} d y^{\prime} \\
& \leq 2 \zeta_{0}+C \int_{\mathbb{T}^{n}}\left(\int_{0}^{s} \int_{B_{v}\left(\rho_{1} / 2\right)} \frac{1}{2} \varepsilon\left|v_{y^{0}}\right|^{2}+\frac{\left(y^{v}\right)^{2}}{\varepsilon} F(v) d y^{v} d y^{0}\right) d y^{\tau \prime} \\
& \leq 2 \zeta_{0}+C \int_{0}^{s} \zeta_{3}(\sigma) d \sigma
\end{aligned}
$$

for $s \leq s_{\max }$. We have changed the order of integration and used the fact that

$$
\mathbb{T}^{n} \times B_{v}\left(\rho_{1} / 2\right)=W_{v}\left(\rho_{1} / 2 c_{*}\right) \subset W_{v}(s) \text { for } s \leq s_{\max } \leq \rho_{1} / 2 c_{*} .
$$

Step 3: Finally, we claim that

$$
\zeta_{3}(s) \leq C\left(\zeta_{1}(s)+\zeta_{2}(s)+e^{-C / \varepsilon}\right)
$$

for every $s \in\left(0, s_{\max }\right]$. We fix such an $s$, and we often write $v(\cdot)$ instead of $v(s, \cdot)$. Note that $(2-25)$ implies that

$$
\left(1+\left(y^{v}\right)^{2}\right) e_{\varepsilon}(v) \geq \frac{1}{2} \lambda\left|D_{\tau} v\right|^{2}+\left(1+\left(y^{v}\right)^{2}\right) e_{\varepsilon, v}(v) .
$$

It follows from this and the definitions of $\zeta_{1}$ and $\zeta_{3}$ that

$$
\zeta_{1}(s) \geq c \zeta_{3}(s)+\delta_{\varepsilon} \int_{\{s\} \times W(s)} e_{\varepsilon, v}(v) d y^{\prime}-1 .
$$

Thus, it suffices to show that

$$
1-\delta_{\varepsilon} \int_{\{s\} \times W(s)} e_{\varepsilon, v}(v) d y^{\prime} \leq C \zeta_{2}(s)+C e^{-c / \varepsilon} .
$$

To do this, we say that a point $y^{\tau \prime} \in \mathbb{T}^{n}$ is good if

$$
\mathscr{D}_{v}\left(v\left(y^{\tau \prime}\right)\right) \leq \kappa_{3},
$$

and bad otherwise, for $v\left(y^{\tau \prime}\right)\left(y^{\nu}\right):=v\left(y^{\tau^{\prime}}, y^{\nu}\right)$. Then, Chebyshev's inequality implies that

$$
\mid\left\{y^{\tau^{\prime}} \in \mathbb{T}^{n}: y^{\tau \prime} \text { is bad }\right\} \mid \leq \frac{1}{\kappa_{3}} \int_{\{s\} \times \mathbb{T}^{n}} \mathscr{D}_{v}\left(v\left(y^{\tau^{\prime}}\right)\right) d y^{\tau^{\prime}}=C \mathscr{D}(v(s))=C \zeta_{2}(s) .
$$


Thus $\mid\left\{y^{\tau \prime} \in \mathbb{T}^{n}: y^{\tau \prime}\right.$ is good $\} \mid \geq 1-C \zeta_{2}(s)$ and, so, Lemma 11 implies that

$$
\begin{aligned}
\delta_{\varepsilon} \int_{\{s\} \times W(s)} e_{\varepsilon, v}(v) d y^{\prime} & \geq \int_{\left\{\left(s, y^{\tau}\right): y^{\left.\tau^{\prime} \in \mathbb{T}^{n} \text { is good }\right\}}\right.}\left(\delta_{\varepsilon} \int_{W_{v}(s)} e_{\varepsilon, v}(v) d y^{\nu}\right) d y^{\tau^{\prime}} \\
& \geq\left(1-C \zeta_{2}(s)\right)\left(1-C e^{-c / \varepsilon}\right) \quad \text { by using (3-8). }
\end{aligned}
$$

This proves (3-17), and hence (3-16).

Step 4: By combining the previous few steps and recalling that $\zeta_{0} \geq \varepsilon^{2}$, we see that

$$
\zeta_{3}(s) \leq C \zeta_{0}+C \int_{0}^{s} \zeta_{3}(\sigma) d \sigma
$$

so Gronwall's inequality implies that there exists some $C$ such that $\zeta_{3}(s) \leq C \zeta_{0}$ for all $s \in\left(0, \rho_{1} / 2 c_{*}\right)$. Then, (3-14) and (3-15) imply that $\zeta_{1}(s), \zeta_{2}(s) \leq C \zeta_{0}$. These estimates imply all the conclusions of the proposition.

\section{Initial energy estimates, $k=1$}

In this section, we indicate how to modify the above arguments to obtain control over $v$ on a portion of a hypersurface of the form $\left\{y^{0}=\right.$ constant $\}$, starting from our assumptions (2-31)-(2-36) about $u$ at $t=0$, which translate to information about $v$ on a hypersurface of the form $\left\{\left(b\left(y^{\prime}\right), y^{\prime}\right): y^{\prime} \in \mathbb{T}^{n} \times B_{v}\left(\rho_{0}\right)\right\}$, with $b$ in general a non-constant function. (Recall that the function $b$ was found in Lemma 8.) This is in general needed before we can start to iterate Proposition 10.

We note that if we assume that the minimal surface $\Gamma$ has velocity 0 at time $t=0$, then it is easy to check that $b\left(y^{\prime}\right) \equiv 0$. As a result, the hypotheses (3-5) and (3-6) of Proposition 10 follow immediately in this case from our general assumptions (2-31) and (2-34)-(2-36) on the initial data. So, the reader who is willing to accept this restriction on $\Gamma$ can skip this section (and Section 5.3) without any loss.

We continue to follow the notational conventions for the case $k=1$, summarized at the beginning of Section 3. We will prove:

Proposition 13. Assume that $v:\left(-T_{1}, T_{1}\right) \times \mathbb{T}^{n} \times B_{v}\left(\rho_{0}\right) \rightarrow \mathbb{R}$ is a solution of (2-26) with data that satisfies (2-34)-(2-36) on the hypersurface $\left\{\left(b\left(y^{\prime}\right), y^{\prime}\right): y^{\prime} \in \mathbb{T}^{n} \times B_{v}\left(\rho_{0}\right)\right\}$.

There exist $s_{1}>0$ and $\rho_{1}>0$ for which $v$ satisfies the hypotheses (3-5) and (3-6) of Proposition 10, with $\zeta_{0}$ replaced by $C \zeta_{0}$, and such that, in addition,

$$
\delta_{\varepsilon} \int_{\left\{y \in\left(-T_{1}, s_{1}\right) \times \mathbb{T}^{n} \times B_{v}\left(\rho_{1}\right): \psi^{0}(y)>0\right\}}\left(\left|D_{\tau} v\right|^{2}+\left|y^{v}\right|^{2}\left(\left|\nabla_{\nu} v\right|^{2}+\frac{1}{\varepsilon^{2}} F(v)\right)\right) d y \leq C \zeta_{0} .
$$

If we simply tried to repeat our earlier arguments, we would have to worry about the way in which a cone with slope $c_{*}$ intersects the initial hypersurface, and these considerations would force us to impose unnatural restrictions on the initial velocity of the surface $\Gamma$. We, therefore, exploit finite propagation speed in a different and sharper way than in our earlier arguments. (We could have done this earlier, but we wanted to present our basic estimate in a relatively simple setting.) This, and other considerations, forces us to introduce a certain amount of notation.

We start by defining

$$
\mathscr{C}:=\left\{(t, x) \in \mathbb{R}^{1+N}: \operatorname{dist}\left(x, \Gamma_{0}\right)<\tau-t \text { and } t>0\right\} .
$$


where dist denotes the Euclidean distance function, $\Gamma_{0}=\left\{H\left(0, y^{\tau^{\prime}}\right): y^{\tau^{\prime}} \in \mathbb{T}^{n}\right\}$, and $\tau>0$ is chosen so small that

$$
\mathscr{b} \Subset \operatorname{Image}(\psi) .
$$

Note that $\mathscr{C}$ consists of the set of points for which the solution of the semilinear wave equation (1-1) depends solely on the data in the set $\mathscr{C}_{0}:=\left\{x \in \mathbb{R}^{N}: \operatorname{dist}\left(x, \Gamma_{0}\right)<\tau\right\}$. We continue by defining

$$
\begin{aligned}
& V:=\psi^{-1}(\mathscr{C}), \\
& s_{0}:=\inf \left\{y^{0} \in\left(-T_{1}, T_{1}\right):\left(\left\{y^{0}\right\} \times \mathbb{T}^{n} \times B_{v}\left(\rho_{0}\right)\right) \cap V \neq \varnothing\right\}, \text { and } \\
& V^{*}:=\left\{y=\left(y^{0}, y^{\prime}\right) \in\left(s_{0}, T_{1}\right) \times \mathbb{T}^{n} \times B_{v}\left(\rho_{0}\right):\left(s, y^{\prime}\right) \in V \text { for some } s \geq y^{0}\right\}
\end{aligned}
$$

Thus $V^{*}$ is just $V$ "extended downward" in the timelike $y^{0}$ variable, to $s_{0}$. For $s \in R$, we define

$$
V(s):=\left\{y \in V: y^{0}<s\right\} \quad \text { and } \quad V^{*}(s):=\left\{y \in V^{*}: y^{0}<s\right\} .
$$

We further define

$$
\begin{aligned}
& \partial_{0} V(s):=\left\{y \in \partial V(s): \psi^{0}(y)=0\right\}, \\
& \partial_{1} V(s):=\left\{y=\left(y^{0}, y^{\prime}\right) \in \partial V(s): y^{0}=s\right\}, \\
& \partial_{2} V(s):=\partial V(s) \backslash\left(\partial_{0} V(s) \cup \partial_{1} V(s)\right) .
\end{aligned}
$$

We will also write

$$
\begin{aligned}
& \partial_{1} V^{*}(s):=\left\{y=\left(y^{0}, y^{\prime}\right) \in \partial V^{*}(s): y^{0}=s\right\} \\
& \partial_{0} V:=\left\{y \in \partial V: \psi^{0}(y)=0\right\} \\
& W_{0}:=\left\{y^{\prime} \in \mathbb{T}^{n} \times B_{v}\left(\rho_{0}\right):\left(y^{0}, y^{\prime}\right) \in \partial_{0} V \text { for some } y^{0}\right\} .
\end{aligned}
$$

Finally, for $i=0,1,2$, we define

$$
W_{i}(s):=\left\{y^{\prime} \in \mathbb{T}^{n} \times B_{v}\left(\rho_{0}\right):\left(y^{0}, y^{\prime}\right) \in \partial_{i} V(s) \text { for some } y^{0}\right\}
$$

and similarly $W_{i}^{*}(s)$.

The next lemma collects some geometric facts that we will need about the sets defined above.

Lemma 14. We have

$$
\left(W_{0}(s) \backslash W_{1}(s)\right) \cap W_{1}^{*}(s)=\varnothing \quad \text { for all } s .
$$

In addition, there exist $s_{1}>0$ and $\rho_{1}>0$ such that

$$
\left(s_{0}, s_{1}\right) \times \mathbb{T}^{n} \times B_{v}\left(\rho_{1}\right) \subset V^{*} \quad \text { and } \quad\left\{s_{1}\right\} \times \mathbb{T}^{n} \times B_{v}\left(\rho_{1}\right) \subset V .
$$

Proof. To prove (4-3), fix $y^{\prime} \in W_{0}(s) \backslash W_{1}(s)$. The definitions imply that the line $\left\{\left(y^{0}, y^{\prime}\right): y^{0} \in \mathbb{R}\right\}$ intersects $\partial_{0} V(s)$ and does not meet $\partial_{1} V(s)$, so it must leave $\bar{V}$ at a point $\left(\sigma, y^{\prime}\right)$ with $\sigma<s$. Arguments like those of Lemma 8 show that once the line has left $\bar{V}$, it cannot re-enter, since, if it did, the timelike curve $s \mapsto X(s):=\psi\left(s, y^{\prime}\right)$ (see Lemma 8) would intersect $\partial^{+} \mathscr{C}:=\{(t, x) \in \partial \mathscr{b}: t>0\}$ more than once, which is impossible. Thus, the line does not intersect $\bar{V}^{*}$ at any point $\left(y^{0}, y^{\prime}\right)$ with $y^{\prime}>\sigma$ and, so, it cannot intersect $\partial_{1} V^{*}(s) \subset\left\{\left(y^{0}, s\right) \in \bar{V}^{*}: y^{0}=s\right\}$. Thus, $y^{\prime} \notin W_{1}^{*}(s)$, proving (4-3). 
Next, the existence of $s_{1}, \rho_{1}>0$ satisfying (4-4) follows from the fact that the (Euclidean) distance from $\{0\} \times \mathbb{T}^{n} \times\{0\}=\psi^{-1}\left(\Gamma_{0}\right)$ to $\partial^{+} V:=\partial V \backslash \partial_{0} V=\psi^{-1}\left(\partial^{+} \mathscr{C}\right)$ is positive. This last fact, in turn, is clear from the fact that the distance from $\Gamma_{0}$ to $\partial^{+} \mathscr{C}$ is positive, together with the smoothness of $\psi$.

Recall that $v_{0}: \mathbb{T}^{n} \times B_{v}\left(\rho_{0}\right) \cong\{0\} \times \mathbb{T}^{n} \times B_{v}\left(\rho_{0}\right)$ was defined in (2-33). We extend $v_{0}$ to $\left(-T_{1}, T_{1}\right) \times$ $\mathbb{T}^{n} \times B_{v}(\rho)$ in such a way that it is independent of $y^{0}$; this extended function is still denoted by $v_{0}$.

The remainder of this section contains the proof of Proposition 13. In the proof, when we want to distinguish between row vectors and column vectors (which one can think as vectors and covectors, respectively), we will write $\bar{\xi}$ to denote a column vector, with components $\xi^{\alpha}$, and $\underline{\xi}$ for a row vector, with components $\xi_{\alpha}$.

Proof of Proposition 13. As in Proposition 10, it suffices to prove the statement for $v$ smooth in $\bar{V}$.

Step 1: We define $v^{*}: V^{*} \rightarrow \mathbb{R}$ by

$$
v^{*}(y)= \begin{cases}v(y) & \text { if } y \in V \\ v_{0}(y) & \text { if } y \in V^{*} \backslash V .\end{cases}
$$

Since $v=v_{0}$ on $\bar{V} \cap\left(V^{*} \backslash V\right)=\partial_{0} V$, it is easy to see that $v^{*}$ is Lipschitz in $V^{*}$. Note, however, that the derivatives of $v^{*}$ are in general discontinuous across $\partial_{0} V$.

We define

$$
\begin{aligned}
& \zeta_{1}(s):=\delta_{\varepsilon} \int_{\partial_{1} V^{*}(s)}\left(1+\left(y^{v}\right)^{2}\right) e_{\varepsilon}\left(v^{*}\right) d y^{\prime}-1, \\
& \zeta_{2}(s):=\mathscr{D}\left(v^{*}(s) ; \rho_{1} / 2\right), \\
& \zeta_{3}(s):=\delta_{\varepsilon} \int_{\partial_{1} V^{*}(s)}\left(\left|D_{\tau} v^{*}\right|^{2}+\left(y^{\nu}\right)^{2} e_{\varepsilon, v}\left(v^{*}\right)\right) d y^{\prime} .
\end{aligned}
$$

In view of (4-4), we can repeat word for word the arguments from the proof of Proposition 10, to find

$$
\begin{aligned}
& \zeta_{3}(s) \leq C\left(\zeta_{1}(s)+\zeta_{2}(s)+e^{-c / \varepsilon}\right), \\
& \zeta_{2}(s) \leq 2 \zeta_{2}\left(s_{0}\right)+C \int_{s_{0}}^{s} \zeta_{3}(\sigma) d \sigma,
\end{aligned}
$$

for every $s \in\left[s_{0}, s_{1}\right]$. Also, the definition of $s_{0}$ implies that $v^{*}=v_{0}$ on $\partial_{1} V^{*}\left(s_{0}\right):=\left\{s_{0}\right\} \times W_{0}$, so that $\zeta_{2}\left(s_{0}\right) \leq \zeta_{0}$ by $(2-36)$. Thus,

for every $s \in\left[s_{0}, s_{1}\right]$.

$$
\zeta_{2}(s) \leq C \zeta_{0}+C \int_{s_{0}}^{s} \zeta_{3}(\sigma) d \sigma
$$

The remainder of the proof is devoted to the estimate of $\zeta_{1}$. Since $v^{*}$ is smooth away from $\partial_{0} V$ and (by Fubini's Theorem) $\partial_{1} V^{*}(s) \cap \partial_{0} V$ has $\mathscr{H}^{N}$ measure 0 for $\mathscr{L}^{1}$-a.e. $s$, the definition of $v^{*}$ implies that

$$
e_{\varepsilon}\left(v^{*}\right)= \begin{cases}e_{\varepsilon}(v) & \mathscr{H}^{N} \text {-a.e. in } \partial_{1} V(s) \\ e_{\varepsilon}\left(v_{0}\right) & \mathscr{H}^{N} \text {-a.e. in } \partial_{1} V^{*}(s) \backslash \partial_{1} V(s)\end{cases}
$$

for a.e. $s$. Also, if $[\cdots]$ denotes an integrand that does not depend on the $y^{0}$ variable, then clearly $\int_{\partial_{1}^{*} V(s) \backslash \partial_{1} V(s)}[\cdots] d y^{\prime}=\int_{W_{1}^{*}(s) \backslash W(s)}[\cdots] d y^{\prime}$. Thus, for a.e. $s$,

$$
\int_{\partial_{1} V^{*}(s)}\left(1+\left(y^{\nu}\right)^{2}\right) e_{\varepsilon}\left(v^{*}\right) d y^{\prime}=\int_{\partial_{1} V(s)}\left(1+\left(y^{\nu}\right)^{2}\right) e_{\varepsilon}(v) d y^{\prime}+\int_{W_{1}^{*}(s) \backslash W_{1}(s)}\left(1+\left(y^{\nu}\right)^{2}\right) e_{\varepsilon}\left(v_{0}\right) d y^{\prime}
$$


Step 2: We claim that, for a.e. $s$,

$$
\begin{aligned}
\delta_{\varepsilon} \int_{\partial_{1} V(s)}\left(1+\left(y^{v}\right)^{2}\right) e_{\varepsilon}(v) & d y^{\prime} \\
\leq & \delta_{\varepsilon} \int_{\partial_{0} V(s)}\left(1+\left(y^{v}\right)^{2}\right)\left(-n_{0} e_{\varepsilon}(v)+n_{i} \varphi^{i}\right) d \mathscr{H}^{N}(d y)+C \int_{s_{0}}^{s} \zeta_{3}(\sigma) d \sigma,
\end{aligned}
$$

where $\underline{n}(y)$ denotes the (Euclidean) outer unit normal at a point $y \in \partial V(s)$, thought of as a row vector with components $n_{\alpha}$, and $\varphi^{i}$ is defined in (2-28) and appears in the local energy estimate of Lemma 6.

Step 2.1: To prove (4-11), we will first integrate by parts and show that some of the boundary terms have a sign, and hence can be discarded. (In this, we basically follow the proof of Proposition 10.) For this, it is useful to define $\widetilde{\mathscr{T}}_{\varepsilon}=\widetilde{\mathscr{T}}_{\varepsilon}(v)$ by

$$
\widetilde{\mathscr{T}}_{\varepsilon, \beta}^{\alpha}:=\delta_{\beta}^{\alpha}\left(\frac{1}{2} g^{\gamma \delta} v_{y^{\gamma}} v_{y^{\delta}}+\frac{1}{\varepsilon^{2}} F(v)\right)-g^{\alpha \gamma} v_{y^{\gamma}} v_{y^{\beta}} .
$$

Observe, from the definitions, that ${ }^{7}$

$$
\widetilde{\mathscr{T}}_{\varepsilon, 0}^{0}(v)=e_{\varepsilon}(v) \quad \text { and } \quad \tilde{\mathscr{T}}_{\varepsilon, 0}^{i}(v)=-\varphi^{i},
$$

so that the conclusion of Lemma 6 can be written $\partial_{y^{\alpha}} \widetilde{\mathscr{T}}_{\varepsilon, 0}^{\alpha} \leq C\left(\left|D_{\tau} v\right|^{2}+\left(y^{\nu}\right)^{2}\left|\nabla_{\nu} v\right|^{2}\right)$.

We now compute

$$
\begin{aligned}
\delta_{\varepsilon} \int_{V(s)} \partial_{y^{\alpha}}\left(\left(1+\left(y^{\nu}\right)^{2}\right) \widetilde{\mathscr{T}}_{\varepsilon, 0}^{\alpha}\right) d y & \leq C \delta_{\varepsilon} \int_{V(s)}\left(\left(\left|D_{\tau} v\right|^{2}+\left(y^{\nu}\right)^{2}\left|\nabla_{\nu} v\right|^{2}\right)+y^{N} \widetilde{\mathscr{T}}_{\varepsilon, 0}^{N}\right) d y \\
& \leq C \delta_{\varepsilon} \int_{V(s)}\left(\left|D_{\tau} v\right|^{2}+\left(y^{\nu}\right)^{2}\left|\nabla_{\nu} v\right|^{2}\right) d y \\
& \leq C \int_{s_{0}}^{s} \zeta_{3}(\sigma) d \sigma .
\end{aligned}
$$

On the other hand, we can integrate by parts to rewrite the left-hand side as an integral over $\partial V(s)$. Then, noting that $\underline{n}(y)=(1,0, \ldots, 0)$ for $y \in \partial_{1} V(s)$, we find that

$$
\begin{aligned}
& \delta_{\varepsilon} \int_{V(s)} \partial_{y^{\alpha}}\left(\left(1+\left(y^{\nu}\right)^{2}\right) \widetilde{\mathscr{T}}_{\varepsilon, 0}^{\alpha}\right)=\delta_{\varepsilon} \int_{\partial_{1} V(s)}\left(1+\left(y^{\nu}\right)^{2}\right) e_{\varepsilon}(v) d y^{\prime}+\delta_{\varepsilon} \int_{\partial_{0} V(s)}\left(\left(1+\left(y^{\nu}\right)^{2}\right) n_{\alpha} \widetilde{\mathscr{T}}_{\varepsilon, 0}^{\alpha}\right) d \mathscr{H}^{N}(y) \\
& +\delta_{\varepsilon} \int_{\partial_{2} V(s)}\left(\left(1+\left(y^{v}\right)^{2}\right) n_{\alpha} \tilde{\mathscr{T}}_{\varepsilon, 0}^{\alpha}\right) d \mathscr{H}^{N}(y) \text {. }
\end{aligned}
$$

By combining this with (4-14) and recalling (4-13), we see that our claim (4-11) will follow if we can show that the last integral on the right-hand side is positive.

Step 2.2: To do this, we will show that

$$
n_{\alpha}(y) \widetilde{\mathscr{T}}_{\varepsilon, 0}^{\alpha}(y) \geq 0 \quad \text { for a.e. } y \in \partial_{2} V(s) .
$$

We first check that

$$
g^{\alpha \beta} n_{\alpha} n_{\beta}=0 \text { for a.e. } y \in \partial_{2} V \text {. }
$$

\footnotetext{
${ }^{7}$ In fact, $\widetilde{T}_{\varepsilon}$ is just the energy-momentum tensor for $u$, expressed in terms of the $y$ coordinates. The fact that, when written in the $y$ coordinates, the energy-momentum tensor is divergence-free, takes the form $\partial_{y^{\alpha}}\left(\widetilde{\mathscr{T}}_{\varepsilon, \beta}^{\alpha}(v) \sqrt{-g}\right)=0$ for all $\beta$.
} 
In fact, we will show that this holds at every $y \in \partial_{2} V$ such that $\partial C$ has a tangent plane at $x=\psi(y)$; this is a set of full measure. Fix such a $y$ and let $\bar{w}=\left(w^{\alpha}\right)$ be any (column) vector tangent to $\partial \mathscr{C}$ at $x$. Also, let $\underline{m}(x)$ denote the (Euclidean) outer unit normal to $\mathscr{b}$ at $x \in \partial \mathscr{C}$, again thought of as a row vector with components $m_{\alpha}$. Writing $\varphi=\psi^{-1}$ as usual, since $\varphi$ maps $\partial \mathscr{C}$ to $\partial V$, it is clear that $D \varphi(x) \bar{w}$ is tangent to $\partial V$ at $\varphi(x)=y$, which implies that $\underline{n}(y) D \varphi(x) \bar{w}=0$. Since this holds for all tangent vectors $\bar{w}$ at $x$, it follows that $\underline{n}(y) D \varphi(x)$ is parallel to the Euclidean unit normal $\underline{m}$ to $\partial C$ at $x$; that is, $\underline{n}(y) D \varphi(x)=\lambda \underline{m}(x)$ for some $\lambda \in \mathbb{R}$. The form of $\mathscr{C}$ implies that $\underline{m}$ is a null vector, so that

$$
0=\lambda^{2} \eta^{\alpha \beta} m_{\alpha} m_{\beta}=\lambda^{2} \eta^{\alpha \beta} n_{\gamma} \varphi_{\alpha}^{\gamma} m_{\delta} \varphi_{\beta}^{\delta}=g^{\gamma \delta} n_{\gamma} n_{\delta},
$$

proving (4-16). Note also that $n_{0}(y)>0$ for $y \in \partial_{2} V$, and recall further that $F(u) \geq 0$. Thus,

$$
\begin{aligned}
n_{\alpha} \widetilde{T}_{\varepsilon, 0}^{\alpha} & =\frac{n_{0}}{\varepsilon^{2}} F(u)+\frac{n_{0}}{2} g^{\alpha \beta} v_{y^{\alpha}} v_{y^{\beta}}-v_{y^{0}} g^{\alpha \beta} n_{\alpha} v_{y^{\beta}} \\
& \geq \frac{n_{0}}{2} g^{\alpha \beta} v_{y^{\alpha}} v_{y^{\beta}}-v_{y^{0}} g^{\alpha \beta} n_{\alpha} v_{y^{\beta}}=\frac{n_{0}}{2} g^{\alpha \beta}\left(D v-\frac{v_{y^{0}}}{n_{0}} n\right)_{\alpha}\left(D v-\frac{v_{y^{0}}}{n_{0}} n\right)_{\beta},
\end{aligned}
$$

using (4-16). If we write $\xi:=D v-\frac{v_{y^{0}}}{n_{0}} n$, then clearly $\xi_{0}=0$, which implies that

$$
g^{\alpha \beta} \xi_{\alpha} \xi_{\beta}=g^{i j} \xi_{i} \xi_{j}=a^{\alpha \beta} \xi_{\alpha} \xi_{\beta} \geq 0 .
$$

Thus, we have proved (4-15).

Step 3: Next, we note that

$$
-\int_{\partial_{0} V(s)}\left(1+\left(y^{\nu}\right)^{2}\right) n_{0}(y) e_{\varepsilon}(v)(y) d \mathscr{H}^{N}(d y)=\int_{W_{0}(s)}\left(1+\left(y^{\nu}\right)^{2}\right) e_{\varepsilon}(v)\left(b\left(y^{\prime}\right), y^{\prime}\right) d y,
$$

where we recall that $\partial_{0} V=\left\{\left(b\left(y^{\prime}\right), y^{\prime}\right): y^{\prime} \in W_{0}\right\}$, and hence that $\partial_{0} V(s)=\left\{\left(b\left(y^{\prime}\right), y^{\prime}\right): y^{\prime} \in W_{0}(s)\right\}$. This is obvious, because the Euclidean outer unit normal to $V(s)$ is given by $\underline{n}=(-1, \nabla b) /\left(1+|\nabla b|^{2}\right)^{1 / 2}$, with the minus sign appearing because $V$ sits above the graph. Thus, $-n_{0}\left(b\left(y^{\prime}\right), y^{\prime}\right)=\left(1+\left|\nabla b\left(y^{\prime}\right)\right|^{2}\right)^{-1 / 2}$, and then (4-17) follows from a change of variables using the area formula.

Step 4: Now, we combine (4-17) with (4-10) and (4-11), to find that

for a.e. $s \in\left[s_{0}, s_{1}\right]$, where

$$
\zeta_{1}(s) \leq C \int_{s_{0}}^{s} \zeta_{3}(\sigma) d \sigma+A+B
$$

$$
\begin{aligned}
& A:=\delta_{\varepsilon} \int_{W_{0}(s)}\left(1+\left(y^{\nu}\right)^{2}\right)\left(e_{\varepsilon}(v)-e_{\varepsilon}\left(v_{0}\right)\right)\left(b\left(y^{\prime}\right), y^{\prime}\right) d y^{\prime}+\delta_{\varepsilon} \int_{\partial_{0} V(s)}\left(1+\left(y^{\nu}\right)^{2}\right) n_{i} \varphi^{i} d \mathcal{H}^{N}, \\
& B:=\delta_{\varepsilon} \int_{W_{1}^{*}(s) \backslash W_{1}(s)}\left(1+\left(y^{\nu}\right)^{2}\right) e_{\varepsilon}\left(v_{0}\right) d y^{\prime}+\delta_{\varepsilon} \int_{W_{0}(s)}\left(1+\left(y^{\nu}\right)^{2}\right) e_{\varepsilon}\left(v_{0}\right) d y^{\prime}-1 .
\end{aligned}
$$

We have checked in Lemma 14 that $\left(W_{1}^{*}(s) \backslash W_{1}(s)\right) \cap W_{0}(s)=\varnothing$; this is equivalent to (4-3). Thus,

$$
B \leq \delta_{\varepsilon} \int_{W_{0}}\left(1+\left(y^{\nu}\right)^{2}\right) e_{\varepsilon}\left(v_{0}\right) d y^{\prime}-1 \leq \zeta_{0},
$$

by (2-34). To estimate $A$, we differentiate the identity $v\left(b\left(y^{\prime}\right), y^{\prime}\right)=v_{0}\left(y^{\prime}\right)$ to find that $v_{y^{0}} \nabla b+\nabla v=\nabla v_{0}$. Thus, $\left|D\left(v-v_{0}\right)\right|=\left|v_{y^{0}}(1,-\nabla b)\right| \leq C\left|v_{y^{0}}\right|$ at points $\left(b\left(y^{\prime}\right), y^{\prime}\right) \in \partial_{0} V$, using the control over $\|\nabla b\|_{\infty}$ 
obtained in Lemma 8. It follows that, at such points,

$$
e_{\varepsilon}(v)-e_{\varepsilon}\left(v_{0}\right)=\frac{1}{2} a^{\alpha \beta}\left(v-v_{0}\right)_{y^{\alpha}}\left(v+v_{0}\right)_{y^{\beta}} \leq C\left(v_{y^{0}}^{2}+\left|D_{\tau} v_{0}\right|^{2}+\left|v_{y^{0}}\right|\left|\nabla_{\nu} v_{0}\right|\right)
$$

Similarly, using (2-19), we see that $\left|\varphi^{i}\right| \leq C\left(v_{y^{0}}^{2}+\left|D_{\tau} v_{0}\right|^{2}+\left(y^{\nu}\right)^{2}\left|\nabla_{\nu} v_{0}\right|^{2}\right)$, so

$$
A \leq C \delta_{\varepsilon} \int_{\partial_{0} V}\left(v_{y^{0}}^{2}+\left|v_{y^{0}}\right|\left|\nabla_{\nu} v_{0}\right|\right) d \mathscr{H}^{N}+C \delta_{\varepsilon} \int_{W_{0}}\left(\left|D_{\tau} v_{0}\right|^{2}+\left(y^{\nu}\right)^{2}\left|\nabla_{\nu} v_{0}\right|^{2}\right) d y^{\prime} .
$$

Also, since $v_{0}\left(y^{\prime}\right)=v^{*}\left(s_{0}, y^{\prime}\right)$, we have

$$
\int_{W_{0}} \varepsilon\left(\left|D_{\tau} v_{0}\right|^{2}+\left(y^{\nu}\right)^{2}\left|\nabla_{\nu} v_{0}\right|^{2}\right) d y^{\prime} \leq \zeta_{3}\left(s_{0}\right) \leq C\left(\zeta_{1}\left(s_{0}\right)+\zeta_{2}\left(s_{0}\right)+e^{-c / \varepsilon}\right) \leq C \zeta_{0}
$$

here we used (4-7) for the second inequality, and (2-34) and (2-35) for the last. Using this and (2-35), we conclude that $A \leq C \zeta_{0}$ and, hence, that

$$
\zeta_{1}(s) \leq C \int_{s_{0}}^{s} \zeta_{3}(\sigma) d \sigma+C \zeta_{0}
$$

Step 5: The rest of the proof follows exactly that of Proposition 10. In the end, we find that $\zeta_{i}\left(s_{1}\right) \leq C \zeta_{0}$ for $i=1,2,3$ and, in view of (4-4), these estimates immediately imply the conclusion.

\section{Energy estimates, $k=2$}

In this section, we prove energy estimates like those from Sections 3 and 4 , but now in the case $k=2$, so that we consider a vector-valued function $v:\left(-T_{1}, T_{1}\right) \times \mathbb{T}^{n} \times B_{v}(\rho) \rightarrow \mathbb{R}^{2}$ solving (2-26), where $B_{v}(\rho) \subset \mathbb{R}_{v}^{2}$ now denotes a 2-dimensional ball, $\kappa_{2}$ is the constant chosen in $(2-23), \delta_{\varepsilon}=(\pi|\ln \varepsilon|)^{-1}$, and the nonlinearity in (1-1) is $f=\nabla F$, with $F: \mathbb{R}^{2} \rightarrow[0, \infty)$ satisfying (1-19).

The main results and proofs in this section are strictly analogous to Propositions 10 and 13 . The chief difference is that the "defect-confinement functional" $\mathscr{D}$ (discussed in the Introduction) has quite a different form than in the case $k=1$. Thus, the arguments we need in order to verify that the desired properties (1-30) and (1-32) hold, are quite different from (and more delicate than) their counterparts in the scalar case. Once suitable forms of these facts are established, we follow our earlier proofs with only cosmetic changes.

We will use machinery that relates the Jacobian and the Ginzburg-Landau energy. We will give precise statements of the facts we need from the literature, in the hope of rendering our arguments somewhat accessible to people who are not familiar with these results; see also the book [Sandier and Serfaty 2007] for a general reference on these topics. The results we use (see Lemmas 18, 19 and 21) are proved for $F_{\text {model }}(u)=\frac{1}{4}\left(|u|^{2}-1\right)^{2}$ in the sources we cite, but it is evident ${ }^{8}$ from the proofs that they still apply to functions $F$ satisfying the assumptions (1-19) that we impose here.

\footnotetext{
${ }^{8}$ In all the proofs we will cite, easy truncation arguments are used to reduce to, for example, the case of $u$ with $|u| \leq M$ a.e. for $M=2$; then, (1-19) implies that $\left(1 /(C \varepsilon)^{2}\right) F_{\text {model }}(u) \leq\left(1 / \varepsilon^{2}\right) F(u) \leq\left(1 /(\varepsilon / C)^{2}\right) F_{\text {model }}(u)$. It is then clear that results established for $F_{\text {model }}$ carry over to energy functionals that instead contain $F$, since everything we use is essentially unaffected if $\varepsilon$ is replaced by $C \varepsilon$ or $\varepsilon / C$.
} 
For $v \in H^{1}\left(\mathbb{T}^{n} \times B_{v}(\rho) ; \mathbb{R}^{2}\right)$ we take $\mathscr{D}$ to have the form (as when $\left.k=1\right)$

$$
\mathscr{D}(v ; \rho):=\int_{\mathbb{T}^{n}} \mathscr{D}_{v}\left(v\left(y^{\tau \prime}\right) ; \rho\right) d y^{\tau \prime},
$$

where $v\left(y^{\tau \prime}\right)\left(y^{v}\right)=v\left(y^{\tau \prime}, y^{v}\right)$. For $w=\left(w^{1}, w^{2}\right) \in H^{1}\left(B_{v}(\rho) ; \mathbb{R}^{2}\right)$, we define

$$
\mathscr{D}_{v}(w ; \rho):=\left\|J_{\nu} w-\pi \delta_{0}\right\| \|_{\rho}
$$

where, for a measure $\mu$ on $B_{v}(\rho)$,

$$
\|\| \mu \|_{\rho}:=\sup \left\{\int \omega\left(y^{v}\right) f\left(y^{v}\right) d y^{v}: \omega \in C_{c}^{2}\left(B_{\rho}\right),\left|\nabla \omega\left(y^{v}\right)\right| \leq\left|y^{v}\right|^{2},\|\omega\|_{W^{2, \infty}} \leq 1\right\} .
$$

(Clearly, ||$\cdot \mid \|_{\rho}$ also makes sense for some distributions that are less regular than measures, but we will not need that here.) Here, we are using the notation $J_{v} w=\operatorname{det} \nabla_{\nu} w$. We will also write $\boldsymbol{J}_{v} w$ for the 2-form $\boldsymbol{J}_{v} w=J_{v} w d y^{v}$, where $d y^{v}:=d y^{v, 1} \wedge d y^{v, 2}$. Note that

$$
J_{\nu} w:=d_{\nu} w^{1} \wedge d_{\nu} w^{2}, \quad \text { where } d_{\nu} w^{i}=\frac{\partial w^{i}}{\partial y^{\nu, 1}} d y^{\nu, 1}+\frac{\partial w^{i}}{\partial y^{\nu, 2}} d y^{\nu, 2} .
$$

(Recall that $y^{\nu, i}=y^{n+i}$.)

General results and heuristics about Jacobians and vortices (see, for example, [Sandier and Serfaty 2007]), together with the definition of the \|\|$\cdot\|\|_{\rho}$ norm, suggest that, if $w: B_{v}(\rho) \rightarrow \mathbb{R}^{2}$ is a function possessing a single "vortex of degree 1" localized near some point in $B_{v}(\rho / 2)$, then, roughly speaking,

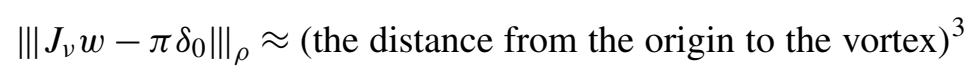

(The cubic scaling on the right-hand side is related to the condition $\left|\nabla \omega\left(y^{v}\right)\right| \leq\left|y^{v}\right|^{2}$ imposed on test functions, in the definition of $\||\cdot|\|_{\rho}$.) Thus, the right-hand side of (5-1) is the average of the above quantity over the tangential $y^{\tau}$ variables.

The first main result of this section parallels Proposition 10 above:

Proposition 15. Let $v:\left(-T_{1}, T_{1}\right) \times \mathbb{T}^{n} \times B_{v}\left(\rho_{0}\right) \rightarrow \mathbb{R}^{2}$ satisfy (2-26), where $B_{v}(\rho) \subset \mathbb{R}_{v}^{2}$ and $f=\nabla F$, with $F: \mathbb{R}^{2} \rightarrow \mathbb{R}$ satisfying (1-19). Recalling that $\delta_{\varepsilon}=(\pi \ln \varepsilon \mid)^{-1}$, assume that there exist $s_{1} \in\left(-T_{1}, T_{1}\right)$, $\rho_{1} \in\left(0, \rho_{0}\right)$, and $\zeta_{0} \geq \delta_{\varepsilon}$ such that

$$
\begin{gathered}
\delta_{\varepsilon} \int_{\left\{s_{1}\right\} \times \mathbb{T}^{n} \times B_{v}\left(\rho_{1}\right)}\left(1+\kappa_{2}\left|y^{v}\right|^{2}\right) e_{\varepsilon}(v) d y^{\prime}-1 \leq \zeta_{0} \quad \text { and } \\
\mathscr{D}\left(v(0) ; \rho_{1} / 2\right) \leq \zeta_{0} .
\end{gathered}
$$

There exists a constant $C$ such that

$$
\begin{array}{r}
\delta_{\varepsilon} \int_{\left\{s_{1}+s\right\} \times \mathbb{T}^{n} \times B_{v}\left(\rho_{1}-c_{*} s\right)}\left(\left|D_{\tau} v\right|^{2}+\left|y^{v}\right|^{2}\left(\left|\nabla_{\nu} v\right|^{2}+\frac{1}{\varepsilon^{2}} F(v)\right)\right) d y^{\prime} \leq C \zeta_{0}, \\
\delta_{\varepsilon} \int_{\left\{s_{1}+s\right\} \times \mathbb{T}^{n} \times B_{v}\left(\rho_{1}-c_{*} s\right)} e_{\varepsilon}(v)\left(1+\kappa_{2}\left|y^{v}\right|^{2}\right) d y^{\prime}-1 \leq C \zeta_{0}, \\
\text { and } \mathscr{D}\left(v(s) ; \rho_{1} / 2\right) \leq C \zeta_{0},
\end{array}
$$

for all $s \in\left[0, \rho_{1} / 2 c_{*}\right]$ such that $s_{1}+s<T_{1}$. Here, $c_{*}$ is as defined in (3-4). 
As remarked earlier, there does not exist any initial data satisfying (5-4) and (5-5) with $\zeta_{0} \ll \delta_{\varepsilon}$ when $k=2$, so the condition $\zeta_{0} \geq \delta_{\varepsilon}$ is not restrictive.

The second main result of this section parallels Proposition 13:

Proposition 16. Assume that $v:\left(-T_{1}, T_{1}\right) \times \mathbb{T}^{n} \times B_{v}\left(\rho_{0}\right) \rightarrow \mathbb{R}^{2}$ is a solution of (2-26), with data that satisfies (2-34)-(2-36) on the hypersurface $\left\{\left(b\left(y^{\prime}\right), y^{\prime}\right): y^{\prime} \in \mathbb{T}^{n} \times B_{v}\left(\rho_{0}\right)\right\}$, with $\zeta_{0} \geq \delta_{\varepsilon}$ and $\mathscr{D}$ as defined in (5-1).

There exist some $s_{1}>0$ and $\rho_{1}>0$ for which $v$ satisfies the hypotheses (5-4), (5-5) of Proposition 15, with $\zeta_{0}$ replaced by $C \zeta_{0}$, and such that, in addition,

$$
\delta_{\varepsilon} \int_{\left\{y \in\left(-T_{1}, s_{1}\right) \times \mathbb{T}^{n} \times B_{\nu}\left(\rho_{1}\right): \psi^{0}(y)>0\right\}}\left(\left|D_{\tau} v\right|^{2}+\left|y^{v}\right|^{2}\left(\left|\nabla_{\nu} v\right|^{2}+\frac{1}{\varepsilon^{2}} F(v)\right)\right) d y \leq C \zeta_{0} .
$$

5.1. Variational-stability estimates. We start by establishing some properties relating the $\|\mid \cdot\| \|_{\rho}$ norm of the Jacobian $J v$ and the Ginzburg-Landau energy $e_{\varepsilon, v}(v)$. These will be used to show that $\mathscr{D}(\cdot)$ satisfies the requirements (1-30) and (1-32) from the Introduction.

Our first result is analogous to Lemma 11, and establishes a form of (1-30). It is a straightforward consequence of the Jacobian machinery mentioned above.

Proposition 17. For $\rho>0$, there exist constants $\kappa_{3}$ and $C$, both depending on $\rho$, such that, if $w \in$ $H^{1}\left(B_{v}(\rho) ; \mathbb{R}^{2}\right)$ and

then

$$
\mathscr{D}_{v}(w ; \rho)=\left\|\mid J_{v} w-\pi \delta_{0}\right\|_{\rho} \leq \kappa_{3},
$$

$$
|\ln \varepsilon|^{-1} \int_{B} e_{\varepsilon, v}(w) d y^{\nu} \geq \pi-|\ln \varepsilon|^{-1} C .
$$

The proof of Proposition 17 uses the following facts:

Lemma 18. If $\varepsilon \in(0,1], w \in H^{1}\left(B_{v}(\rho) ; \mathbb{R}^{2}\right)$, and

then

$$
\left\|J_{v} w-\pi \delta_{0}\right\|_{W^{-1,1}\left(B_{v}(\rho)\right)} \leq \frac{\rho}{10}
$$

$$
\frac{1}{|\ln \varepsilon|} \int_{B_{v}(\rho)} e_{\varepsilon, v}(w) d y^{v} \geq \pi-\frac{C}{|\ln \varepsilon|} .
$$

This follows, for example, from a much sharper estimate proved in [Jerrard and Spirn 2007, Theorem 1.3]. A slightly different norm is used there in place of the $W^{-1,1}$ norm, but that result is easily seen to imply the one stated here.

Lemma 19. Suppose that $\varepsilon \in(0,1]$ and that $w \in H^{1}\left(B_{v}(\rho) ; \mathbb{R}^{2}\right)$ satisfies

$$
\frac{1}{|\ln \varepsilon|} \int_{B} e_{\varepsilon, v}(w) d y^{v} \leq 3 \pi / 2 \text {. }
$$

There exists an integer $\ell \in\{0, \pm 1\}$ and a point $\xi \in B$ such that

$$
\left\|J_{v} w-\pi \ell \delta_{\xi}\right\|_{W^{-1,1}\left(B_{v}(\rho)\right)} \leq C|\ln \varepsilon| \varepsilon^{1 / 4} .
$$

This follows from [Jerrard and Spirn 2007, Theorem 1.1]. 
Proof of Proposition 17. Fix $w \in H^{1}\left(B_{v}(\rho) ; \mathbb{R}^{2}\right)$. We may assume that

$$
\frac{1}{|\ln \varepsilon|} \int_{B_{v}(\rho)} e_{\varepsilon, v}(w) d y^{v} \leq 3 \pi / 2,
$$

since otherwise (5-7) is immediate. So, in view of Lemma 18, it suffices to show that there exists a constant $\kappa_{3}(\rho)$ such that, if (5-8) holds and $\left\|J_{v} w-\pi \delta_{0}\right\|_{\rho}<\kappa_{3}$, then

$$
\left\|J_{v} w-\pi \delta_{0}\right\|_{W^{-1,1}\left(B_{v}(\rho)\right)} \leq \frac{\rho}{10} .
$$

In fact, it suffices to show that there exists some $\varepsilon_{0}>0$ such that the above conclusion holds if $\varepsilon \in\left(0, \varepsilon_{0}\right)$ in (5-8), since we can arrange that (5-7) holds for $\varepsilon>\varepsilon_{0}$ by choosing $C$ large enough.

Now, (5-8) and Lemma 19 imply that there exist an integer $\ell$ with $|\ell| \leq 1$, and a point $\xi \in B_{v}(\rho)$ such that $\left\|J_{v} w-\pi \ell \delta_{\xi}\right\|_{W^{-1,1}\left(B_{v}(\rho)\right)} \leq C|\ln \varepsilon| \varepsilon^{1 / 4}$. Fix a function $\omega_{*} \in C_{c}^{2}(B)$, with $\left|\nabla \omega_{*}(y)\right| \leq|y|^{2}$ and $\left\|\omega_{*}\right\|_{W^{2, \infty}} \leq 1$, and such that $\omega_{*}(y)<\omega_{*}(0)$ if $y \neq 0$. Then, (5-6) and the definition of the $\|\mid \cdot\| \|_{\rho}$ norm imply that

$$
\int \omega_{*} J_{v} w d y^{\nu}-\pi \omega_{*}(0) \geq-\kappa_{3}
$$

On the other hand, the estimate $\left\|J_{\nu} w-\pi \ell \delta_{\xi}\right\|_{W^{-1,1}(B)} \leq C|\ln \varepsilon| \varepsilon^{1 / 4}$ implies that

$$
\int \omega_{*} J_{v} w d y^{v}-\pi \ell \omega_{*}(\xi) \leq C\left\|\omega_{*}\right\|_{W^{1, \infty}}|\ln \varepsilon| \varepsilon^{1 / 4} \leq C|\ln \varepsilon| \varepsilon^{1 / 4}
$$

Thus,

$$
\ell \omega_{*}(\xi) \geq \omega_{*}(0)-\frac{\kappa_{3}}{\pi}-C|\ln \varepsilon| \varepsilon^{1 / 4} .
$$

Since $\omega_{*}(0)>0$, this implies that $\ell=1$ for all sufficiently small $\varepsilon>0$, if $\kappa_{3}$ is fixed small enough. Then, $\left\|J w(\tau)-\pi \delta_{\xi}\right\|_{W^{-1,1}\left(B_{v}(\rho)\right)} \leq C|\ln \varepsilon| \varepsilon^{1 / 4}$ and, as a result,

$$
\begin{aligned}
\left\|J(w(\tau))-\pi \delta_{0}\right\|_{W^{-1,1}\left(B_{v}(\rho)\right)} & \leq C|\ln \varepsilon| \varepsilon^{1 / 4}+\pi\left\|\delta_{\xi}-\delta_{0}\right\|_{W^{-1,1}\left(B_{v}(\rho)\right)} \\
& \leq C|\ln \varepsilon| \varepsilon^{1 / 4}+\pi|\xi|,
\end{aligned}
$$

where the last inequality follows immediately from the definition of the $W^{-1,1}$ norm. Since $\omega_{*}$ is continuous and achieves its maximum exactly at the origin, (5-10) implies that, fixing $\kappa_{3}$ still smaller if necessary, $\pi|\xi|<\rho / 20$ and, as a result, (5-9) holds for all small $\varepsilon$.

The second result about the \|\|$\cdot \|_{\rho}$ norm is analogous to Lemma 11, and establishes a form of the requirement (1-32); in fact, the norm is designed exactly so that an estimate of the form (5-11) holds. In the lemma, we write $v$ as a function of $\left(y^{0}, y^{\nu}\right) \in \mathbb{R} \times \mathbb{R}_{v}^{2}$.

Proposition 20. Let $v \in H^{1}\left((0, \tau) \times B_{v}(\rho) ; \mathbb{R}^{2}\right)$ for some $\rho, \tau>0$. There exist positive constants $C$ and $\alpha$, depending on $\rho$ but independent of $\tau$ and $\varepsilon \in(0,1]$, such that

$$
\begin{aligned}
&\|\mid\| J_{v} v(\tau, \cdot)-J_{v} v(0, \cdot)\|\|_{\rho} \leq C \delta_{\varepsilon} \int_{(0, \tau) \times B_{v}(\rho)}\left(\left|y^{v}\right|^{2}+\varepsilon^{\alpha}\right)\left(\frac{1}{2}|D v|^{2}+\frac{1}{\varepsilon^{2}} F(v)\right) d y^{v} d y^{0} \\
& \quad+C \varepsilon^{\alpha}\left(1+\int_{\{0\} \times B_{v}(\rho)} e_{\varepsilon, v}(v) d y^{v}+\int_{\{\tau\} \times B_{v}(\rho)} e_{\varepsilon, v}(v) d y^{v}\right) .
\end{aligned}
$$


We believe that the $\varepsilon^{\alpha}$ in the first integral on the right-hand side of (5-11) could be removed with some work, but the estimate is false without the boundary terms in the second line of (5-11). In any case, all these terms will be negligible in our later arguments.

The proof of Proposition 20 requires the following:

Lemma 21. There exist universal constants $C, \alpha>0$ such that, given any $U \subset \mathbb{R}^{3}=\mathbb{R}_{y^{0}} \times \mathbb{R}_{v}^{2}$ and $w \in H^{1}\left(U ; \mathbb{R}^{2}\right)$,

$$
\begin{aligned}
\left|\int_{U} \omega \wedge \boldsymbol{J} w\right| \leq \frac{C}{|\ln \varepsilon|} \int_{U}|\omega|\left(\frac{1}{2}|D w|^{2}+\frac{1}{\varepsilon^{2}} F(w)\right) \\
\quad+C \varepsilon^{\alpha}\left(1+\|D \omega\|_{\infty}\right)\left(1+\|\omega\|_{\infty}+\int_{U}(1+|\omega|)\left(\frac{1}{2}|D w|^{2}+\frac{1}{\varepsilon^{2}} F(w)\right)\right)
\end{aligned}
$$

for every compactly supported Lipschitz continuous 1-form $\omega$ in $U$, and every $\varepsilon \in(0,1]$. Here, $\boldsymbol{J} w$ denotes the 2-form $d w^{1} \wedge d w^{2}=\left(w_{y^{0}}^{1} d y^{0}+d_{\nu} w^{1}\right) \wedge\left(w_{y^{0}}^{2} d y^{0}+d_{\nu} w^{2}\right)$.

This is [Jerrard 2007, Lemma 9], with notation adapted to our setting. In (5-12), Dw denotes, as usual, the gradient in all three variables.

Proof of Proposition 20.

Step 1: Fix $v \in H^{1}\left((0, \tau) \times B_{v}(\rho) ; \mathbb{R}^{2}\right)$. In order to prove (5-11), we must estimate

$$
\int_{B_{v}(\rho)} \omega\left(J_{v} v\left(\tau, y^{v}\right)-J_{v} v\left(0, y^{v}\right)\right) d y^{v}
$$

for an arbitrary $\omega \in C_{c}^{\infty}\left(B_{v}(\rho)\right)$ such that $|\nabla \omega(y)| \leq|y|^{2}$ and $\|\omega\|_{W^{2, \infty}} \leq 1$. We fix such a test function $\omega$, and we start by rewriting the previous expression. For this, let $\delta$ denote a positive number to be fixed later (not to be confused with $\delta_{\varepsilon}$ ), and define $V:(-\delta, \tau+\delta) \times B_{v}(\rho) \rightarrow \mathbb{R}^{2}$ by

$$
V\left(y^{0}, y^{\nu}\right)= \begin{cases}v\left(0, y^{\nu}\right) & \text { if }-\delta<y^{0} \leq 0, \\ v\left(y^{0}, y^{v}\right) & \text { if } 0 \leq y^{0} \leq \tau, \\ v\left(\tau, y^{v}\right) & \text { if } \tau \leq y^{0} \leq \tau+\delta .\end{cases}
$$

Let $\chi \in C_{c}^{\infty}(-\delta, \tau+\delta)$ be a function such that

$$
\chi\left(y^{0}\right) \equiv 1 \text { for } y^{0} \in[0, \tau] \text { and }\left\|\chi^{\prime}\right\|_{\infty} \leq C\left(1+\delta^{-1}\right) .
$$

Since $J_{v} V\left(y^{0}\right)=J_{v} v(0)$ for $y^{0} \in(-\delta, 0]$, and $J_{v} V\left(y^{0}\right)=J_{v} v(\tau)$ for $y^{0} \in[\tau, \tau+\delta)$,

$$
\begin{aligned}
\int_{B_{v}(\rho)} \omega\left(J_{\nu} v\left(\tau, y^{\nu}\right)-J_{\nu} v\left(0, y^{\nu}\right)\right) d y^{\nu} & =-\int_{-\delta}^{\tau+\delta} \chi^{\prime}\left(y^{0}\right)\left(\int_{B_{v}(\rho)} \omega\left(y^{\nu}\right) J_{v} V d y^{\nu}\right) d y^{0} \\
& =-\int_{(-\delta, \tau+\delta) \times B_{v}(\rho)}\left(\omega\left(y^{\nu}\right) \chi^{\prime}\left(y^{0}\right) d y^{0}\right) \wedge \boldsymbol{J} V .
\end{aligned}
$$

We continue by observing that

$$
\omega\left(y^{\nu}\right) \chi^{\prime}\left(y^{0}\right) d y^{0}=\omega\left(y^{\nu}\right) d \chi\left(y^{0}\right)=d\left(\omega\left(y^{\nu}\right) \chi\left(y^{0}\right)\right)-\chi\left(y^{0}\right) d \omega\left(y^{\nu}\right) .
$$


Also, since $\boldsymbol{J} V=d\left(V^{1} \wedge d V^{2}\right)$, it is clear that $d(\boldsymbol{J} V)=0$, so that $d(\chi \omega) \wedge \boldsymbol{J} V=d(\chi \omega \wedge \boldsymbol{J} V)$ and, thus, the right-hand side of (5-13) can be rewritten

$$
\begin{aligned}
-\int_{(-\delta, \tau+\delta) \times B_{v}(\rho)}\left(\omega \chi^{\prime} d y^{0}\right) \wedge \boldsymbol{J} V & =\int_{(-\delta, \tau+\delta) \times B_{v}(\rho)} \chi d \omega \wedge \boldsymbol{J} V-\int_{(-\delta, \tau+\delta) \times B_{v}(\rho)} d(\chi \omega) \wedge \boldsymbol{J} V \\
& =\int_{(-\delta, \tau+\delta) \times B_{v}(\rho)} \chi d \omega \wedge \boldsymbol{J} V
\end{aligned}
$$

Step 2: The properties of $\omega$ and the choice of $\chi$ imply that

$$
|\chi d \omega(y)| \leq\left|y^{v}\right|^{2} \text { and } \quad\|D(\chi d \omega)\|_{\infty} \leq C \delta^{-1} .
$$

It thus follows from Lemma 21 that

$$
\begin{aligned}
\left|\int_{(-\delta, \tau+\delta) \times B_{v}(\rho)} \chi d \omega \wedge \boldsymbol{J} V\right| \leq C|\ln \varepsilon|^{-1} \int_{(-\delta, \tau+\delta) \times B_{v}(\rho)}\left|y^{\nu}\right|^{2}\left(\frac{1}{2}|D V|^{2}+\frac{1}{\varepsilon^{2}} F(V)\right) d y^{\nu} d y^{0} \\
+C \varepsilon^{\alpha}\left(1+\delta^{-1}\right)\left(1+\int_{(-\delta, \tau+\delta) \times B_{v}(\rho)}\left(\frac{1}{2}|D V|^{2}+\frac{1}{\varepsilon^{2}} F(V)\right) d y^{v} d y^{0}\right) .
\end{aligned}
$$

We now fix $\delta:=\varepsilon^{\alpha / 2}$ and recall the definition of $V$, to find that

$$
\begin{aligned}
\left|\int_{(-\delta, \tau+\delta) \times B_{v}(\rho)} \chi d \omega \wedge \boldsymbol{J} V\right| \leq C|\ln \varepsilon|^{-1} \int_{(0, \tau) \times B_{v}(\rho)}\left(\left|y^{\nu}\right|^{2}+\varepsilon^{\alpha / 2}\right)\left(\frac{1}{2}\left|v_{y^{0}}\right|^{2}+e_{\varepsilon, v}(v)\right) d y^{v} d y^{0} & \\
& +C \varepsilon^{\alpha / 2}\left(1+\int_{\{0\} \times B_{v}(\rho)} e_{\varepsilon, v}(v) d y^{v}+\int_{\{\tau\} \times B_{v}(\rho)} e_{\varepsilon, v}(v) d y^{\nu}\right) .
\end{aligned}
$$

The conclusion now follows by recalling (5-13) and (5-14), and renaming $\alpha$.

5.2. Proof of Proposition 15. As in Proposition 10 it suffices to consider smooth solutions $v$.

To simplify we will write $\mathscr{D}(\cdot)$ and $\||\cdot|||$ instead of $\mathscr{D}\left(\cdot ; \rho_{1} / 2\right)$ and $\||\cdot|\|_{\rho_{1} / 2}$.

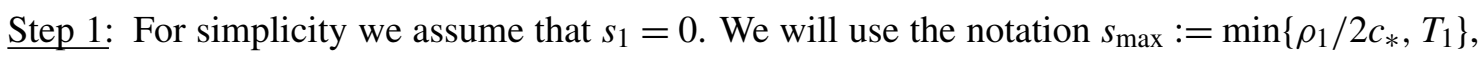

$$
W_{v}(s):=B_{v}\left(\rho_{1}-c_{*} s\right) \quad \text { and } \quad W(s):=\mathbb{T}^{n} \times W_{v}(s) .
$$

We define

$$
\begin{aligned}
& \zeta_{1}(s):=\delta_{\varepsilon} \int_{\{s\} \times W(s)}\left(1+\kappa_{2}\left|y^{v}\right|^{2}\right) e_{\varepsilon}(v) d y^{\prime}-1, \\
& \zeta_{2}(s):=\mathscr{D}(v(s)), \\
& \zeta_{3}(s):=\delta_{\varepsilon} \int_{\{s\} \times W(s)}\left(\left|D_{\tau} v\right|^{2}+\left|y^{v}\right|^{2} e_{\varepsilon, v}(v)\right) d y^{\prime} .
\end{aligned}
$$

(Recall that $\kappa_{2}$ was fixed in $(2-23)$, and that we took $\kappa_{2}=1$ for $k=1$.) We first claim that

$$
\zeta_{1}(s) \leq \zeta_{0}+C \int_{0}^{s} \zeta_{3}(\sigma) d \sigma \quad \text { for } 0<s \leq s_{\max } .
$$


Indeed, exactly as before, we compute that $\zeta_{1}^{\prime}(s)=I_{1}-c_{*} I_{2}$, where

$$
\begin{aligned}
& I_{1}:=\delta_{\varepsilon} \int_{\{s\} \times W(s)}\left(1+\kappa_{2}\left|y^{\nu}\right|^{2}\right) \frac{\partial}{\partial y^{0}} e_{\varepsilon}(v) d y^{\prime} \\
& I_{2}=\delta_{\varepsilon} \int_{\{s\} \times \mathbb{T}^{n} \times \partial W_{v}(s)}\left(1+\kappa_{2}\left|y^{\nu}\right|^{2}\right) e_{\varepsilon}(v) d \mathscr{H}^{N-1}\left(y^{\prime}\right) .
\end{aligned}
$$

And, exactly as before, in $I_{1}$ we use the differential inequality (2-27) satisfied by the energy, and integrate by parts in the spatial variables. As before, our choice (3-4) of $c_{*}$ guarantees that the boundary term that arises, involving an integral over $\{s\} \times \mathbb{T}^{n} \times \partial W_{v}(s)$, is dominated by $-c_{*} I_{2}$. This leads, as before, to the differential inequality

$$
\zeta_{1}^{\prime} \leq C \zeta_{3}
$$

Since our assumption (5-4) states exactly that $\zeta_{1}(0) \leq \zeta_{0}$, we conclude that (5-15) holds.

Step 2: We estimate $\zeta_{2}$. It is clear that $\||\cdot|||$ is a norm, so

$$
\mathscr{D}_{v}\left(v\left(s, y^{\tau^{\prime}}\right)\right) \leq \mathscr{D}_{v}\left(v\left(0, y^{\tau^{\prime}}\right)\right)+\left\|J_{v} v\left(s, y^{\tau^{\prime}}\right)-J_{v} v\left(0, y^{\tau^{\prime}}\right)\right\|
$$

for every $\left(s, y^{\tau \prime}\right)$, by the triangle inequality. It follows that

$$
\begin{aligned}
& \zeta_{2}(s) \leq \mathscr{D}(v(0))+\int_{\mathbb{T}^{n}}\left\|J_{v} v\left(0, y^{\tau^{\prime}}\right)-J_{v} v\left(s, y^{\tau^{\prime}}\right)\right\| d y^{\tau^{\prime}} \\
& \stackrel{(5-5),(5-11)}{\leq} \zeta_{0}+C \delta_{\varepsilon} \int_{\mathbb{T}^{n}} \int_{(0, s) \times B_{v}\left(\rho_{1} / 2\right)}\left|D_{\tau} v\right|^{2}+\left(\left|y^{\nu}\right|^{2}+\varepsilon^{\alpha}\right) e_{\varepsilon, v}(v) d y^{v} d y^{0} d y^{\tau^{\prime}} \\
& +C \varepsilon^{\alpha}+C \varepsilon^{\alpha} \int_{\mathbb{T}^{n}}\left(\int_{\{0\} \times B_{v}\left(\rho_{1} / 2\right)} e_{\varepsilon, v}(v) d y^{v}+\int_{\{s\} \times B_{v}\left(\rho_{1} / 2\right)} e_{\varepsilon, v}(v) d y^{v}\right) d y^{\tau^{\prime}} .
\end{aligned}
$$

Also, since $B_{v}\left(\rho_{1} / 2\right) \subset W_{v}(s)$ for every $s \leq \rho_{0} / 2 c_{*}$, the definitions yield

$$
\int_{\mathbb{T}^{n}} \int_{\{s\} \times B_{v}\left(\rho_{1} / 2\right)} e_{\varepsilon}(v) d y^{v} d y^{\tau \prime} \leq C \delta_{\varepsilon}^{-1}\left(\zeta_{1}(s)+1\right) \leq C|\ln \varepsilon|\left(\zeta_{1}(s)+1\right),
$$

and similarly for $s=0$. By combining these and rearranging, we find that if $0 \leq s \leq s_{\max }$, then

$$
\zeta_{2}(s) \leq \zeta_{0}+C \int_{0}^{s}\left(\zeta_{3}(\sigma)+\varepsilon^{\alpha}\left(\zeta_{1}(\sigma)+C\right)\right) d \sigma+C \varepsilon^{\alpha}+C \varepsilon^{\alpha / 2}\left(\zeta_{0}+\zeta_{1}(s)+C\right) .
$$

Step 3: Finally, we show (by exactly the same arguments as in the corresponding step of the proof of Proposition 10) that

$$
\zeta_{3}(s) \leq C\left(\zeta_{1}(s)+\zeta_{2}(s)+|\ln \varepsilon|^{-1}\right)
$$

for every $s \in\left[0, s_{\max }\right]$. We fix such an $s$, and we write $v(\cdot)$ instead of $v(s, \cdot)$. It follows, from the definitions of $\zeta_{1}, \zeta_{3}$ and the choice (2-23) of $\kappa_{2}$, that

$$
\zeta_{1}(s) \geq c \zeta_{3}(s)+\delta_{\varepsilon} \int_{\{s\} \times W\left(\rho_{1} / 2 c_{*}\right)} e_{\varepsilon, v}(v) d y^{\prime}-1 .
$$

We say that a point $y^{\tau^{\prime}} \in \mathbb{T}^{n}$ is good if $\mathscr{D}_{v}\left(v\left(y^{\tau \prime}\right)\right) \leq \kappa_{3}$, and bad otherwise. Then, Chebyshev's inequality and (5-1) imply that $\mid\left\{y^{\tau \prime} \in \mathbb{T}^{n}: y^{\tau \prime}\right.$ is good $\} \mid \geq 1-C \zeta_{2}(s)$ and, exactly as in (3-19), but appealing to 
Proposition 17 instead of Lemma 11, we infer that

$$
\delta_{\varepsilon} \int_{\{s\} \times W\left(\rho_{1} / 2 c_{*}\right)} e_{\varepsilon, v}(v) d y^{\prime} \geq\left(1-C \zeta_{2}(s)\right)\left(1-C|\ln \varepsilon|^{-1}\right) .
$$

Combining this inequality with (5-18), we obtain (5-17).

Step 4: By combining the previous few steps, we see that

$$
\zeta_{3}(s) \leq C \zeta_{0}+C|\ln \varepsilon|^{-1}+C \int_{0}^{s} \zeta_{3}(\sigma) d \sigma+C \varepsilon^{\alpha} \int_{0}^{s} \int_{0}^{\sigma} \zeta_{3}(t) d t d \sigma .
$$

If we define $\zeta_{4}(s):=\zeta_{3}(s)+\zeta_{0}+|\ln \varepsilon|^{-1}+\varepsilon^{\alpha} \int_{0}^{s} \zeta_{3}(\sigma) d \sigma$, it follows (since $\zeta_{0} \geq \delta_{\varepsilon}$ ) that

$$
\zeta_{4}(s) \leq C \int_{0}^{s} \zeta_{4}(\sigma) d \sigma \quad \forall s \in\left[0, s_{\max }\right], \quad \zeta_{4}(0) \leq C \zeta_{0} .
$$

Gronwall's inequality then implies that $\zeta_{4}(s) \leq C \zeta_{0}$ for all $s \in\left[0, s_{\max }\right]$. The conclusions of the proposition follow from this, together with (5-15) and (5-16).

5.3. Proof of Proposition 16. Finally, we present the proof of Proposition 16. We use notation from Section 4, such as $V^{*}(s), \partial_{i} V^{*}(s)$ and so on.

As usual, we may assume by an approximation argument, relying on standard well-posedness theory for (2-26), that $v$ is smooth on $\bar{V}$. Define $v^{*}$ as in (4-5), and set

$$
\begin{aligned}
& \zeta_{1}(s)=\delta_{\varepsilon} \int_{\partial_{1} V^{*}(s)}\left(1+\kappa_{2}\left|y^{v}\right|^{2} \text { bigr }\right) e_{\varepsilon}\left(v^{*}\right) d y^{\prime}-1, \\
& \zeta_{2}(s)=\mathscr{D}\left(v^{*}(s) ; \rho_{1} / 2\right), \\
& \zeta_{3}(s)=\delta_{\varepsilon} \int_{\partial_{1} V^{*}(s)}\left(\left|D_{\tau} v^{*}\right|^{2}+\left|y^{v}\right|^{2} e_{\varepsilon, v}\left(v^{*}\right)\right) d y^{\prime} .
\end{aligned}
$$

We repeat exactly the arguments of Proposition 15, to find that

$$
\zeta_{2}(s) \leq C \int_{s_{0}}^{s} \zeta_{3}(\sigma)+\varepsilon^{\alpha}\left(\zeta_{1}(\sigma)+C\right) d \sigma+C \varepsilon^{\alpha}+C \varepsilon^{\alpha / 2}\left(\zeta_{0}+\zeta_{1}(s)+C\right)
$$

and

$$
\zeta_{3}(s) \leq C\left(\zeta_{1}(s)+\zeta_{2}(s)+|\ln \varepsilon|^{-1}\right) .
$$

To estimate $\zeta_{1}$, we argue as in the proof of Proposition 13; that is, we apply the divergence theorem to

where

$$
\int_{V(s)} \partial_{y^{\alpha}}\left(\left(1+\kappa_{2}\left|y^{v}\right|^{2}\right) \widetilde{T}_{\varepsilon, 0}^{\alpha}\right)
$$

$$
\widetilde{T}_{\varepsilon, \beta}^{\alpha}(y):=\delta_{\beta}^{\alpha}\left(\frac{1}{2} \varepsilon g^{\gamma \delta} v_{y^{\gamma}} \cdot v_{y^{\delta}}+\frac{1}{\varepsilon} F(v)\right)-\varepsilon g^{\alpha \gamma} v_{y^{\gamma}} \cdot v_{y^{\beta}},
$$

and we rewrite, noting that $n_{\alpha}(y) \widetilde{T}_{\varepsilon, 0}^{\alpha}(y) \geq 0$ for a.e. $y \in \partial_{2} V(s)$, exactly as before. This eventually yields, for a.e. $s \in\left[s_{0}, s_{1}\right]$,

$$
\zeta_{1}(s) \leq C \int_{s_{0}}^{s} \zeta_{3}(\sigma) d \sigma+A+B
$$


where

$$
\begin{aligned}
A & :=\delta_{\varepsilon} \int_{W_{0}(s)}\left(1+\kappa_{2}\left|y^{v}\right|^{2}\right)\left(e_{\varepsilon}(v)-e_{\varepsilon}\left(v_{0}\right)\right)\left(b\left(y^{\prime}\right), y^{\prime}\right) d y^{\prime}+\delta_{\varepsilon} \int_{\partial_{0} V(s)}\left(1+\kappa_{2}\left|y^{v}\right|^{2}\right) n_{i} \varphi^{i} d \mathscr{H}^{N}, \\
B & :=\delta_{\varepsilon} \int_{\left(W_{1}^{*}(s) \backslash W_{1}(s)\right) \cup W_{0}(s)}\left(1+\kappa_{2}\left|y^{v}\right|^{2}\right) e_{\varepsilon}\left(v_{0}\right) d y^{\prime}-1 .
\end{aligned}
$$

We proceed exactly as in the proof of Proposition 13, using Lemmas 8 and 14, the hypotheses (2-34)(2-36), and elementary arguments, to show that $A \leq C \zeta_{0}$ and $B \leq C \zeta_{0}$ for a.e. $s \in\left[s_{0}, s_{1}\right]$ and, hence, that $\zeta_{1} \leq C \int_{s_{0}}^{s} \zeta_{3}(\sigma) d \sigma+C \zeta_{0}$.

The proof is now finished, exactly as in Step 4 of the proof of Proposition 15.

\section{Proof of Theorems 1 and 2}

We combine the estimates proved in the previous sections with standard energy estimates in the original $(t, x)$ variables, iterate, and harvest consequences, to complete the proofs of our main results. We mostly give a unified treatment of the cases $k=1$ and $k=2$. To distinguish between the relevant energy densities in the $(t, x)$ and the $y$ variables, in this section we will often use the notation $e_{\varepsilon}(u ; \eta)$ and $e_{\varepsilon}(v ; G)$; see (1-28) and the following discussion.

The next theorem assembles most of our main estimates, and will easily imply Theorems 1 and 2; it can be seen as the main result of this paper. In it, and throughout this section, when we write $C\left(\Gamma, T_{0}\right)$, it will denote a constant that may depend upon various choices made in the construction (2-12) of the map $\psi$ that we use to change variables; these choices, however, are constrained only by $\Gamma$ and $T_{0}$.

Theorem 22. Let $k=1$ or $2, n \geq 1$, and $N=n+k$.

Let $\Gamma \subset(-T, T) \times \mathbb{R}^{N}$ be a smooth timelike Minkowski minimal surface of codimension $k$, satisfying our standing assumptions (2-5)-(2-7). Let $u:(-T, T) \times \mathbb{R}^{N} \rightarrow \mathbb{R}^{k}$ solve (1-1) with initial data satisfying assumptions (2-31) and (2-34)-(2-36), for some $\zeta_{0}$ verifying (2-30).

Given $T_{0}<T$, fix $T_{1} \in\left(T_{0}, T\right)$ and $\rho_{0}>0$ so small that (2-13), (2-14), and the conclusions of Proposition 4 hold on $\left(-T_{1}, T_{1}\right) \times \mathbb{T}^{n} \times B_{v}\left(\rho_{0}\right)$.

There exists a constant $C\left(\Gamma, T_{0}\right)$ such that

$$
\delta_{\varepsilon} \int_{\left(\left(-T_{0}, T_{0}\right) \times \mathbb{R}^{N}\right) \backslash \mathcal{N}} e_{\varepsilon}(u ; \eta) d x d t \leq C \zeta_{0},
$$

for $\mathcal{N}=\operatorname{image}(\psi) \cap\left(\left(-T_{0}, T_{0}\right) \times \mathbb{R}^{N}\right)$, and such that $v=u \circ \psi$ satisfies

$$
\begin{aligned}
& \delta_{\varepsilon} \int_{\left(-T_{1}, T_{1}\right) \times \mathbb{T}^{n} \times B_{v}\left(\rho_{0}\right)}\left|D_{\tau} v\right|^{2}+\left|y^{\nu}\right|^{2}\left(\left|\nabla_{\nu} v\right|^{2}+\frac{1}{\varepsilon^{2}} F(v)\right) d y \leq C \zeta_{0}, \\
& \delta_{\varepsilon} \int_{\left(-T_{1}, T_{1}\right) \times \mathbb{T}^{n} \times B_{v}\left(\rho_{0}\right)}\left(1+\kappa_{2}\left|y^{\nu}\right|^{2}\right) e_{\varepsilon}(v ; G) d y-\mathscr{H}^{1+n}\left(\left(-T_{1}, T_{1}\right) \times \mathbb{T}^{n}\right) \leq C \zeta_{0},
\end{aligned}
$$

$\left(\right.$ for $\kappa_{2}$ as in $(2-23)$, with $\kappa_{2}=1$ when $\left.k=1\right)$, and

$$
\int_{\left(-T_{1}, T_{1}\right) \times \mathbb{T}^{n}} \mathscr{D}_{v}\left(v\left(y^{\tau}\right) ; \rho_{1} / 2\right) d y^{\tau} \leq C \zeta_{0},
$$


where $\mathscr{D}_{v}$ was defined in (3-3) for $k=1$, and in (5-2) for $k=2$, while $\rho_{1}$ was found in Lemma 14. Finally,

$$
\left\|\delta_{\varepsilon} \mathscr{T}_{\varepsilon}(u)-\mathscr{T}(\Gamma)\right\|_{W^{-1,1}\left(\left(-T_{0}, T_{0}\right) \times \mathbb{R}^{N}\right)} \leq C \sqrt{\zeta_{0}}
$$

The following lemma will be used repeatedly.

Lemma 23. There exists a constant $C>0$, depending only on $\Gamma, T_{1}, \rho_{0}$, such that

$$
C^{-1} e_{\varepsilon}(u ; \eta)(\psi(y)) \leq e_{\varepsilon}(v ; G)(y) \leq C e_{\varepsilon}(u ; \eta)(\psi(y))
$$

and

$$
C^{-1} \leq|\operatorname{det} D \psi(y)|=\sqrt{-g(y)} \leq C
$$

for all $y \in\left(-T_{1}, T_{1}\right) \times \mathbb{T}^{n} \times B_{v}\left(\rho_{0}\right)$.

Proof. This is clear from the construction of the diffeomorphism $\psi$, see in particular (2-20).

Next, we show that Theorems 1 and 2 follow directly from Theorem 22 and the above Lemma. The rest of this section will then be devoted to the proof of Theorem 22 .

Proofs of Theorems 1 and 2. First we consider the scalar case, i.e., that of Theorem 1. We define $\mathcal{N}$ as in (2-22). Then, as noted in Corollary 7, the function $d$ defined by (2-29) satisfies the eikonal equation $(1-12)$ in $\mathcal{N}$, as required.

Let $u$ solve (1-1) with the initial data given by Lemma 9, in the case $k=1$, so that it satisfies the assumptions of Theorem 22 with $\zeta_{0}=C \varepsilon^{2}$ and, in addition,

$$
\int_{\mathbb{T}^{n} \times B_{v}\left(\rho_{0}\right)}\left(v_{0}-q\left(\frac{y^{N}}{\varepsilon}\right)\right)^{2} d y^{\prime} \leq C \varepsilon
$$

for $v_{0}$ as defined in (2-33).

Then, conclusion (1-15) of Theorem 1 is exactly (6-5).

To prove (1-14), we recall that $\mathcal{N} \subset \psi\left(\left(-T_{1}, T_{1}\right) \times \mathbb{T}^{n} \times B_{v}\left(\rho_{0}\right)\right)$ and use (2-29) and Lemma 23 to estimate

$$
\begin{aligned}
\delta_{\varepsilon} \int_{\mathcal{N}} d^{2} e_{\varepsilon}(u ; \eta) d x d t & \leq C \delta_{\varepsilon} \int_{\left(-T_{1}, T_{1}\right) \times \mathbb{T}^{n} \times B_{v}\left(\rho_{0}\right)}\left(y^{v}\right)^{2} e_{\varepsilon}(v ; G) d x d t \\
& \leq C \zeta_{0}-\left(\delta_{\varepsilon} \int_{\left(-T_{1}, T_{1}\right) \times \mathbb{T}^{n} \times B_{v}\left(\rho_{0}\right)} e_{\varepsilon}(v ; G) d y-\mathscr{H}^{1+n}\left(\left(-T_{1}, T_{1}\right) \times \mathbb{T}^{n}\right)\right),
\end{aligned}
$$

where the latter inequality is due to (6-3). Next, by using Lemma 11 and arguing exactly as in the proof of (3-17), we see that

$$
2 T_{1}-\delta_{\varepsilon} \int_{\left(-T_{1}, T_{1}\right) \times \mathbb{T}^{n} \times B_{v}\left(\rho_{0}\right)} e_{\varepsilon}(v ; G) d y \leq C \int_{\left(-T_{1}, T_{1}\right) \times \mathbb{T}^{n}} \mathscr{D}_{\nu}\left(v\left(y^{\tau}\right)\right) d y^{\tau}+C e^{-c / \varepsilon} .
$$

The above inequalities and (6-4) imply that $\delta_{\varepsilon} \int_{\mathcal{N}} d^{2} e_{\varepsilon}(u ; \eta) d x d t \leq C \varepsilon^{2}$. By combining this with (6-1), we obtain (1-14).

Finally, to prove (1-13), note that for every $y^{\prime} \in \mathbb{T}^{n} \times B_{v}\left(\rho_{1}\right)$ and $y^{0} \in\left(-T_{1}, T_{1}\right)$,

$$
\left|v\left(y^{0}, y^{\prime}\right)-v_{0}\left(y^{\prime}\right)\right|=\left|v\left(y^{0}, y^{\prime}\right)-v\left(b\left(y^{\prime}\right), y^{\prime}\right)\right| \leq\left|y^{0}-b\left(y^{\prime}\right)\right|^{1 / 2}\left(\int_{-T_{1}}^{T_{1}}\left|\partial_{y^{0}} v\left(s, y^{\prime}\right)\right|^{2} d s\right)^{1 / 2} .
$$


Since $\left|\partial_{y^{0}} v\right| \leq\left|D_{\tau} v\right|$, we find by integrating that

$$
\int_{\left(-T_{1}, T_{1}\right) \times \mathbb{T}^{n} \times B_{v}\left(\rho_{0}\right)}\left|v\left(y^{0}, y^{\prime}\right)-v_{0}\left(y^{\prime}\right)\right|^{2} d y \leq C \int_{\left(-T_{1}, T_{1}\right) \times \mathbb{T}^{n} \times B_{v}\left(\rho_{0}\right)}\left|D_{\tau} v\right|^{2} d y \leq C \varepsilon,
$$

using (6-2) (for the last inequality) and the fact that $\zeta_{0} \leq C \varepsilon^{2}$. Then, (6-6) implies that

$$
\int_{\left(-T_{1}, T_{1}\right) \times \mathbb{T}^{n} \times B_{v}\left(\rho_{0}\right)}\left|v(y)-q\left(y^{N} / \varepsilon\right)\right|^{2} d y \leq C \varepsilon .
$$

By changing variables, using Lemma 23, and recalling (2-29), we obtain (1-13).

The proof of Theorem 2 is much the same, except that we make no claim about $\int\left|v\left(y^{0}, y^{\prime}\right)-v_{0}\left(y^{\prime}\right)\right|^{2}$, as the estimate $\int\left|\partial_{y^{0}} v\right|^{2} d y \leq C$ is too weak to provide good control over this quantity.

Otherwise, we follow the above proof; that is, we let $u$ solve (1-1) with the initial data given by Lemma 9, in the case $k=2$, so that it satisfies the assumptions of Theorem 22 with $\zeta_{0}=C|\ln \varepsilon|^{-1}$. Then, conclusion (1-21) of Theorem 2 is exactly (6-5). To prove (1-20), it suffices, in view of (6-1), to prove that

$$
\int_{\mathcal{N}} \operatorname{dist}(\cdot, \Gamma)^{2} e_{\varepsilon}(u ; \eta) d x d t \leq C .
$$

Using Lemma 23 to change variables, and noting that $\operatorname{dist}(\psi(y), \Gamma)^{2} \leq C\left|y^{\nu}\right|^{2}$ (since the left-hand side is a smooth function of $y$ that vanishes when $y^{v}=0$ ), it suffices to prove that

$$
\int_{\left(-T_{1}, T_{1}\right) \times \mathbb{T}^{n} \times B_{v}\left(\rho_{0}\right)}\left|y^{v}\right|^{2} e_{\varepsilon}(v ; G) d y \leq C .
$$

This follows exactly the proof of (1-14) in the case $k=1$ above. The estimate corresponding to (6-7) has exactly the same form, except that the last term on the right-hand side is now $C|\ln \varepsilon|^{-1}$; this is proved by arguing as before, but using Proposition 17 in place of Lemma 11.

The proof of Theorem 22 will use some standard energy estimates that we now recall.

Lemma 24. Let $u: \mathbb{R}^{1+N} \rightarrow \mathbb{R}^{k}$ be a smooth, finite-energy solution of the semilinear wave equation (1-1). For any $a<b$ and bounded Lipschitz function $\chi: \mathbb{R}^{1+N} \rightarrow \mathbb{R}$,

$$
\left|\int_{\{b\} \times \mathbb{R}^{N}} e_{\varepsilon}(u ; \eta) \chi d x-\int_{\{a\} \times \mathbb{R}^{N}} e_{\varepsilon}(u ; \eta) \chi d x\right| \leq \int_{(a, b) \times \mathbb{R}^{N}} e_{\varepsilon}(u ; \eta)|D \chi| d x d t .
$$

Also, for any pair $a, b$ of real numbers and open $A \subset \mathbb{R}^{N}$,

$$
\int_{\{b\} \times A|b-a|} e_{\varepsilon}(u ; \eta) d x \leq \int_{\{a\} \times A} e_{\varepsilon}(u ; \eta) d x,
$$

where $A_{s}:=\{x \in A: \operatorname{dist}(x, \partial A)>s\}$.

Proof. Both conclusions are standard, and follow from the identity $\partial_{t} e_{\varepsilon}(u ; \eta)=\nabla \cdot\left(u_{t} \nabla u\right)$ satisfied by solutions of (1-1), integration by parts, and the elementary inequality $\left|u_{t} \nabla u\right| \leq e_{\varepsilon}(u ; \eta)$. For the second inequality, assuming for concreteness that $a<b$, it is easy to see that the set $\left\{(t, x): a<t<b, x \in A_{t-a}\right\}$ is a set of finite perimeter, so that the divergence theorem holds and there is no problem in justifying the standard argument. 
Lemma 25. Let $v:\left(-T_{1}, T_{1}\right) \times \mathbb{T}^{n} \times B_{v}\left(\rho_{0}\right) \rightarrow \mathbb{R}^{k}$ be a smooth solution of (2-26). For any $-T_{1} \leq a<$ $b \leq T_{1}$ and $\chi \in W_{0}^{1, \infty}\left(\left(-T_{1}, T_{1}\right) \times \mathbb{T}^{n} \times B_{v}\left(\rho_{0}\right)\right)$,

$$
\left|\int_{\{b\} \times \mathbb{R}^{N}} e_{\varepsilon}(v ; G) \chi d y^{\prime}-\int_{\{a\} \times \mathbb{R}^{N}} e_{\varepsilon}(v ; G) \chi d y^{\prime}\right| \leq C \int_{(a, b) \times \mathbb{R}^{N}} e_{\varepsilon}(v ; G)(|\chi|+|D \chi|) d y .
$$

Proof. Lemma 6 implies that $\partial_{y^{0}} e_{\varepsilon}(v ; G) \leq C e_{\varepsilon}(v ; G)+\nabla \cdot \varphi$, and the positivity (2-16) of the matrix $\left(a^{\alpha \beta}\right)$ together with the definition (2-28) of $\varphi$ imply that $|\varphi| \leq C e_{\varepsilon}(v ; G)$. The conclusion follows from these facts, together with integration by parts, exactly as in the previous lemma.

Now, we present:

Proof of Theorem 22. We treat both cases $k=1$ and $k=2$ simultaneously. We may assume, as usual, that $u$ and $v=u \circ \psi$ are smooth.

It is convenient to define $\rho:\left(-T_{0}, T_{0}\right) \times \mathbb{R}^{N} \rightarrow[0,+\infty]$ by

$$
\rho(t, x)= \begin{cases}\left|y^{v}\right| & \text { if }(t, x)=\psi(y) \\ +\infty & \text { if }(t, x) \notin \mathcal{N}=\operatorname{image}(\psi) .\end{cases}
$$

Note that, when $k=1, \rho(t, x)=|d(t, x)|$ for $(t, x) \in \mathcal{N}$.

Step 1: Given $u:(-T, T) \times \mathbb{R}^{N} \rightarrow \mathbb{R}^{k}$ solving (1-1), $k=1$ or $k=2$, we will say that $u$ is controlled on a set $W \subset\left(-T_{0}, T_{0}\right) \times \mathbb{R}^{N}$ if there exists a constant $C$, depending on $W, \Gamma$, and $\psi$, such that ,for any function $u$ satisfying the hypotheses of Theorem 22,

$$
\int_{W} e_{\varepsilon}(u ; \eta) \leq C \zeta_{0}
$$

(We will only say this about sets that are bounded away from $\Gamma$.) If $W$ is an open set, the integral is understood as $\int \cdots d x d t$ and, if $W$ is a subset of some $\{t\} \times \mathbb{R}^{N}$, it is understood as $\int \cdots d x$.

Similarly, for a set $W \subset\left(-T_{1}, T_{1}\right) \times \mathbb{T}^{n} \times B_{v}\left(\rho_{0}\right)$, we say that $v=u \circ \psi$ is controlled on $W$ if there exists a constant $C=C\left(W, \Gamma, T_{0}\right)$ such that

$$
\delta_{\varepsilon} \int_{W}\left(\left|D_{\tau} v\right|^{2}+\left|y^{\nu}\right|^{2}\left(\left|\nabla_{\nu} v\right|^{2}+\frac{1}{\varepsilon^{2}} F(v)\right)\right) \leq C \zeta_{0},
$$

Again, $W$ may be either an open set or a subset of $\{s\} \times \mathbb{T}^{n} \times B_{v}\left(\rho_{0}\right)$ for some $s$, with the integral understood accordingly.

We make some easy remarks. First, if $v$ is controlled on a set $W$, then, since

$$
e_{\varepsilon}(v ; G) \leq C(\hat{\rho})\left(\left|D_{\tau} v\right|^{2}+\left|y^{v}\right|^{2}\left(\left|\nabla_{\nu} v\right|^{2}+\frac{1}{\varepsilon^{2}} F(v)\right)\right) \quad \text { whenever }\left|y^{v}\right| \geq \hat{\rho},
$$

it follows that, for any $\hat{\rho} \in\left(0, \rho_{0}\right)$,

$$
\int_{\left\{y=\left(y^{\tau}, y^{v}\right) \in W:\left|y^{v}\right| \geq \hat{\rho}\right\}} e_{\varepsilon}(v ; G) \leq C \zeta_{0} .
$$

As a result, Lemma 23 implies that, if $A \subset \operatorname{Image}(\psi)$ is bounded away from $\Gamma$, then $u$ is controlled on $A$ if and only if $v$ is controlled on $\psi^{-1}(A)$, 
Finally, we remark that, for any $\hat{\rho}>0$, the assumptions (2-31), (2-34), (2-35), and a change of variables imply that

$$
u \text { is controlled on }\left\{(0, x) \in \mathbb{R}^{1+N}: \rho(0, x) \geq \hat{\rho}\right\},
$$

with the implicit constants depending on $\hat{\rho}$ and $\psi$ or, more precisely, on the behavior of $\psi$ on $\{y \in$ $\left.\left(-T_{1}, T_{1}\right) \times \mathbb{T}^{n} \times B_{v}\left(\rho_{0}\right): \psi^{0}(y)=0\right\}$.

Step 2: We next claim that for $0<s_{+}^{\prime}<s_{+} \leq T_{1}$ and $\rho^{\prime}<\rho_{1} / 2$,

if $v$ is controlled on $\left\{y \in\left(-T_{1}, s_{+}\right) \times \mathbb{T}^{n} \times B_{v}\left(\rho_{1} / 2\right): \psi^{0}(y)>0\right\}$,

then, for every $t \in\left[0, s_{+}^{\prime}\right], u$ is controlled on $\left\{(t, x): \rho(t, x)>\rho^{\prime}\right\}$,

and this control is uniform for $t \in\left[0, s_{+}^{\prime}\right]$. To prove this, we fix $\hat{\rho}>0$ so small that $\hat{\rho} \leq \rho^{\prime}$ and

$$
\begin{aligned}
\left\{(t, x): 0<t<s_{+}^{\prime}, \rho(t, x)<\hat{\rho}\right\}=\left\{\psi(y): y \in\left(-T_{1}, T_{1}\right) \times \mathbb{T}^{n} \times B_{v}(\hat{\rho}), 0<\psi^{0}(y)<s_{+}^{\prime}\right\} \\
\\
\subset\left\{\psi(y): y \in\left(-T_{1}, s_{+}\right) \times \mathbb{T}^{n} \times B_{v}(\hat{\rho}): \psi^{0}(y)>0\right\} .
\end{aligned}
$$

The point is that, if $\left|y^{\nu}\right|$ is small enough and $\psi^{0}(y)<s_{+}^{\prime}$, then $y^{0}<s_{+}$. Such a number $\hat{\rho}$ exists, because $\left|\psi^{0}\left(y^{0}, y^{\tau \prime}, y^{\nu}\right)-y^{0}\right| \leq C\left|y^{\nu}\right|$; this is an easy consequence of the definition of $\psi$.

Then, it follows from the control of $v$ and Step 1 that

$$
u \text { is controlled on }\left\{(t, x): 0<t<s_{+}^{\prime}, \hat{\rho} / 2<\rho(t, x)<\hat{\rho}\right\},
$$

since this set is bounded away from $\Gamma$ and is contained, by the choice of $\hat{\rho}$, in the image via $\psi$ of a set on which we have assumed that $v$ is controlled.

Now, we fix a function $\chi \in C^{\infty}\left(\left[0, s_{+}^{\prime}\right] \times \mathbb{R}^{N}\right)$ such that $\chi=1$ wherever $\rho(t, x)>\hat{\rho}$, and $\chi=0$ where $\rho(t, x) \leq \hat{\rho} / 2$. Then, we apply (6-8) with this choice of $\chi$ and with $a=0$ and $b \in\left(0, s_{+}^{\prime}\right)$ and, using (6-10) and (6-12), we find that $u$ is controlled on $\{(b, x): \rho(b, x)>\hat{\rho}\}$, with implicit constants that are uniform for $b \in\left(0, s_{+}^{\prime}\right]$. Thus, we have proved (6-11).

Step 3: We next claim that, for $0<s_{+} \leq T_{1}$ as above,

$$
\begin{aligned}
& \text { if } v \text { is controlled on }\left\{y \in\left(-T_{1}, s_{+}\right) \times \mathbb{T}^{n} \times B_{v}\left(\rho_{1} / 2\right): \psi^{0}(y)>0\right\} \text {, } \\
& \text { then } v \text { is controlled on }\left\{s_{+}\right\} \times \mathbb{T}^{n} \times\left(B_{v}\left(\rho_{1}\right) \backslash B_{v}\left(\rho_{1} / 2\right)\right) .
\end{aligned}
$$

We first apply (6-11), with parameters $s_{+}^{\prime}<s_{+}$and $\rho^{\prime}<\frac{1}{2} \rho_{1}$ to be fixed later. We then apply (6-9) with $a=s_{+}^{\prime}$ and $|b| \leq T$, to conclude that $u$ is controlled on

$$
S\left(s_{+}^{\prime}, \rho^{\prime}\right):=\left\{(t, x):|t| \leq T, \operatorname{dist}\left(x, A\left(s_{+}^{\prime}, \rho^{\prime}\right)\right)>\left|t-s_{+}^{\prime}\right|\right\}
$$

where

$$
A\left(s_{+}^{\prime}, \rho^{\prime}\right):=\left\{x: \rho\left(s_{+}^{\prime}, x\right)>\rho^{\prime}\right\} .
$$

Then, Step 1 implies that $v$ is controlled on $\psi^{-1}\left(S\left(s_{+}^{\prime}, \rho^{\prime}\right)\right)$.

We will show that we can fix $s_{+}^{\prime}<s_{+}$and $\rho^{\prime}>0$ such that

$$
\psi\left(\left\{s_{+}\right\} \times \mathbb{T}^{n} \times\left(B_{v}\left(\rho_{0}\right) \backslash B_{v}\left(\rho_{1} / 4\right)\right)\right) \Subset S\left(s_{+}^{\prime}, \rho^{\prime}\right),
$$

by which we mean that some open neighborhood of $\psi(\cdots)$ is contained in $S\left(s_{+}^{\prime}, \rho^{\prime}\right)$. For now, we assume that we have selected $s_{+}^{\prime}$ and $\rho^{\prime}$ so that (6-14) holds, and we complete the proof of (6-13). 
Indeed, if (6-14) holds, then clearly

$$
\left\{s_{+}\right\} \times \mathbb{T}^{n} \times\left(B_{v}\left(\rho_{0}\right) \backslash B_{v}\left(\rho_{1} / 4\right)\right) \Subset \psi^{-1}\left(S\left(s_{+}^{\prime}, \rho^{\prime}\right)\right) .
$$

and, so, there exists some $a<s_{+}$such that $\left(a, s_{+}\right) \times \mathbb{T}^{n} \times\left(B_{v}\left(\rho_{0}\right) \backslash B_{v}\left(\rho_{1} / 4\right)\right) \Subset \psi^{-1}\left(S\left(s_{+}^{\prime}, \rho^{\prime}\right)\right)$. Now, we can find some smooth nonnegative function $\chi$ such that

$$
\chi=1 \text { on }\left\{s_{+}\right\} \times \mathbb{T}^{n} \times\left(\bar{B}_{v}\left(\rho_{1}\right) \backslash B_{v}\left(\rho_{1} / 2\right)\right) \quad \text { and } \operatorname{spt}(\chi) \subset\left(a, T_{1}\right) \times \mathbb{T}^{n} \times\left(B_{v}\left(\rho_{0}\right) \backslash B_{v}\left(\rho_{1} / 4\right)\right) .
$$

Since $v$ is controlled in $\psi^{-1}\left(S\left(s_{+}^{\prime}, \rho^{\prime}\right)\right)$, (6-13) follows from applying Lemma 25 with this choice of $\chi$ and $a$, and with $b=s_{+}$.

Step 4: We next verify (6-14). Since the sets $S(s, \rho)$ depend continuously on $s$ and $\rho$ in an obvious way, (6-14) will follow (for suitable $s_{+}^{\prime}<s_{+}$and $\rho^{\prime}>0$ ) if we can show that

$$
\psi\left(\left\{s_{+}\right\} \times \mathbb{T}^{n} \times\left(B_{v}\left(\rho_{0}\right) \backslash B_{v}\left(\rho_{1} / 4\right)\right)\right) \Subset S\left(s_{+}, 0\right) .
$$

We will deduce this as a consequence of the following fact: If $\Sigma$ is a connected spacelike hypersurface in $(1+N)$-dimensional Minkowski space, and we define the solid light cone with vertex $(t, x)$ to be

$$
L C(t, x):=\left\{\left(t^{\prime}, x^{\prime}\right):\left|x-x^{\prime}\right| \geq\left|t-t^{\prime}\right|\right\},
$$

then $L C(t, x) \cap \Sigma=\{(t, x)\}$ for every $(t, x) \in \Sigma$.

To reduce (6-15) to this geometric fact, we define

$$
\Sigma:=\psi\left(\left\{s_{+}\right\} \times \mathbb{T}^{n} \times B_{v}\left(\rho_{0}\right)\right) .
$$

Clearly, $\Sigma$ is a connected hypersurface. We claim that it is also spacelike. To see this, recall (see (2-20)) that $\left(g_{i j}\right)_{i, j=1}^{N}$ is positive definite; this implies that $\psi^{-1}(\Sigma)=\left\{s_{+}\right\} \times \mathbb{T}^{n} \times B_{v}\left(\rho_{0}\right)$ is spacelike with respect to the $\left(g_{\alpha \beta}\right)$ metric. The claim then follows, since $\psi$ is an isometry between $\left(-T_{1}, T_{1}\right) \times \mathbb{T}^{n} \times B_{v}\left(\rho_{0}\right)$ with the $\left(g_{\alpha \beta}\right)$ metric and image $(\psi) \subset \mathbb{R}^{1+N}$ with the Minkowski metric in standard form $d s^{2}=-d t^{2}+$ $\left(d x^{1}\right)^{2}+\ldots+\left(d x^{N}\right)^{2}$.

Next, note that the definition of $\psi$ and the choice (2-13) of $\rho_{0}$ imply that

$$
\psi\left(\left\{s_{+}\right\} \times \mathbb{T}^{n} \times\left(B_{v}\left(\rho_{0}\right) \backslash B_{v}\left(\rho_{1} / 4\right)\right)\right) \Subset(-T, T) \times \mathbb{R}^{N} .
$$

Thus, in order to prove (6-15), it suffices to show that the closure of $\psi\left(\left\{s_{+}\right\} \times \mathbb{T}^{n} \times\left(B_{v}\left(\rho_{0}\right) \backslash B_{v}\left(\rho_{1} / 4\right)\right)\right)$ does not intersect $\left((-T, T) \times \mathbb{R}^{N}\right) \backslash S\left(s_{+}, 0\right)$. However, by inspection of the definition of $S(s, \rho)$, one sees that

$$
\left((-T, T) \times \mathbb{R}^{N}\right) \backslash S\left(s_{+}, 0\right) \subset \underset{\left\{\left(x \in \mathbb{R}^{N}: \rho\left(s_{+}, x\right)=0\right\}\right.}{\bigcup} L C\left(s_{+}, x\right)=\bigcup_{\left\{x \in \mathbb{R}^{N}:\left(s_{+}, x\right) \in \Gamma\right\}} L C\left(s_{+}, x\right) .
$$

In addition, since $\Gamma \cap\left(\left\{s_{+}\right\} \times \mathbb{R}^{N}\right)=\psi\left(\left\{s_{+}\right\} \times \mathbb{T}^{n} \times\{0\}\right)$, it is clear that

$$
\Sigma \supset \Gamma \cap\left(\left\{s_{+}\right\} \times \mathbb{R}^{N}\right) .
$$

Then, the geometric fact mentioned above implies that

$$
\Sigma \cap\left(\left((-T, T) \times \mathbb{R}^{N}\right) \backslash S\left(s_{+}, 0\right)\right) \subset \underset{\left\{x:\left(s_{+}, x\right) \in \Gamma\right\}}{\bigcup} \Sigma \cap L C\left(s_{+}, x\right)=\Gamma \cap\left(\left\{s_{+}\right\} \times \mathbb{R}^{N}\right)=\psi\left(\left\{s_{+}\right\} \times \mathbb{T}^{n} \times\{0\}\right) .
$$


Since $\psi$ is injective, this implies that $\psi\left(\left\{s_{+}\right\} \times \mathbb{T}^{n} \times\left(B_{v}\left(\rho_{0}\right) \backslash\{0\}\right)\right)$ does not intersect $\left((-T, T) \times \mathbb{R}^{N}\right) \backslash$ $S\left(s_{+}, 0\right)$, completing the proof of (6-15).

$\underline{\text { Step 5: }}$ We introduce more terminology. For a set $W \subset\left(-T_{1}, T_{1}\right) \times \mathbb{T}^{n} \times B_{v}\left(\rho_{0}\right)$, if there exists $W_{\tau} \subset$ $\left(-T_{1}, T_{1}\right) \times \mathbb{T}^{n}$ such that

$$
W_{\tau} \times B_{v}\left(\rho_{1} / 2\right) \subset W \subset W_{\tau} \times B_{v}\left(\rho_{0}\right),
$$

then we say that $v$ is completely controlled on $W$ if $v$ is controlled on $W$ and, in addition, there exists a constant $C(W, \psi)$ such that

$$
\delta_{\varepsilon} \int_{W}\left(\left(1+\kappa_{2}\left|y^{\nu}\right|^{2}\right) e_{\varepsilon}(v ; G)\right)-\mathscr{H}^{\operatorname{dim} W_{\tau}}\left(W_{\tau}\right) \leq C \zeta_{0} \quad \text { and } \quad \int_{W_{\tau}} \mathscr{D}_{\nu}\left(v\left(y^{\tau}\right)\right) \leq C \zeta_{0} .
$$

And, as above, we allow $W$ to be either an open set or a subset of some $\left\{y^{0}=\right.$ constant $\}$ slice, with the integral understood accordingly, and with $\operatorname{dim} W_{\tau}=1+n$ in the first case and $\operatorname{dim} W_{\tau}=n$ in the second.

Now, we establish estimates (6-1)-(6-4). First, by assumption, $v=u \circ \psi$ satisfies the hypotheses of Proposition 13 (if $k=1$ ) and Proposition 16 (if $k=2$ ), and these imply that

$$
\begin{aligned}
& v \text { is controlled on }\left\{y \in\left(-T_{1}, s_{1}\right) \times \mathbb{T}^{n} \times B_{v}\left(\rho_{1}\right): \psi^{0}(y)>0\right\}, \quad \text { and } \\
& v \text { is completely controlled on }\left\{s_{1}\right\} \times \mathbb{T}^{n} \times B_{v}\left(\rho_{1}\right) .
\end{aligned}
$$

In particular, (6-17) implies that $v$ satisfies the hypotheses of Proposition $10(k=1)$ or Proposition 15 $(k=2)$, with $\zeta_{0}$ replaced by $C \zeta_{0}$. These propositions assert that

$$
v \text { is completely controlled on }\{s\} \times \mathbb{T}^{n} \times B_{v}\left(\rho_{1} / 2\right) \text { for } s_{1} \leq s \leq s_{2},
$$

where $s_{2}:=\min \left\{T_{1}, s_{1}+\left(\rho_{1} / 2 c_{*}\right)\right\}$. Then, (6-16) and (6-18) imply that $v$ is controlled on

$$
\left\{y \in\left(-T_{1}, s_{2}\right) \times \mathbb{T}^{n} \times B_{v}\left(\rho_{1} / 2\right): \psi^{0}(y)>0\right\} .
$$

Next, we invoke (6-13) to find that $v$ is controlled on $\left\{s_{2}\right\} \times \mathbb{T}^{n} \times\left(B_{v}\left(\rho_{1}\right) \backslash B_{v}\left(\rho_{1} / 2\right)\right)$. Hence, appealing again to (6-18), we see that $v$ is completely controlled on $\left\{s_{2}\right\} \times \mathbb{T}^{n} \times B_{v}\left(\rho_{1}\right)$.

Thus, we can apply Proposition 10 or 15 , with $s_{1}$ replaced by $s_{2}$ and $\zeta_{0}$ multiplied by a suitable constant, but with the same fixed valued of $\rho_{1}$ already used. We can repeat this argument as necessary to find, after a finite number of iterations, that $v$ is completely controlled on $\{s\} \times \mathbb{T}^{n} \times B_{v}\left(\rho_{1} / 2\right)$ for $s_{1} \leq s \leq T_{1}$. Since all our energy estimates are clearly valid backwards in the timelike variables, we can also iterate Proposition 10 or 15 backwards, starting from $s_{1}$ and arguing as above, to conclude that

$$
v \text { is completely controlled on }\{s\} \times \mathbb{T}^{n} \times B_{v}\left(\rho_{1} / 2\right) \text { for }-T_{1} \leq s \leq T_{1} \text {. }
$$

Since $T_{1}>T_{0}$, we deduce, by applying (6-11) in both directions in the $t$ variable, that

$$
u \text { is controlled on }\left\{(t, x) \in\left(-T_{0}, T_{0}\right) \times \mathbb{R}^{N}: \rho(t, x) \geq \rho_{1} / 4\right\} .
$$

Using these and Lemma 24, we can deduce (arguing as in the proof of (6-11) and (6-13)) that in fact $v$ is completely controlled on $\left(-T_{1}, T_{1}\right) \times \mathbb{T}^{n} \times B_{v}\left(\rho_{0}\right)$. These estimates imply (6-1)-(6-4).

Step 6: It remains to prove (6-5). The point is that it essentially suffices to prove the same estimate in the $y$ variables, in which (6-2)-(6-4) imply a great deal of information about the way in which energy 
concentrates around $\Gamma$, which in these variables is $\left(-T_{1}, T_{1}\right) \times \mathbb{T}^{n} \times\{0\}$. We will extract this information using Lemma 11 for the case $k=1$, and an estimate of [Kurzke and Spirn 2009] for $k=2$.

If $m=\left(m_{\alpha}^{\beta}\right)$ and $\mathscr{T}=\left(\mathscr{T}_{\beta}^{\alpha}\right)$, let us write

$$
\langle m, \mathscr{T}\rangle:=\int m_{\alpha}^{\beta} d \mathscr{T}_{\beta}^{\alpha} .
$$

Then, we must estimate $\left\langle m, \delta_{\varepsilon} \mathscr{T}_{\varepsilon}(u)-\mathscr{T}(\Gamma)\right\rangle$ for $\left(m_{\alpha}^{\beta}\right) \in W^{1, \infty}\left(\left(-T_{0}, T_{0}\right) \times \mathbb{R}^{N}\right)$, with compact support and with $\|m\|_{W^{1, \infty}} \leq 1$. To do this, let $\chi$ be a smooth function with support in image $(\psi)$ and such that $\chi=1$ on $\left\{(t, x):|t|<T_{0}, \rho(t, x)<\rho_{0} / 2\right\}$. Then,

$$
\left\langle m, \mathscr{T}_{\varepsilon}(u)-\mathscr{T}(\Gamma)\right\rangle=\left\langle(1-\chi) m, \mathscr{T}_{\varepsilon}(u)\right\rangle+\left\langle\chi m, \mathscr{T}_{\varepsilon}(u)-\mathscr{T}(\Gamma)\right\rangle .
$$

It is clear from the definition $(2-8)$ of $\mathscr{T}_{\varepsilon}$ that $\left|\mathscr{T}_{\varepsilon, \beta}^{\alpha}(u)\right| \leq C e_{\varepsilon}(u ; \eta)$, so that

$$
\begin{aligned}
\left.\|(1-\chi) m, \mathscr{T}_{\varepsilon}(u)\right\rangle \mid & \leq \sum_{\alpha, \beta}\left\|m_{\alpha}^{\beta}\right\|_{\infty} \int_{\left\{(t, x) \in\left(-T_{0}, T_{0}\right) \times \mathbb{R}^{N}: \rho(t, x) \geq \rho_{0} / 2\right\}} e_{\varepsilon}(u ; \eta) d t d x \\
& \leq C \zeta_{0},
\end{aligned}
$$

using (6-1) and (6-2) together with Lemma 23.

Step 7: Let us write $\bar{m}:=\chi m$. Note that $\bar{m}$ is supported in image $(\psi)$, and $\|\bar{m}\|_{W^{1, \infty}} \leq C$. We will write

$$
\check{m}_{\delta}^{\gamma}(y)=\bar{m}_{\alpha}^{\beta} \circ \psi(y) \psi_{y_{\delta}}^{\alpha}(y) \varphi_{x^{\beta}}^{\gamma} \circ \psi(y) \sqrt{-g(y)} \quad \text { and } \quad \varphi:=\psi^{-1} \text { as usual. }
$$

Note that $\|\check{m}\|_{W^{1, \infty}} \leq C$. We claim that

$$
\left\langle\bar{m}, \mathscr{T}_{\varepsilon}(u)\right\rangle=\int_{\left(-T_{1}, T_{1}\right) \times \mathbb{T}^{n} \times B_{v}\left(\rho_{0}\right)} \check{m}_{\delta}^{\gamma}(y) \tilde{\mathscr{T}}_{\varepsilon, \gamma}^{\delta}(v)(y) d y
$$

and

$$
\langle\bar{m}, \mathscr{T}(\Gamma)\rangle=\int_{\left(-T_{1}, T_{1}\right) \times \mathbb{T}^{n}} \check{m}_{\delta}^{\gamma}\left(y^{\tau}, 0\right) \tilde{P}_{\gamma}^{\delta} d y^{\tau},
$$

where $\tilde{\mathscr{T}}_{\varepsilon}(v)$ was defined $^{9}$ in (4-12), $\tilde{P}_{\gamma}^{\delta}=1$ if $\delta=\gamma \in\{0, \ldots, n\}$ and $\tilde{P}_{\gamma}^{\delta}=0$ otherwise. These are arguably obvious from the tensorial nature of the quantities involved. However, for the convenience of the reader, we note that the definitions (2-8), (4-12) and (6-23) imply that

$$
\bar{m}_{\alpha}^{\beta}(t, x) \mathscr{T}_{\varepsilon, \beta}^{\alpha}(u)(t, x)=\check{m}_{\alpha}^{\beta}(y) \tilde{\mathscr{T}}_{\varepsilon, \beta}^{\alpha}(v)(y)(-g(y))^{-1 / 2} \text { for }(t, x)=\psi(y) .
$$

Then, (6-24) follows from a change of variables, noting that $|\operatorname{det} D \psi|=\sqrt{-g}$, so $d t d x=\sqrt{-g(y)} d y$. To rewrite $\langle\bar{m}, \mathcal{T}(\Gamma)\rangle$, note that our proof of Lemma 3 (to which we refer for notation) showed that

$$
\langle\bar{m}, \mathscr{T}(\Gamma)\rangle=\int_{(-T, T) \times \mathbb{T}^{n}}\left(\bar{m}_{\alpha}^{\beta} \circ H\right) H_{y^{a}}^{\alpha} \eta_{\beta \delta} H_{y^{b}}^{\delta} \gamma^{a b} \sqrt{-\gamma} d y^{\tau},
$$

\footnotetext{
${ }^{9}$ As remarked earlier, $\widetilde{\mathscr{T}}_{\varepsilon}(v)$ is just the energy-momentum tensor for $u$, expressed in terms of the $y$ variables.
} 
where $H:(-T, T) \times \mathbb{T}^{n} \rightarrow(-T, T) \times \mathbb{R}^{N}$ is the given map parametrizing $\Gamma$ (see (2-5)), and with $a, b$ summed implicitly from 0 to $n$. Since $\psi\left(y^{\tau}, 0\right)=H\left(y^{\tau}\right)$, we can rewrite the integrand above in terms of $\psi$ and $g$, and this leads to (6-25). For this, it is useful to note that

$$
g_{\alpha \beta}\left(y^{\tau}, 0\right)= \begin{cases}\gamma_{\alpha \beta}\left(y^{\tau}\right) & \text { if } \alpha, \beta \leq n, \\ \delta_{\alpha \beta} & \text { if } \alpha, \beta>n, \\ 0 & \text { otherwise, }\end{cases}
$$

and that

$$
g^{a b} \psi_{y_{b}}^{\delta} \eta_{\delta \beta}=\varphi_{\alpha}^{a} \circ \psi \eta^{\alpha \gamma} \varphi_{\gamma}^{b} \circ \psi \psi_{y_{b}}^{\delta} \eta_{\delta \beta}=\varphi_{x^{\beta}}^{a} \circ \psi
$$

for $a \in\{0, \ldots, n\}$.

$\underline{\text { Step 8: }}$ We now apply our earlier estimates to control various terms in $\left\langle\bar{m}, \mathscr{T}_{\varepsilon}(u)-\mathscr{T}(\Gamma)\right\rangle$ represented, as in (6-24) and (6-25), in terms of the $y$ coordinates. In these calculations, we do not sum over indices $\gamma$ and $\delta$ when they are repeated.

Case 1: $\gamma \neq \delta, \delta \leq n$ : When this holds, we have, using successively (4-12) and (2-19),

$$
\begin{aligned}
\left|\widetilde{T}_{\varepsilon, \gamma}^{\delta}\right| & =\left|g^{\delta \alpha} v_{y^{\alpha}} v_{y^{\gamma}}\right| \\
& \leq C\left(\left|D_{\tau} v\right|^{2}+\left|y^{\nu}\right|^{2}\left|\nabla_{\nu} v\right|^{2}\right) .
\end{aligned}
$$

Thus, in this case,

$$
\delta_{\varepsilon} \int_{\left(-T_{1}, T_{1}\right) \times \mathbb{T}^{n} \times B_{\nu}\left(\rho_{0}\right)} \check{m}_{\delta}^{\gamma} \widetilde{\mathscr{T}}_{\varepsilon, \gamma}^{\delta} d y \leq C\|\check{m}\|_{\infty} \zeta_{0},
$$

where we have used (6-2).

Case 2: $\gamma \neq \delta, \gamma \leq n$ : In this case, we have the weaker estimate

$$
\begin{aligned}
\left|\widetilde{T}_{\varepsilon, \gamma}^{\delta}\right| & =\left|g^{\delta \alpha} v_{y^{\alpha}} v_{y^{\gamma}}\right| \\
& \leq C\left(\left|D_{\tau} v\right|^{2}+\left|D_{\tau} v\right|\left|\nabla_{\nu} v\right|\right),
\end{aligned}
$$

again using (4-12) and (2-19). So, we have

$$
\begin{aligned}
\delta_{\varepsilon} \int_{\left(-T_{1}, T_{1}\right) \times \mathbb{I}^{n} \times B_{v}\left(\rho_{0}\right)}^{\check{m}_{\delta}^{\gamma} \widetilde{\mathfrak{T}}_{\varepsilon, \gamma}^{\delta} d y} & \leq C \delta_{\varepsilon}\|\check{m}\|_{\infty}\left(\left\|D_{\tau} v\right\|_{2}^{2}+\left\|D_{\tau} v\right\|_{2}\left\|\nabla_{v} v\right\|_{2}\right) \\
& \leq C\|\check{m}\|_{\infty}\left(\zeta_{0}+\sqrt{\zeta_{0}} \sqrt{\delta_{\varepsilon}}\left\|\nabla_{\nu} v\right\|_{2}\right) \quad \text { (using (6-2)) } \\
& \leq C\|\check{m}\|_{\infty}\left(\zeta_{0}+\sqrt{\zeta_{0}} \sqrt{\left(\zeta_{0}+2 T_{1}\right)}\right) \quad \text { (using (6-3)). }
\end{aligned}
$$

Case 3: $\gamma=\delta \leq n$ : This is the only case in which $\langle m, \mathscr{T}(\Gamma)\rangle$ makes a nonzero contribution. Indeed, by (2-19), $\left|g^{\delta \alpha} v_{y^{\alpha}} v_{y^{\delta}}\right| \leq C\left(\left|D_{\tau} v\right|^{2}+\left|D_{\tau} v\right|\left|\nabla_{\nu} v\right|\right)$, so that

$$
\begin{aligned}
\widetilde{T}_{\varepsilon, \delta}^{\delta} & =\frac{1}{2} g^{\alpha \beta} v_{y_{\alpha}} v_{y^{\beta}}+\frac{1}{\varepsilon^{2}} F(v)+O\left(\left|D_{\tau} v\right|^{2}+\left|D_{\tau} v\right|\left|\nabla_{v} v\right|\right) \\
& =\frac{1}{2}\left|\nabla_{\nu} v\right|^{2}+\frac{1}{\varepsilon^{2}} F(v)+O\left(\left|D_{\tau} v\right|^{2}+\left|D_{\tau} v\right|\left|\nabla_{\nu} v\right|\right),
\end{aligned}
$$

using (2-19) and (2-20). Thus,

$$
\delta_{\varepsilon} \int_{\left(-T_{1}, T_{1}\right) \times \mathbb{T}^{n} \times B_{v}\left(\rho_{0}\right)} \check{m}_{\delta}^{\delta} \widetilde{\mathfrak{T}}_{\varepsilon, \delta}^{\delta} d y=\delta_{\varepsilon} \int_{\left(-T_{1}, T_{1}\right) \times \mathbb{T}^{n} \times B_{v}\left(\rho_{0}\right)} \check{m}_{\delta}^{\delta} e_{\varepsilon, v}(v) d y+O\left(\|\check{m}\|_{\infty} \sqrt{\zeta_{0}}\right) .
$$


The contribution to $\left\langle\bar{m}, \mathscr{T}_{\varepsilon}(u)-\mathscr{T}(\Gamma)\right\rangle$ from a summand with $\delta=\gamma \leq n$ is, thus,

$$
\begin{gathered}
\int_{\left(-T_{1}, T_{1}\right) \times \mathbb{T}^{n} \times B_{v}\left(\rho_{0}\right)} \check{m}_{\delta}^{\delta} \delta_{\varepsilon} e_{\varepsilon, v}(v) d y-\int_{\left(-T_{1}, T_{1}\right) \times \mathbb{T}^{n}} m_{\delta}^{\delta}\left(y^{\tau}, 0\right) d y^{\tau}+O\left(\|\check{m}\|_{\infty} \sqrt{\zeta_{0}}\right) \\
=\int_{\left(-T_{1}, T_{1}\right) \times \mathbb{\Psi}^{n} \times B_{v}\left(\rho_{0}\right)}\left(\check{m}_{\delta}^{\delta}\left(y^{\tau}, y^{v}\right)-\check{m}_{\delta}^{\delta}\left(y^{\tau}, 0\right)\right) \delta_{\varepsilon} e_{\varepsilon, v}(v) d y \\
\quad-\int_{\left(-T_{1}, T_{1}\right) \times \mathbb{\Psi}^{n}} m_{\delta}^{\delta}\left(y^{\tau}, 0\right)\left(1-\delta_{\varepsilon} \int_{B_{v}(\rho)} e_{\varepsilon, v}(v)\left(y^{\tau}, y^{v}\right) d y^{v}\right) d y^{\tau}+O\left(\|\check{m}\|_{\infty} \sqrt{\zeta_{0}}\right) \\
=: A+B+O\left(\|\check{m}\|_{\infty} \sqrt{\zeta_{0}}\right) .
\end{gathered}
$$

To estimate $A$, note that $\left|\check{m}_{\delta}^{\delta}\left(y^{\tau}, y^{\nu}\right)-\check{m}_{\delta}^{\delta}\left(y^{\tau}, 0\right)\right| \leq\|\check{m}\|_{W^{1, \infty}}\left|y^{v}\right| \leq C\left|y^{v}\right|$, so that

$$
|A| \leq\left(\delta_{\varepsilon} \int\left|y^{v}\right|^{2} e_{\varepsilon, v}(v) d y\right)^{1 / 2}\left(\delta_{\varepsilon} \int e_{\varepsilon, v}(v) d y\right)^{1 / 2} \leq C \sqrt{\zeta_{0}}
$$

after arguing, as in Case 2 above, to estimate $\int e_{\varepsilon, v}(v) \leq C$.

As for the other term, since $\|\check{m}\|_{\infty} \leq C$,

$$
|B| \leq C \int_{\left(-T_{1}, T_{1}\right) \times \mathbb{T}^{n}}\left|\Theta_{1}\left(y^{\tau}\right)\right| d y^{\tau} \quad \text { for } \Theta_{1}\left(y^{\tau}\right):=\delta_{\varepsilon} \int_{B_{v}(\rho)} e_{\varepsilon, v}(v)\left(y^{\tau}, y^{v}\right) d y^{v}-1
$$

We say that $y^{\tau}$ is $\operatorname{good}$ if $\mathscr{D}_{v}\left(v\left(y^{\tau}\right)\right) \leq \kappa_{3}$, where $\kappa_{3}$ is the constant from Lemma 11 and Proposition 17, for $k=1$ and $k=2$, respectively. A point will be called bad if it is not good. In particular, these results show that if $y^{\tau}$ is good, then

$$
\Theta_{1}\left(y^{\tau}\right) \geq\left\{\begin{array}{ll}
-C e^{-c / \varepsilon} & \text { if } k=1 \\
-C|\ln \varepsilon|^{-1} & \text { if } k=2
\end{array}\right\} \geq-C \zeta_{0} \text { in both cases, }
$$

since $\delta_{\varepsilon} \leq \zeta_{0}$. Thus, since clearly $\Theta_{1}\left(y^{\tau}\right) \geq-1$ everywhere, we see that

$$
\left|\Theta_{1}\left(y^{\tau}\right)\right| \leq \begin{cases}\Theta_{1}\left(y^{\tau}\right)+C \zeta_{0} & \text { if } y^{\tau} \text { is good } \\ \Theta_{1}\left(y^{\tau}\right)+2 & \text { if } y^{\tau} \text { is bad }\end{cases}
$$

Thus, we compute

$$
\begin{aligned}
|B| & \leq C \int_{\{\text {good points }\}}\left(\Theta_{1}\left(y^{\tau}\right)+C \zeta_{0}\right) d y^{\tau}+C \int_{\{\text {bad points }\}}\left(\Theta_{1}\left(y^{\tau}\right)+2\right) d y^{\tau} \\
& \leq C \int_{\left(-T_{0}, T_{0}\right) \times \mathbb{T}^{n}} \Theta_{1}\left(y^{\tau}\right) d y^{\tau}+C \zeta_{0}+2 \mathscr{H}^{1+n}\left(\left\{y^{\tau} \in\left(-T_{0}, T_{0}\right) \times \mathbb{T}^{n}: y^{\tau} \text { is bad }\right\}\right) .
\end{aligned}
$$

To conclude the estimate, we note that (6-3) implies that $\int_{\left(-T_{0}, T_{0}\right) \times \mathbb{\mathbb { T }}^{n}} \Theta_{1}\left(y^{\tau}\right) d y^{\tau} \leq C \zeta_{0}$, and (6-4), together with Chebyshev's inequality, implies that

$$
\mathscr{H}^{1+n}\left(\left\{y^{\tau} \in\left(-T_{0}, T_{0}\right) \times \mathbb{T}^{n}: y^{\tau} \text { is bad }\right\}\right) \leq C \int_{\left(-T_{0}, T_{0}\right) \times \mathbb{T}^{n}} \mathscr{D}_{\nu}\left(v\left(y^{\tau}, \cdot\right)\right) d y^{\tau} \leq C \zeta_{0} .
$$

Thus, $|B| \leq C \zeta_{0}$.

Case 4: $\gamma, \delta>n$ : Here, we consider the cases $k=1$ and $k=2$ separately. 
$\underline{k=1}$ : The assumption of Case 4 reduces to $\gamma=\delta=N$ and, using (2-24), we see that

$$
\widetilde{\mathscr{T}}_{\varepsilon, N}^{N}=\frac{1}{2} \sum_{a, b=0}^{n} g^{a b} v_{y^{a}} v_{y^{b}}-\frac{1}{2}\left(v_{y^{N}}\right)^{2}+\frac{1}{\varepsilon^{2}} F(v)=-\frac{1}{2}\left(v_{y^{N}}\right)^{2}+\frac{1}{\varepsilon^{2}} F(v)+O\left(\left|D_{\tau} v\right|^{2}\right) .
$$

We will write

$$
\Theta_{2}\left(y^{\tau}\right):=\delta_{\varepsilon} \int_{B_{v}\left(\rho_{0}\right)}\left|v_{y^{N}}^{2}-\frac{1}{\varepsilon^{2}} F(v)\right| d y^{v},
$$

and we will now say that $y^{\tau} \in\left(-T_{1}, T_{1}\right) \times \mathbb{T}^{n}$ is good if

$$
\mathscr{D}_{v}\left(v\left(y^{\tau}\right)\right) \leq \kappa_{3} \quad \text { and, in addition, } \quad \Theta_{1}\left(y^{\tau}\right) \leq \kappa_{4}
$$

for $\kappa_{3}, \kappa_{4}$ found in Lemma 11. Then, Lemma 11 implies that, if $y^{\tau}$ is good, then

$$
\Theta_{2}\left(y^{\tau}\right) \leq C \sqrt{\left|\Theta_{1}\left(y^{\tau}\right)\right|+\zeta_{0}} .
$$

Thus, using Hölder's inequality

$$
\int_{\{\text {good points\} }} \Theta_{2}\left(y^{\tau}\right) d y^{\tau} \leq \sqrt{C \int_{\{\text {good points\} }}\left(\left|\Theta_{1}\left(y^{\tau}\right)\right|+C \zeta_{0}\right) d y^{\tau}} \leq C \sqrt{\zeta_{0}},
$$

using estimates from Case 3 above. And, if $y^{\tau}$ is bad, then

$$
\Theta_{2}\left(y^{\tau}\right) \leq C\left(1+\Theta_{1}\left(y^{\tau}\right)\right)
$$

so that

$$
\begin{aligned}
\int_{\{\text {bad points }\}} \Theta_{2}\left(y^{\tau}\right) d y^{\tau} & \leq \sqrt{C \int_{\{\text {bad points }\}}\left(\Theta_{1}\left(y^{\tau}\right)+1\right) d y^{\tau}} \\
& \leq C \sqrt{\zeta_{0}}+C\left(\mathscr{H}^{1+n}\left(\left\{y^{\tau} \in\left(-T_{0}, T_{0}\right) \times \mathbb{T}^{n}: y^{\tau} \text { is bad }\right\}\right)\right)^{1 / 2} \leq C \sqrt{\zeta_{0}},
\end{aligned}
$$

where at the end we used Chebyshev's inequality with (6-3) and (6-4). Hence,

$$
\left|\delta_{\varepsilon} \int_{\left(-T_{1}, T_{1}\right) \times \mathbb{T}^{n} \times B_{v}\left(\rho_{0}\right)} \check{m}_{N}^{N} \widetilde{\mathscr{T}}_{\varepsilon, N}^{N} d y\right| \leq C \int_{\left(-T_{0}, T_{0}\right) \times \mathbb{J}^{n}} \Theta_{2}\left(y^{\tau}\right) d y^{\tau}+O\left(\zeta_{0}\right) \leq C \sqrt{\zeta_{0}} .
$$

$\underline{k=2:}$ We claim that, when $k=2$,

$$
\left|\delta_{\varepsilon} \int_{\left(-T_{1}, T_{1}\right) \times \mathbb{T}^{n} \times B_{v}\left(\rho_{0}\right)} \check{m}_{\gamma}^{\delta} \widetilde{\mathscr{T}}_{\varepsilon, \delta}^{\gamma} d y\right| \leq C \sqrt{\zeta_{0}}
$$

if $\delta, \gamma \in\{N-1, N\}$. This will complete the proof of (6-5). To prove (6-27), we first note that Proposition 4 implies that

$$
\left(\begin{array}{cc}
\widetilde{\mathscr{T}}_{\varepsilon, N-1}^{N-1} & \widetilde{\mathscr{T}}_{\varepsilon, N}^{N-1} \\
\widetilde{\mathscr{T}}_{\varepsilon, N-1}^{N} & \widetilde{\mathscr{T}}_{\varepsilon, N}^{N}
\end{array}\right)=\left(\begin{array}{cc}
\frac{1}{2}\left(\left|v_{y^{N}}\right|^{2}-\left|v_{y^{N-1}}\right|^{2}\right)+\varepsilon^{-2} F(v) & -v_{y^{N-1}} \cdot v_{y^{N}} \\
-v_{y^{N-1}} \cdot v_{y^{N}} & \frac{1}{2}\left(-\left|v_{y^{N}}\right|^{2}+\left|v_{y^{N-1}}\right|^{2}\right)+\varepsilon^{-2} F(v)
\end{array}\right)
$$


At this point, we need [Kurzke and Spirn 2009, Theorem 1], which implies that, if $w \in H^{1}\left(B_{v}\left(\rho_{0}\right), \mathbb{R}^{2}\right)$,

$$
\mathscr{D}_{v}(w) \leq \kappa_{3} \quad \text { and } \quad \Theta_{1}\left(y^{\tau}\right) \leq \frac{3}{2}
$$

then

$$
\left|\delta_{\varepsilon} \int_{B_{\nu}\left(\rho_{0}\right)}\left(\begin{array}{cc}
\left|w_{y^{N-1}}\right|^{2} & w_{y^{N-1}} \cdot w_{y^{N}} \\
w_{y^{N-1}} \cdot v_{y^{N}} & \left|w_{y^{N}}\right|^{2}
\end{array}\right) d y^{v}-\left(\begin{array}{cc}
1 & 0 \\
0 & 1
\end{array}\right)\right| \leq C\left(\delta_{\varepsilon} \int_{B_{\nu}\left(\rho_{0}\right)} e_{\varepsilon, v}(w) d y^{\nu}-1+C \delta_{\varepsilon}\right)^{1 / 2} .
$$

(The main hypothesis of the Kurzke-Spirn estimate is (5-9), and we have shown in the proof of Proposition 17 that this follows from (6-29).) Accordingly, we will continue to say (exactly parallel to the case $k=1$; see (6-26)) that $y^{\tau} \in\left(-T_{1}, T_{1}\right) \times \mathbb{T}^{n}$ is $\operatorname{good}$ if $v\left(y^{\tau}, \cdot\right) \in H^{1}\left(B_{v}\left(\rho_{0}\right) ; \mathbb{R}^{2}\right)$ satisfies (6-29). A point that is not good is said to be bad. It follows, as usual, from Chebyshev's inequality and (6-3), (6-4) that

$$
\mathscr{H}^{1+n}\left(\left\{y^{\tau} \in\left(-T_{0}, T_{0}\right) \times \mathbb{T}^{n}: y^{\tau} \text { is bad }\right\}\right) \leq C \zeta_{0} .
$$

The Kurzke-Spirn inequality implies that, if $y^{\tau}$ is good, then

$$
\frac{\delta_{\varepsilon}}{2} \int_{B_{\nu}(\rho)}\left|\nabla_{\nu} v\left(y^{\tau}, y^{\nu}\right)\right|^{2} d y^{\nu} \geq 1-C\left(\Theta_{1}\left(y^{\tau}\right)+C \delta_{\varepsilon}\right)^{1 / 2} .
$$

Thus, for a good point $y^{\tau}$,

$$
\begin{aligned}
\delta_{\varepsilon} \int_{B_{v}\left(\rho_{0}\right)} \frac{1}{\varepsilon^{2}} F(v)\left(y^{\tau}, y^{\nu}\right) d y^{v} & =\Theta_{1}\left(y^{\tau}\right)+\left(1-\frac{\delta_{\varepsilon}}{2} \int_{B_{v}(\rho)}\left|\nabla_{\nu} v\left(y^{\tau}, y^{\nu}\right)\right|^{2} d y^{v}\right) \\
& \leq \Theta_{1}\left(y^{\tau}\right)+C\left(\Theta_{1}\left(y^{\tau}\right)+C \delta_{\varepsilon}\right)^{1 / 2} .
\end{aligned}
$$

Similarly, the Kurzke-Spirn estimate also implies that, if $y^{\tau}$ is good, then

$$
\delta_{\varepsilon}\left|\int_{B_{v}\left(\rho_{0}\right)}\left(\left|v_{y^{N}}\right|^{2}-\left|v_{y^{N-1}}\right|^{2}\right) d y^{v}\right|+\delta_{\varepsilon}\left|\int_{B_{v}\left(\rho_{0}\right)} v_{y^{N}} \cdot v_{y^{N-1}} d y^{v}\right| \leq C\left(\Theta_{1}\left(y^{\tau}\right)+C \delta_{\varepsilon}\right)^{1 / 2} .
$$

Combining these and recalling (6-28), we see that, if $y^{\tau}$ is good, then, for $\gamma, \delta \in\{N-1, N\}$,

$$
\left|\delta_{\varepsilon} \int_{B_{v}\left(\rho_{0}\right)} \mathscr{T}_{\varepsilon, \gamma}^{\delta}\left(y^{\tau}, y^{v}\right) d y^{v}\right| \leq C\left(\Theta_{1}\left(y^{\tau}\right)+C \delta_{\varepsilon}\right)^{1 / 2}+\Theta_{3}\left(y^{\tau}\right),
$$

where $\Theta_{3}\left(y^{\tau}\right):=\delta_{\varepsilon} \int_{B_{v}\left(\rho_{0}\right)}\left(\left|D_{\tau} v\right|^{2}+\left|y^{\nu}\right|^{2}\left|\nabla_{v} v\right|^{2}\right) d y^{\nu}$. Also, if $y^{\tau}$ is bad, then (6-28) implies that

$$
\delta_{\varepsilon} \int_{B_{v}\left(\rho_{0}\right)}\left|\mathscr{T}_{\varepsilon, \gamma}^{\delta}\left(y^{\tau}, y^{\nu}\right)\right| d y^{\nu} \leq C\left(\Theta_{1}\left(y^{\tau}\right)+1\right) .
$$

This last fact, together with the estimate of the bad set's size and (6-3), implies that

$$
\left|\delta_{\varepsilon} \int_{\left(-T_{1}, T_{1}\right) \times \mathbb{T}^{n} \times B_{v}\left(\rho_{0}\right)} \check{m}_{\gamma}^{\delta} \tilde{\widetilde{T}}_{\varepsilon, \delta}^{\gamma} d y\right| \leq|A|+|B|+C \zeta_{0}
$$


where

$$
\begin{aligned}
& A:=\delta_{\varepsilon} \int_{\left\{\text {good points } \in\left(-T_{1}, T_{1}\right) \times \mathbb{\mathbb { T }}^{n}\right\}} \int_{B_{\nu}(\rho)} \check{m}_{\gamma}^{\delta}\left(y^{\tau}, 0\right) \tilde{\mathscr{T}}_{\varepsilon, \delta}^{\gamma} d y^{\nu} d y^{\tau}, \\
& B:=\delta_{\varepsilon} \int_{\left\{\text {good points } \in\left(-T_{1}, T_{1}\right) \times \mathbb{T}^{n}\right\}} \int_{B_{\gamma}(\rho)}\left(\check{m}_{\gamma}^{\delta}\left(y^{\tau}, y^{v}\right)-\check{m}_{\gamma}^{\delta}\left(y^{\tau}, 0\right)\right) \tilde{\mathscr{T}}_{\varepsilon, \delta}^{\gamma} d y^{\nu} d y^{\tau} .
\end{aligned}
$$

From (6-30), Hölder's inequality, (6-2), and $(6-3)$, we see that

$$
|A| \leq\|\check{m}\|_{\infty} \int_{\left\{\text {good points } \in\left(-T_{1}, T_{1}\right) \times \mathbb{T}^{n}\right\}} C\left(\left(\Theta_{1}\left(y^{\tau}\right)+C \delta_{\varepsilon}\right)^{1 / 2}+\Theta_{3}\left(y^{\tau}\right)\right) d y^{\tau} \leq C \sqrt{\zeta_{0}} .
$$

And, since $\|\hat{m}\|_{W^{1, \infty}} \leq C$,

$$
|B| \leq C \delta_{\varepsilon} \int_{\left(-T_{1}, T_{1}\right) \times \mathbb{T}^{n} \times B_{v}\left(\rho_{0}\right)}\left|y^{v}\right| e_{\varepsilon, v}(v) d y .
$$

We have shown, in Case 3 above, that the right-hand side is bounded by $C \sqrt{\zeta_{0}}$, so we find that $|A|+|B| \leq$ $C \sqrt{\zeta_{0}}$. Therefore, we have proved (6-27), and (6-5) follows.

\section{Appendix}

In this appendix, we give the proof of Propositions 4 and 5. Recall that we have defined $G=D \psi^{T} \eta D \psi$, where $\psi\left(y^{\tau}, y^{v}\right):=H\left(y^{\tau}\right)+\sum_{i=1}^{k} \bar{v}_{i}\left(y^{\tau}\right) y^{n+i}$. Here, $H$ is the given parametrization of the minimal surface $\Gamma$, and the vectors $\left\{\bar{v}_{i}\right\}$ form an orthonormal frame for the normal bundle of $\Gamma$; see (2-11). The proposition asserts certain properties of $g:=\operatorname{det} G$ and $\left(g^{i j}\right)=G^{-1}$. We will use the following lemma to read off properties of $G^{-1}$ from those of $G$.

Lemma 26. Let $M$ be a matrix written in block form as

$$
M=\left(\begin{array}{ll}
A & B \\
C & D
\end{array}\right)
$$

(where the blocks need not be of equal size, i.e., $A \in M^{n \times n}, B \in M^{n \times m}, C \in M^{m \times n}$ and $D \in M^{m \times m}$, for some $m$ and $n$ ). If

$$
N=\left(\begin{array}{cc}
\left(A-B D^{-1} C\right)^{-1} & -A^{-1} B\left(D-C A^{-1} B\right)^{-1} \\
-D^{-1} C\left(A-B D^{-1} C\right)^{-1} & \left(D-C A^{-1} B\right)^{-1}
\end{array}\right)
$$

is well defined, then $N=M^{-1}$.

This is proved by simply verifying that $M N=I$.

Proof of Propositions 4 and 5. We will think of $v\left(y^{\tau}\right)$ as a $(1+N) \times k$ matrix, with columns $\bar{v}_{i}$ for $i=1, \ldots, k$, and of $y^{v}$ as a $k \times 1$ vector, so that $v y^{v}:=\sum_{i=1}^{k} \bar{v}_{i}\left(y^{\tau}\right) y^{n+i}$.

Step 1: To start, note that $\nabla_{\nu} \psi(y)=v\left(y^{\tau}\right)$, so the choice (2-11) of $v$ implies that $G$ can be written in block form as

where

$$
G=\left(\begin{array}{cc}
G_{\tau \tau} & G_{\tau \nu} \\
G_{v \tau} & I_{k}
\end{array}\right)
$$

$$
G_{\tau \tau}:=D_{\tau} \psi^{T} \eta D_{\tau} \psi \in M^{(1+n) \times(1+n)}, \quad G_{\tau \nu}=G_{\nu \tau}^{T}:=D_{\tau}\left(\nu y^{\nu}\right)^{T} \eta \nu \in M^{(1+n) \times k}
$$


and $I_{k}$ denotes the $k \times k$ identity matrix. Observe that

$$
\left|G_{\tau \nu}\right| \leq C\left|y^{\nu}\right| \text { when } k \geq 2 \text { and } G_{\tau \nu} \equiv 0 \text { for } k=1 .
$$

The second assertion above follows from differentiating the identity $\left(v^{T} \eta v\right)\left(y^{\tau}\right) \equiv 1$. Since $\psi\left(y^{\tau}, 0\right)=$ $H\left(y^{\tau}\right)$, we can write $G_{\tau \tau}\left(y^{\tau}, 0\right)$ in block form as

$$
G_{\tau \tau}\left(y^{\tau}, 0\right)=\left(\begin{array}{cc}
H_{y_{0}}^{T} \eta H_{y_{0}} & H_{y_{0}}^{T} \eta \nabla H \\
\nabla H^{T} \eta H_{y_{0}} & \nabla H^{T} \eta \nabla H
\end{array}\right)=\left(\begin{array}{cc}
-1+\left|h_{y^{0}}\right|^{2} & 0 \\
0 & \nabla h^{T} \nabla h
\end{array}\right),
$$

where we have used (2-5) and (2-6). It then follows, from (2-7) and the smoothness of $H$, that $G_{\tau \tau}\left(y^{\tau}, 0\right)$ is invertible, with uniformly bounded inverse, for $y^{\tau} \in\left[-T_{1}, T_{1}\right] \times \mathbb{T}^{n}$. If $\rho_{0}$ is chosen small enough, it follows by continuity that $G_{\tau \tau}(y)$ is invertible, with uniformly bounded inverse, for $y \in\left[-T_{1}, T_{1}\right] \times$ $\mathbb{T}^{n} \times B_{v}\left(\rho_{0}\right)$.

Step 2: Next, we note that $G_{\tau \tau}(y)=G_{\tau \tau}\left(y^{\tau}, 0\right)+O\left(\left|y^{\tau}\right|\right)$; we use (-31) and (-32) to find that, taking $\rho_{0}$ smaller if necessary, $G(y)$ is invertible for $y \in\left[-T_{1}, T_{1}\right] \times \mathbb{T}^{n} \times B_{v}\left(\rho_{0}\right)$, with

$$
\begin{aligned}
G^{-1}(y) & =\left(\begin{array}{cc}
\left(G_{\tau \tau}\left(y^{\tau}, 0\right)+O\left(\left|y^{\nu}\right|\right)\right)^{-1} & O\left(\left|y^{\nu}\right|\right) \\
O\left(\left|y^{\nu}\right|\right) & \left(I_{k}-O\left(\left|y^{\nu}\right|^{2}\right)\right)^{-1}
\end{array}\right) \\
& =\left(\begin{array}{cc}
G_{\tau \tau}^{-1}\left(y^{\tau}, 0\right) & 0 \\
0 & I_{k}
\end{array}\right)+\left(\begin{array}{cc}
O\left(\left|y^{\nu}\right|\right) & O\left(\left|y^{\nu}\right|\right) \\
O\left(\left|y^{\nu}\right|\right) & O\left(\left|y^{\nu}\right|^{2}\right)
\end{array}\right) .
\end{aligned}
$$

We have used (more than once) the fact that $G_{\tau \tau}^{-1}\left(y^{\tau}, 0\right)$ is uniformly bounded, which implies that $\mid\left(G_{\tau \tau}+\right.$ $A)^{-1}-G_{\tau \tau}^{-1}|\leq C| A \mid$ for $A$ sufficiently small, with a uniform constant $C$.

From (-33) and (2-7), we easily conclude that (2-20), (2-19), and the first estimate of (2-17) hold. Moreover, if $k=1$, then, in view of $(-32)$,

$$
G^{-1}(y)=\left(\begin{array}{cc}
G_{\tau \tau}(y)^{-1} & 0 \\
0 & I_{k}
\end{array}\right)=G^{-1}(y)=\left(\begin{array}{cc}
G_{\tau \tau}\left(y^{\tau}, 0\right)^{-1}+O\left(\left|y^{v}\right|\right) & 0 \\
0 & I_{k}
\end{array}\right),
$$

from which we infer (2-24) and (2-25).

To establish the second conclusion of (2-17), we differentiate the identity $G^{-1} G=I$ to find that

$$
G_{y_{0}}^{-1}=-G^{-1} G_{y_{0}} G^{-1} .
$$

Our earlier expression for $G$ implies that

$$
G_{y_{0}}=\left(\begin{array}{cc}
G_{\tau \tau, y_{0}} & O\left(\left|y^{\nu}\right|\right) \\
O\left(\left|y^{\nu}\right|\right) & 0
\end{array}\right)
$$

and one can readily check that this implies that $\left|g_{y^{0}}^{\alpha \beta} \xi_{\alpha} \xi_{\beta}\right| \leq C\left(\left|\xi_{\tau}\right|^{2}+\left|y^{\nu}\right|^{2}\left|\xi_{v}\right|^{2}\right)$, which completes the proof of (2-17).

Step 3: It remains to establish (2-18). To do this, fix $\zeta \in C_{0}^{\infty}\left(\left[-T_{1}, T_{1}\right] \times \mathbb{T}^{n} ; \mathbb{R}^{k}\right)$ and, for $\sigma \in \mathbb{R}$, define

where

$$
f(\sigma)=\int_{V}\left(-\operatorname{det}\left(D_{\tau} H_{\sigma}^{T} \eta D H_{\sigma}\right)\right)^{1 / 2} d y^{\tau},
$$

$$
H_{\sigma}\left(y^{\tau}\right)=H\left(y^{\tau}\right)+\sigma v\left(y^{\tau}\right) \zeta\left(y^{\tau}\right)=\psi\left(y^{\tau}, \sigma \zeta\left(y^{\tau}\right)\right) \text {. }
$$


Note that, for $\sigma$ small, $H_{\sigma}$ parametrizes a surface $\Gamma_{\sigma}$ that is a small variation of the original surface $\Gamma$. Because $\Gamma$ is a Minkowski minimal surface, it follows that $f^{\prime}(0)=0$. We will show that this yields the conclusion of the lemma.

Thinking of $D \zeta$ as a $k \times(1+n)$ matrix, a direct computation yields

$$
D H_{\sigma}\left(y^{\tau}\right)=D_{\tau} \psi\left(y^{\tau}, \sigma \zeta\left(y^{\tau}\right)\right)+\sigma v\left(y^{\tau}\right) D \zeta\left(y^{\tau}\right)
$$

It then follows from (2-11) that $D H_{\sigma}^{T} \eta D H_{\sigma}$ has the form

$$
\left(D H_{\sigma}^{T} \eta D H_{\sigma}\right)\left(y^{\tau}\right)=\left(D_{\tau} \psi^{T} \eta D_{\tau} \psi\right)\left(y^{\tau}, \sigma \zeta\left(y^{\tau}\right)\right)+\sigma^{2} B\left(y^{\tau}\right) .
$$

for some matrix $B\left(y^{\tau}\right)$ that depends smoothly on $y^{\tau}$. Since, if $A(\sigma)$ are square matrices depending smoothly on a real parameter $\sigma$, then

it follows that, at $\sigma=0$,

$$
\left.\frac{d}{d \sigma} \operatorname{det}\left(A(\sigma)+\sigma^{2} B\right)\right|_{\sigma=0}=\left.\frac{d}{d \sigma} \operatorname{det} A(\sigma)\right|_{\sigma=0},
$$

$$
\frac{d}{d \sigma} \operatorname{det}\left(D H_{\sigma}^{T} \eta D H_{\sigma}\right)\left(y^{\tau}\right)=\frac{d}{d \sigma} \operatorname{det}\left(D_{\tau} \psi^{T} \eta D_{\tau} \psi\right)\left(y^{\tau}, \sigma \zeta\left(y^{\tau}\right)\right)
$$

Step 4: We next note that

$$
\operatorname{det}\left(D_{\tau} \psi^{T} \eta D_{\tau} \psi\right)\left(y^{\tau}, \sigma \zeta\left(y^{\tau}\right)\right)=\operatorname{det}\left(D \psi^{T} \eta D \psi\right)\left(y^{\tau}, \sigma \zeta\left(y^{\tau}\right)\right)+O\left(\sigma^{2}\right) .
$$

Indeed, this follows (by rather easy linear algebra considerations) from the fact that

$$
D \psi^{T} \eta D \psi\left(y^{\tau}, \sigma \zeta\left(y^{\tau}\right)\right)=\left(\begin{array}{cc}
D_{\tau} \psi^{T} \eta D_{\tau} \psi & O(\sigma) \\
O(\sigma) & I_{k}+O\left(\sigma^{2}\right)
\end{array}\right) .
$$

By combining (-35) and (-36),

$$
\left.\frac{d}{d \sigma} \operatorname{det}\left(D H_{\sigma}^{T} \eta D H_{\sigma}\right)\left(y^{\tau}\right)\right|_{\sigma=0}=\left.\frac{d}{d \sigma} g\left(y^{\tau}, \sigma \zeta\left(y^{\tau}\right)\right)\right|_{\sigma=0}=\nabla_{\nu} g\left(y^{\tau}, 0\right) \cdot \zeta
$$

at $\sigma=0$. Also, it follows from (2-7) and continuity that $\operatorname{det}\left(D H_{\sigma}^{T} \eta D H_{\sigma}\right)\left(y^{\tau}\right)$ and $g\left(y^{\tau}, \sigma \zeta\left(y^{\tau}\right)\right)$ are bounded away from 0 for $\zeta$ small enough, and hence

$$
\left.\frac{d}{d \sigma}\left(-\operatorname{det}\left(D H_{\sigma}^{T} \eta D H_{\sigma}\right)\left(y^{\tau}\right)\right)^{1 / 2}\right|_{\sigma=0}=\nabla_{\nu} \sqrt{-g} \cdot \zeta
$$

Thus, the identity $f^{\prime}(0)=0$ reduces to

$$
0=\int \nabla_{\nu} \sqrt{-g} \cdot \zeta d y^{\tau}
$$

Since $\zeta$ is arbitrary, we conclude that $\nabla_{\nu} \sqrt{-g}=0$. This and (-33) imply the required estimate (2-18).

\section{Acknowledgments}

The author is very grateful to Alberto Montero for numerous useful discussions, including conversations which provided the initial impetus for this project, and for carefully reading and commenting on drafts of large parts of this paper. 


\section{References}

[Alberti et al. 2003] G. Alberti, S. Baldo, and G. Orlandi, "Functions with prescribed singularities", J. Eur. Math. Soc. (JEMS) 5:3 (2003), 275-311. MR 2004g:49068 Zbl 1033.46028

[Alberti et al. 2005] G. Alberti, S. Baldo, and G. Orlandi, "Variational convergence for functionals of Ginzburg-Landau type", Indiana Univ. Math. J. 54:5 (2005), 1411-1472. MR 2007a:49017 Zbl 1160.35013

[Ambrosio and Soner 1997] L. Ambrosio and H. M. Soner, "A measure-theoretic approach to higher codimension mean curvature flows", Ann. Scuola Norm. Sup. Pisa Cl. Sci. (4) 25:1-2 (1997), 27-49. MR 99m:35217 Zbl 1043.35136

[Barles et al. 1993] G. Barles, H. M. Soner, and P. E. Souganidis, "Front propagation and phase field theory", SIAM J. Control Optim. 31:2 (1993), 439-469. MR 94c:35005 Zbl 0785.35049

[Bellettini et al. 2008] G. Bellettini, M. Novaga, and M. Orlandi, "Time-like lorentzian minimal submanifolds as singular limits of nonlinear wave equations", preprint, 2008. arXiv 0811.3741

[Bethuel et al. 2001] F. Bethuel, H. Brezis, and G. Orlandi, "Asymptotics for the Ginzburg-Landau equation in arbitrary dimensions", J. Funct. Anal. 186:2 (2001), 432-520. MR 2002k:35081a Zbl 1077.35047

[Bethuel et al. 2006] F. Bethuel, G. Orlandi, and D. Smets, "Convergence of the parabolic Ginzburg-Landau equation to motion by mean curvature”, Ann. of Math. (2) 163:1 (2006), 37-163. MR 2007b:53135 Zbl 1103.35038

[Brendle 2002] S. Brendle, "Hypersurfaces in Minkowski space with vanishing mean curvature", Comm. Pure Appl. Math. 55:10 (2002), 1249-1279. MR 2003j:58043 Zbl 1042.53041

[Bronsard and Kohn 1991] L. Bronsard and R. V. Kohn, "Motion by mean curvature as the singular limit of Ginzburg-Landau dynamics", J. Differential Equations 90:2 (1991), 211-237. MR 92d:35037 Zbl 0735.35072

[Chen 1992] X. Chen, "Generation and propagation of interfaces for reaction-diffusion equations", J. Differential Equations 96:1 (1992), 116-141. MR 92m:35129 Zbl 0765.35024

[Cuccagna 2008] S. Cuccagna, "On asymptotic stability in 3D of kinks for the $\varphi^{4}$ model", Trans. Amer. Math. Soc. 360:5 (2008), 2581-2614. MR 2009e:35181 Zbl 1138.35062

[Evans et al. 1992] L. C. Evans, H. M. Soner, and P. E. Souganidis, "Phase transitions and generalized motion by mean curvature", Comm. Pure Appl. Math. 45:9 (1992), 1097-1123. MR 93g:35064 Zbl 0801.35045

[Gustafson and Sigal 2006] S. Gustafson and I. M. Sigal, "Effective dynamics of magnetic vortices", Adv. Math. 199:2 (2006), 448-498. MR 2007d:82108 Zbl 1081.35102

[Hutchinson and Tonegawa 2000] J. E. Hutchinson and Y. Tonegawa, "Convergence of phase interfaces in the van der WaalsCahn-Hilliard theory", Calc. Var. Partial Differential Equations 10:1 (2000), 49-84. MR 2001m:49070 Zbl 1070.49026

[Ilmanen 1993] T. Ilmanen, "Convergence of the Allen-Cahn equation to Brakke's motion by mean curvature", J. Differential Geom. 38:2 (1993), 417-461. MR 94h:58051 Zbl 0784.53035

[Jerrard 1999] R. L. Jerrard, "Vortex dynamics for the Ginzburg-Landau wave equation", Calc. Var. Partial Differential Equations 9:1 (1999), 1-30. MR 2000i:35189 Zbl 0941.35099

[Jerrard 2007] R. L. Jerrard, "Local minimizers with vortex filaments for a Gross-Pitaevsky functional”, ESAIM Control Optim. Calc. Var. 13:1 (2007), 35-71. MR 2008g:58026 Zbl 1111.35077

[Jerrard and Soner 1999] R. L. Jerrard and H. M. Soner, "Scaling limits and regularity results for a class of Ginzburg-Landau systems", Ann. Inst. H. Poincaré Anal. Non Linéaire 16:4 (1999), 423-466. MR 2000f:35135 Zbl 0944.35006

[Jerrard and Spirn 2007] R. Jerrard and D. Spirn, "Refined Jacobian estimates for Ginzburg-Landau functionals", Indiana Univ. Math. J. 56:1 (2007), 135-186. MR 2008h:35043 Zbl 1121.26008

[Kibble 1976] T. Kibble, “Topology cosmic domains and strings”, J. Physics A 9 (1976), 1387-1398. Zbl 0333.57005

[Kurzke and Spirn 2009] M. Kurzke and D. Spirn, "Quantitative equipartition of the Ginzburg-Landau functional with applications", preprint 442, SBF 611, Universität Bonn, 2009, available at sfb611.iam.uni-bonn.de/uploads/442-komplett.pdf. To appear in Indiana Math. J.

[Lin 1998] F. H. Lin, "Complex Ginzburg-Landau equations and dynamics of vortices, filaments, and codimension-2 submanifolds”, Comm. Pure Appl. Math. 51:4 (1998), 385-441. MR 98k:35177 Zbl 0932.35121 
[Lin 1999] F. H. Lin, "Vortex dynamics for the nonlinear wave equation", Comm. Pure Appl. Math. 52:6 (1999), 737-761. MR 99m:35166 Zbl 0929.35076

[Lin and Rivière 1999] F. Lin and T. Rivière, "Complex Ginzburg-Landau equations in high dimensions and codimension two area minimizing currents”, J. Eur. Math. Soc. (JEMS) 1:3 (1999), 237-311. MR 2000g:49048 Zbl 0939.35056

[Lin and Rivière 2001] F.-H. Lin and T. Rivière, "A quantization property for moving line vortices", Comm. Pure Appl. Math. 54:7 (2001), 826-850. MR 2002c:35140 Zbl 1029.35127

[Lindblad 2004] H. Lindblad, "A remark on global existence for small initial data of the minimal surface equation in Minkowskian space time”, Proc. Amer. Math. Soc. 132:4 (2004), 1095-1102. MR 2005a:35203 Zbl 1061.35053

[Milbredt 2008] O. Milbredt, The Cauchy problem for membranes, dissertation, Freie Universität Berlin, 2008.

[Modica 1987] L. Modica, "The gradient theory of phase transitions and the minimal interface criterion", Arch. Rational Mech. Anal. 98:2 (1987), 123-142. MR 88f:76038 Zbl 0616.76004

[de Mottoni and Schatzman 1995] P. de Mottoni and M. Schatzman, "Geometrical evolution of developed interfaces", Trans. Amer. Math. Soc. 347:5 (1995), 1533-1589. MR 2000a:35022 Zbl 0840.35010

[Neu 1990] J. C. Neu, "Kinks and the minimal surface equation in Minkowski space", Phys. D 43:2-3 (1990), 421-434. MR 91i:35190 Zbl 0704.58051

[Pacard and Ritoré 2003] F. Pacard and M. Ritoré, "From constant mean curvature hypersurfaces to the gradient theory of phase transitions", J. Differential Geom. 64:3 (2003), 359-423. MR 2005e:58018 Zbl 1070.58014

[Rotstein and Nepomnyashchy 2000] H. G. Rotstein and A. A. Nepomnyashchy, "Dynamics of kinks in two-dimensional hyperbolic models", Phys. D 136:3-4 (2000), 245-265. MR 2000k:35201 Zbl 0946.35050

[Sandier and Serfaty 2007] E. Sandier and S. Serfaty, Vortices in the magnetic Ginzburg-Landau model, Progress in Nonlinear Differential Equations and their Applications 70, Birkhäuser, Boston, MA, 2007. MR 2008g:82149 Zbl 1112.35002

[Shatah 1988] J. Shatah, "Weak solutions and development of singularities of the SU(2) $\sigma$-model", Comm. Pure Appl. Math. 41:4 (1988), 459-469. MR 89f:58044 Zbl 0686.35081

[Shatah and Struwe 1998] J. Shatah and M. Struwe, Geometric wave equations, Courant Lecture Notes in Mathematics 2, New York University Courant Institute of Mathematical Sciences, New York, 1998. MR 2000i:35135 Zbl 0993.35001

[Soner 1998] H. M. Soner, "Front propagation", pp. 185-206 in Boundaries, interfaces, and transitions (Banff, AB, 1995), edited by M. C. Delfour, CRM Proc. Lecture Notes 13, Amer. Math. Soc., Providence, RI, 1998. MR 2000e:35104 Zbl 0914. 35065

[Stuart 2004a] D. M. A. Stuart, "The geodesic hypothesis and non-topological solitons on pseudo-Riemannian manifolds", Ann. Sci. École Norm. Sup. (4) 37:2 (2004), 312-362. MR 2005b:58040 Zbl 1054.58026

[Stuart 2004b] D. M. A. Stuart, "Geodesics and the Einstein nonlinear wave system”, J. Math. Pures Appl. (9) 83:5 (2004), 541-587. MR 2005b:58041 Zbl 1072.58021

[Vilenkin and Shellard 1994] A. Vilenkin and E. P. S. Shellard, Cosmic strings and other topological defects, Cambridge Monographs on Mathematical Physics, Cambridge University Press, 1994. MR 98a:83134 Zbl 0978.83052

Received 1 Dec 2009. Accepted 15 Apr 2010.

ROBERT JERRARD: rjerrard@math.toronto.edu

University of Toronto, Toronto M4X 1S9, Canada 


\section{Analysis \& PDE}

pjm.math.berkeley.edu/apde

EDITORS

EDITOR-IN-CHIEF

Maciej Zworski

University of California

Berkeley, USA

BOARD OF EDITORS

\begin{tabular}{|c|c|c|c|}
\hline Michael Aizenman & $\begin{array}{l}\text { Princeton University, USA } \\
\text { aizenman@math.princeton.edu }\end{array}$ & Nicolas Burq & $\begin{array}{l}\text { Université Paris-Sud 11, France } \\
\text { nicolas.burq@math.u-psud.fr }\end{array}$ \\
\hline Luis A. Caffarelli & $\begin{array}{l}\text { University of Texas, USA } \\
\text { caffarel@ math.utexas.edu }\end{array}$ & un-Yung Alice Chang & $\begin{array}{l}\text { Princeton University, USA } \\
\text { chang@math.princeton.edu }\end{array}$ \\
\hline Michael Christ & $\begin{array}{l}\text { University of California, Berkeley, USA } \\
\text { mchrist@math.berkeley.edu }\end{array}$ & Charles Fefferman & $\begin{array}{l}\text { Princeton University, USA } \\
\text { cf@math.princeton.edu }\end{array}$ \\
\hline Ursula Hamenstaedt & $\begin{array}{l}\text { Universität Bonn, Germany } \\
\text { ursula@math.uni-bonn.de }\end{array}$ & Nigel Higson & $\begin{array}{l}\text { Pennsylvania State Univesity, USA } \\
\text { higson@math.psu.edu }\end{array}$ \\
\hline Vaughan Jones & $\begin{array}{l}\text { University of California, Berkeley, USA } \\
\text { vfr@math.berkeley.edu }\end{array}$ & Herbert Koch & $\begin{array}{l}\text { Universität Bonn, Germany } \\
\text { koch@math.uni-bonn.de }\end{array}$ \\
\hline Izabella Laba & $\begin{array}{l}\text { University of British Columbia, Canada } \\
\text { ilaba@math.ubc.ca }\end{array}$ & Gilles Lebeau & $\begin{array}{l}\text { Université de Nice Sophia Antipolis, France } \\
\text { lebeau@unice.fr }\end{array}$ \\
\hline László Lempert & $\begin{array}{l}\text { Purdue University, USA } \\
\text { lempert@math.purdue.edu }\end{array}$ & Richard B. Melrose & $\begin{array}{l}\text { Massachussets Institute of Technology, USA } \\
\text { rbm@math.mit.edu }\end{array}$ \\
\hline Frank Merle & $\begin{array}{l}\text { Université de Cergy-Pontoise, France } \\
\text { Frank.Merle@u-cergy.fr }\end{array}$ & William Minicozzi II & $\begin{array}{l}\text { Johns Hopkins University, USA } \\
\text { minicozz@ math.jhu.edu }\end{array}$ \\
\hline Werner Müller & $\begin{array}{l}\text { Universität Bonn, Germany } \\
\text { mueller@math.uni-bonn.de }\end{array}$ & Yuval Peres & $\begin{array}{l}\text { University of California, Berkeley, USA } \\
\text { peres@stat.berkeley.edu }\end{array}$ \\
\hline Gilles Pisier & $\begin{array}{l}\text { Texas A\&M University, and Paris } 6 \\
\text { pisier@math.tamu.edu }\end{array}$ & Tristan Rivière & $\begin{array}{l}\text { ETH, Switzerland } \\
\text { riviere@ math.ethz.ch }\end{array}$ \\
\hline Igor Rodnianski & $\begin{array}{l}\text { Princeton University, USA } \\
\text { irod@math.princeton.edu }\end{array}$ & Wilhelm Schlag & $\begin{array}{l}\text { University of Chicago, USA } \\
\text { schlag@math.uchicago.edu }\end{array}$ \\
\hline Sylvia Serfaty & $\begin{array}{l}\text { New York University, USA } \\
\text { serfaty@ cims.nyu.edu }\end{array}$ & Yum-Tong Siu & $\begin{array}{l}\text { Harvard University, USA } \\
\text { siu@math.harvard.edu }\end{array}$ \\
\hline Terence Tao & $\begin{array}{l}\text { University of California, Los Angeles, USA } \\
\text { tao@math.ucla.edu }\end{array}$ & Michael E. Taylor & $\begin{array}{l}\text { Univ. of North Carolina, Chapel Hill, USA } \\
\text { met@math.unc.edu }\end{array}$ \\
\hline Gunther Uhlmann & $\begin{array}{l}\text { University of Washington, USA } \\
\text { gunther@math.washington.edu }\end{array}$ & András Vasy & $\begin{array}{l}\text { Stanford University, USA } \\
\text { andras@math.stanford.edu }\end{array}$ \\
\hline an Virgil Voiculescu & $\begin{array}{l}\text { University of California, Berkeley, USA } \\
\text { dvv@ math.berkeley.edu }\end{array}$ & Steven Zelditch & $\begin{array}{l}\text { Northwestern University, USA } \\
\text { zelditch@math.northwestern.edu }\end{array}$ \\
\hline
\end{tabular}

PRODUCTION

contact@msp.org

Silvio Levy, Scientific Editor

Sheila Newbery, Senior Production Editor

See inside back cover or pjm.math.berkeley.edu/apde for submission instructions.

The subscription price for 2011 is US \$120/year for the electronic version, and \$180/year for print and electronic. Subscriptions, requests for back issues from the last three years and changes of subscribers address should be sent to Mathematical Sciences Publishers, Department of Mathematics, University of California, Berkeley, CA 94720-3840, USA.

Analysis \& PDE, at Mathematical Sciences Publishers, Department of Mathematics, University of California, Berkeley, CA 94720-3840 is published continuously online. Periodical rate postage paid at Berkeley, CA 94704, and additional mailing offices.

APDE peer review and production are managed by EditFLOW ${ }^{\mathrm{TM}}$ from Mathematical Sciences Publishers.

PUBLISHED BY

mathematical sciences publishers

http://msp.org/

A NON-PROFIT CORPORATION

Typeset in IATEX

Copyright $(2011$ by Mathematical Sciences Publishers 


\section{ANALYSIS \& PDE}

\section{Volume $4 \quad$ No. $2 \quad 2011$}

On the area of the symmetry orbits of cosmological spacetimes with toroidal or hyperbolic 191 symmetry

JACQUES SMULEVICI

On a maximum principle and its application to the logarithmically critical Boussinesq system 247 TAOUFIK HMIDI

Defects in semilinear wave equations and timelike minimal surfaces in Minkowski space

ROBERT JERRARD

Well- and ill-posedness issues for energy supercritical waves

Slim Ibrahim, Mohamed Majdoub and NADER Masmoudi 